\title{
On homotopy groups of the suspended classifying spaces
}

\author{
ROMAN MiKHAILOV \\ JIE WU
}

\begin{abstract}
In this paper, we determine the homotopy groups $\pi_{4}(\Sigma K(A, 1))$ and $\pi_{5}(\Sigma K(A, 1))$ for abelian groups $A$ by using the following methods from group theory and homotopy theory: derived functors, the Carlsson simplicial construction, the Baues-Goerss spectral sequence, homotopy decompositions and the methods of algebraic $K$-theory. As the applications, we also determine $\pi_{i}(\Sigma K(G, 1))$ with $i=4,5$ for some nonabelian groups $G=\Sigma_{3}$ and $\operatorname{SL}(\mathbb{Z})$, and $\pi_{4}\left(\Sigma K\left(A_{4}, 1\right)\right)$ for the 4-th alternating group $A_{4}$.
\end{abstract}

55Q52; 55P20, 55P40, 55P65, 55Q35

\section{Introduction}

It is well-known that the suspension functor applied to a topological space shifts homology groups, but "chaotically" changes homotopy groups. For example, one can take a circle $S^{1}$, whose homotopy type is very simple. Its suspension $\Sigma S^{1}=S^{2}$ has obvious homology groups, however the problem of investigating the homotopy groups of $S^{2}$ is one of the deepest problems of algebraic topology. Consider the following functors from the category of groups to the category of abelian groups:

$$
\pi_{n}\left(\Sigma^{m} K(-, 1)\right): \mathrm{Gr} \rightarrow \mathrm{Ab}, \quad n \geq 1, m \geq 1
$$

defined by $A \mapsto \pi_{n}\left(\Sigma^{m} K(A, 1)\right)$, where $\Sigma^{m}$ is the $m$-fold suspension. It is clear that $\pi_{n}\left(\Sigma^{m} K(\mathbb{Z}, 1)\right)=\pi_{n}\left(S^{m+1}\right)$, that is the homotopy groups of spheres appear as the simplest case of a general theory of homotopy groups of suspensions of classifying spaces.

For the case $m=1,2$ and $n=3,4$ there is the following natural commutative diagram with exact rows (see Brown-Loday [6]):

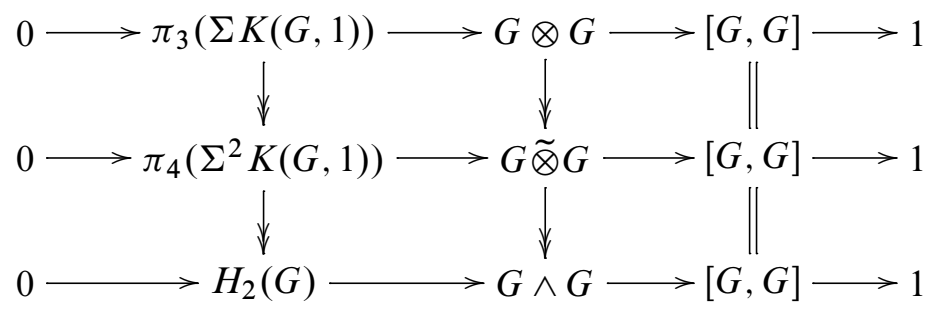


where $G \otimes G$ is the nonabelian square of $G$ in the sense of Brown-Loday [6], $G \widetilde{\otimes} G$ (resp. $G \wedge G$ ) is the quotient of $G \otimes G$ by the normal subgroup generated by elements $g \otimes h+h \otimes g$ (resp. $g \otimes g, g \in G$ ). In particular, for an abelian group $A$, there are natural isomorphisms

$$
\begin{aligned}
\pi_{3}(\Sigma K(A, 1)) & \simeq A \otimes A, \\
\pi_{4}\left(\Sigma^{2} K(A, 1)\right) & \simeq \pi_{2}^{S} K(A, 1) \simeq A \widetilde{\otimes} A .
\end{aligned}
$$

The purpose of this article is to determine the homotopy groups $\pi_{4}(\Sigma K(A, 1))$ and $\pi_{5}(\Sigma K(A, 1))$ for abelian groups $A$. In order to investigate the structure of these homotopy groups, we use the following methods of group theory and homotopy theory: derived functors, the Carlsson simplicial construction, the Baues-Goerss spectral sequence [4], homotopy decompositions and the methods of algebraic $K$-theory. The combination of these different methods provides an effective way for determining these homotopy groups. As reader will see, some our computations use commutator tricks in simplicial groups.

The homotopy group $\pi_{4}(\Sigma K(A, 1))$ as a functor on $A$ can be given as follows:

Theorem 1.1 (Theorem 3.4) Let $A$ be any abelian group. Then there is a natural short exact sequence

$$
\left(\Lambda^{2}(A) \otimes A\right)^{\oplus 2} \oplus A \otimes A \otimes \mathbb{Z} / 2 \hookrightarrow \pi_{4}(\Sigma K(A, 1)) \longrightarrow \operatorname{Tor}(A, A) .
$$

Moreover $\left(\Lambda^{2}(A) \otimes A\right)^{\oplus 2}$ is an (unnatural) summand of $\pi_{4}(\Sigma K(A, 1))$.

An interesting point of this theorem is that the functor $\pi_{4}(\Sigma K(A, 1))$ has $\operatorname{Tor}(A, A)$ as a natural quotient. For determining the structure of the group $\pi_{4}(\Sigma K(A, 1))$, one has to solve the group extension problem in Theorem 1.1. For finitely generated abelian groups $A$, we can solve this problem. Given a finitely generated abelian group $A$, let

$$
A=A_{1} \oplus \bigoplus_{\substack{r \geq 1 \\ p \text { is a prime }}} A_{p^{r}}
$$

be the primary decomposition of $A$, where $A_{1}$ is torsion free and $A_{p^{r}}$ is a free $\mathbb{Z} / p^{r}$-module.

Theorem 1.2 (Theorem 3.7) Let $A$ be any finitely generated abelian group. Let $A=A_{2} \oplus B$ with $B=A_{1} \oplus \bigoplus_{p^{r} \neq 2} A_{p^{r}}$. Then

$$
\begin{aligned}
\pi_{4}(\Sigma K(A, 1)) \cong \frac{1}{2}\left(A_{2} \otimes A_{2}\right) \oplus\left(A_{2} \otimes B\right)^{\oplus 2} \oplus B^{\otimes 2} \otimes \mathbb{Z} / 2 \oplus\left(A \otimes \Lambda^{2}(A)\right)^{\oplus 2} \\
\oplus \operatorname{Tor}\left(A_{2}, B\right)^{\oplus 2} \oplus \operatorname{Tor}(B, B),
\end{aligned}
$$

where $\frac{1}{2}\left(A_{2} \otimes A_{2}\right)$ is a free $\mathbb{Z} / 4$-module with rank of $\operatorname{dim}_{\mathbb{Z} / 2}\left(A_{2} \otimes A_{2}\right)$. 
One point of this theorem is that the (maximal) elementary 2-group summand $A_{2}$ of $A$ plays a key role in the group extension problem. Roughly speaking $A_{2} \otimes A_{2}$ is half down in the group $\pi_{4}(\Sigma K(A, 1))$.

As the applications of Theorems 1.1 and 1.2 , we are able to compute $\pi_{4}\left(M\left(\mathbb{Z} / 2^{r}, 2\right)\right)$ and their connections with $\pi_{4}\left(\Sigma K\left(\mathbb{Z} / 2^{r}, 1\right)\right)$. As the direct consequences, the homotopy groups $\pi_{4}\left(\Sigma \mathbb{R} \mathrm{P}^{n}\right)$ and $\pi_{4}\left(\Sigma K\left(\Sigma_{3}, 1\right)\right)$ are determined. (See Section 3.2 for the computations of these homotopy groups.)

For the homotopy group $\pi_{5}(\Sigma K(A, 1))$, as a functor, it can be described by two exact sequences given in diagram (4-1). Unfortunately it seems too complicated to produce a canonical functorial short exact sequence description for the functor $\pi_{5}(\Sigma K(A, 1))$ from diagram (4-1). For any finitely generated abelian group $A$, we determine $\pi_{5}(\Sigma K(A, 1))$ in an unfunctorial way by the following steps:

(1) From the Hopf fibration, $\pi_{5}(\Sigma K(A, 1)) \cong \pi_{5}(\Sigma K(A, 1) \wedge K(A, 1))$.

(2) Take a primary decomposition of $A$ and write $K(A, 1)$ as a product of copies of $S^{1}=K(\mathbb{Z}, 1)$ and $K\left(\mathbb{Z} / p^{r}, 1\right)$.

(3) By using the fact that

$$
\Sigma X \times Y \simeq \Sigma X \vee \Sigma Y \vee \Sigma X \wedge Y
$$

write $\Sigma K(A, 1) \wedge K(A, 1)$ as a wedge of the spaces in the form

$$
X=\Sigma^{m} K\left(\mathbb{Z} / p_{1}^{r_{1}}, 1\right) \wedge K\left(\mathbb{Z} / p_{2}^{r_{2}}, 1\right) \wedge \cdots \wedge K\left(\mathbb{Z} / p_{t}^{r_{t}}, 1\right)
$$

with $m+t \geq 3$ and $m \geq 1$.

(4) By applying the Hilton-Milnor Theorem, $\pi_{5}(\Sigma K(A, 1))$ becomes a summation of $\pi_{5}(X)$ for some $X$ in the above form.

For the spaces $X$ in the above form, it is contractible if $p_{i} \neq p_{j}$ for some $i \neq j$ and $\pi_{5}(X)$ can be determined in Proposition 4.2 for an odd prime $p$. The only difficult part is to compute $\pi_{5}(X)$ for $X$ given in the form

$$
X=\Sigma^{m} K\left(\mathbb{Z} / 2^{r_{1}}, 1\right) \wedge \cdots \wedge K\left(\mathbb{Z} / 2^{r_{t}}, 1\right)
$$

with $m+t \geq 3$ and $m \geq 1$. Our computations are then given case-by-case (Propositions 4.5-4.7 and Theorems 4.10-4.18), in which different methods are involved. An instructional example is as follows: 
Let $A=\mathbb{Z} \oplus \mathbb{Z} / 2$. According to (1), $\pi_{5}(\Sigma K(A, 1)) \cong \pi_{5}(\Sigma K(A, 1) \wedge K(A, 1))$. As in (3),

$$
\begin{aligned}
\Sigma K(A, 1) \wedge K(A, 1) & \simeq \Sigma\left(S^{1} \times \mathbb{R} \mathrm{P}^{\infty}\right) \wedge\left(S^{1} \times \mathbb{R} \mathrm{P}^{\infty}\right) \\
& \simeq \Sigma\left(S^{1} \vee \mathbb{R} \mathrm{P}^{\infty} \vee \Sigma \mathbb{R} \mathrm{P}^{\infty}\right) \wedge\left(S^{1} \vee \mathbb{R} \mathrm{P}^{\infty} \vee \Sigma \mathbb{R} \mathrm{P}^{\infty}\right) \\
= & S^{3} \vee \bigvee^{2} \Sigma^{2} \mathbb{R} \mathrm{P}^{\infty} \vee \bigvee^{2} \Sigma^{3} \mathbb{R} \mathrm{P}^{\infty} \vee \Sigma \mathbb{R} \mathrm{P}^{\infty} \wedge \mathbb{R} \mathrm{P}^{\infty} \\
& \vee \bigvee^{2} \Sigma^{2} \mathbb{R} \mathrm{P}^{\infty} \wedge \mathbb{R} \mathrm{P}^{\infty} \vee \Sigma^{3} \mathbb{R} \mathrm{P}^{\infty} \wedge \mathbb{R} \mathrm{P}^{\infty} .
\end{aligned}
$$

By applying the Hilton-Milnor Theorem as in (4), $\pi_{5}(\Sigma K(A, 1) \wedge K(A, 1))$ is a summation of

$$
\pi_{5}\left(S^{3}\right), \quad \pi_{5}\left(\Sigma^{2} \mathbb{R} \mathrm{P}^{\infty}\right), \quad \pi_{5}\left(\Sigma \mathbb{R} \mathrm{P}^{\infty} \wedge \mathbb{R} \mathrm{P}^{\infty}\right), \quad \pi_{5}\left(\Sigma\left(\mathbb{R} \mathrm{P}^{\infty}\right)^{\wedge 3}\right), \ldots
$$

with multiplicities. From Theorem 4.18, we have

$$
\pi_{5}\left(\Sigma^{2} \mathbb{R} \mathrm{P}^{\infty}\right)=\mathbb{Z} / 8
$$

and by Proposition 4.7 and Theorem 4.10, we have

$$
\pi_{5}\left(\Sigma \mathbb{R} \mathrm{P}^{\infty} \wedge \mathbb{R} \mathrm{P}^{\infty}\right)=\pi_{5}\left(\Sigma\left(\mathbb{R} \mathrm{P}^{\infty}\right)^{\wedge 3}\right)=\mathbb{Z} / 2^{\oplus 2} .
$$

The group $\pi_{5}(\Sigma K(\mathbb{Z} \oplus \mathbb{Z} / 2))$ will be determined by filling all possible summands with multiplicities.

As the applications of our computations on $\pi_{5}(\Sigma K(A, 1))$, we are able to determine $\pi_{5}\left(\Sigma \mathbb{R} \mathrm{P}^{n}\right)$ (Proposition 4.20) and $\pi_{5}\left(\Sigma K\left(\Sigma_{3}, 1\right)\right.$ ) (Proposition 4.21).

In Section 2 we recall certain facts from the homotopy theory, such as the Whitehead exact sequence, the Carlsson simplicial construction and describe a spectral sequence (2-9), which converges to $\pi_{*}\left(\Sigma^{m} K(A, 1)\right)$ for any abelian group $A$, with $E^{2}$-terms are given by the derived functors of certain polynomial functors. We illustrate how it works in Theorem 4.18 for computing

$$
\pi_{5}\left(\Sigma^{2} K\left(\mathbb{Z} / 2^{r}, 1\right)\right)= \begin{cases}\mathbb{Z} / 8, & \text { if } r=1, \\ \mathbb{Z} / 2^{r+1} \oplus \mathbb{Z} / 2, & \text { if } r>1 .\end{cases}
$$

The interesting point is of course how $\mathbb{Z} / 8$ shows up in the case $r=1$ while it becomes $\mathbb{Z} / 2^{r+1} \oplus \mathbb{Z} / 2$ for $r>1$. The proof is also based on the computations of the derived functors of the antisymmetric square $\widetilde{\otimes}^{2}$.

There is a natural relation between the problem considered and algebraic $K$-theory. Since the plus-construction $K(G, 1) \rightarrow K(G, 1)^{+}$is a homological equivalence, there 
is a natural weak homotopy equivalence

$$
\Sigma K(G, 1) \rightarrow \Sigma\left(K(G, 1)^{+}\right) .
$$

This defines the natural suspension map

$$
\pi_{n}\left(K(G, 1)^{+}\right) \rightarrow \pi_{n+1}\left(\Sigma\left(K(G, 1)^{+}\right)\right)=\pi_{n+1}(\Sigma K(G, 1))
$$

for $n \geq 1$. This map was studied by Baues and Conduché [3] in the case of a perfect group $G$. We consider the case $G=E(R)$, ie the group of elementary matrices over a ring $R$. In this case the natural map

$$
K_{3}(R)=\pi_{3}\left(K(E(R), 1)^{+}\right) \rightarrow \pi_{4}(\Sigma K(E(R), 1))
$$

is an isomorphism (Theorem 5.1). The natural relation to $K$-theory gives a way how to compute homotopy groups $\pi_{i}(\Sigma K(E(R), 1))$ for $i=4,5$ for some rings. For example, the case $G=\mathrm{SL}(\mathbb{Z})$ is considered. As an application of our methods, we also determine that $\pi_{4}\left(\Sigma K\left(A_{4}, 1\right)\right)=\mathbb{Z} / 4$ for the 4 -th alternating group $A_{4}$.

The article is organized as follows. We give a brief review for the quadratic functors and the simplicial resolutions in Section 2. The determination of $\pi_{4}(\Sigma K(A, 1))$ is given in Section 3, where the proofs of Theorems 1.1 and 1.2 are also given. In Section 4, we give case-by-case computations for $\pi_{5}(\Sigma K(A, 1))$. In Section 5, we give some relations to $K$-theory.

\section{The quadratic functors and the simplicial resolutions}

\subsection{Whitehead quadratic functor}

In [19, Chapter II], J H C Whitehead introduce the universal quadratic functor $\Gamma_{2}$ from abelian groups to abelian groups as follows: Let $A$ be any abelian group. Then $\Gamma_{2}(A)$ is the group generated by the symbols $\gamma(x)$, one for each $x \in A$, subject to the defining relations

(1) $\gamma(-x)=\gamma(x)$

(2) $\gamma(x+y+z)-\gamma(x+y)-\gamma(y+z)-\gamma(x+z)+\gamma(x)+\gamma(y)+\gamma(z)=0$.

Note According to [19, page 61], the group $\Gamma_{2}(A)$ is abelian and so the multiplication in $\Gamma_{2}(A)$ is denoted by + . Define

$$
\gamma(x, y)=\gamma(x+y)-\gamma(x)-\gamma(y) .
$$

The following proposition helps for determining the group $\Gamma_{2}(A)$. 
Proposition 2.1 [19, Theorem 5] Let $A$ be an abelian group with a basis $\left\{a_{i} \mid i \in I\right\}$ for a well-ordered index set $I$, and the defining relations $\left\{b_{\lambda} \equiv 0\right\}$. Then the group $\Gamma_{2}(A)$ is combinatorial defined by the set of symbolic generators $\gamma\left(a_{i}\right), i \in I$, and $\gamma\left(a_{i}, a_{j}\right), i, j \in I$ with $i<j$ with defining relations $\gamma\left(b_{\lambda}\right) \equiv 0$ and $\gamma\left(a_{i}, b_{\lambda}\right) \equiv 0$.

Example 2.2 We list some examples of the group $\Gamma_{2}(A)$. The first two examples are direct consequences of the above proposition.

(1) Let $A$ be a free abelian group with a basis $\left\{a_{i} \mid i \in I\right\}$ for a well-ordered index set $I$. Then $\Gamma_{2}(A)$ i s the free abelian group with a basis given by $\gamma\left(a_{i}\right), i \in I$, and $\gamma\left(a_{i}, a_{j}\right), i, j \in I$ with $i<j$.

(2) If $A$ is a cyclic group of finite order $m$ generated by $a_{1}$, then $\Gamma_{2}(A)$ is cyclic of order $m$ or $2 m$, according as $m$ is odd or even, generated by $\gamma\left(a_{1}\right)$.

(3) Let $A=\bigoplus_{i \in I} A_{i}$ for a well-ordered index set $I$. Then [19, Theorem 7]

$$
\Gamma_{2}(A) \cong \bigoplus_{i \in I} \Gamma_{2}\left(A_{i}\right) \oplus \bigoplus_{\substack{i, j \in I \\ i<j}} A_{i} \otimes A_{j}
$$

(4) For a general abelian group $A$, there is a short exact sequence [9, formula (13.8), page 93]

$$
A \otimes A \stackrel{t}{\longrightarrow} \Gamma_{2}(A) \longrightarrow A \otimes \mathbb{Z} / 2,
$$

where $t(a \otimes b)=\gamma(a, b)=\gamma(a+b)-\gamma(a)-\gamma(b)$.

\subsection{Lower homology of $K(A, 2)$}

The homology of Eilenberg-Mac Lane spaces $K(A, n)$ has been studied in the classical reference of Eilenberg and Mac Lane [9] and other papers. See also [5] for the functorial description of homology groups of $K(A, 2)$ in all dimensions.

Lemma 2.3 [9, Theorems 20.5 and 21.1] Let $A$ be any abelian group. Then

(1) $H_{2}(K(A, 2))=A$;

(2) $H_{3}(K(A, 2))=0$;

(3) $H_{4}(K(A, 2))=\Gamma_{2}(A)$.

The homology $H_{5}(K(A, 2))$ becomes a special functor on $A$. Let $R_{2}(A)$ denote $H_{5}(K(A, 2))$. The group $R_{2}(A)$ for finitely generated abelian group $A$ can be computed as follows [9, Section 22]: 
(1) If $A$ is a cyclic group of order infinite or odd, then $R_{2}(A)=0$.

(2) If $A=\mathbb{Z} / 2^{r} \mathbb{Z}$ with $r \geq 1$, then $R_{2}(A) \cong \mathbb{Z} / 2$.

(3) Let $A=A_{1} \oplus A_{2}$. Then $K(A, 2) \simeq K\left(A_{1}, 2\right) \times K\left(A_{2}, 2\right)$. By using Künneth theorem together with the fact that $H_{1}(K(A, 2))=H_{3}(K(A, 2))=0$ from Lemma 2.3, we have

$H_{5}(K(A, 2)) \cong H_{5}\left(K\left(A_{1}, 2\right)\right) \oplus H_{5}\left(K\left(A_{2}, 2\right)\right) \oplus \operatorname{Tor}\left(H_{2}\left(K\left(A_{1}, 2\right)\right), H_{2}\left(K\left(A_{2}, 2\right)\right)\right)$.

Thus

$$
R_{2}\left(A_{1} \oplus A_{2}\right) \cong R_{2}\left(A_{1}\right) \oplus R_{2}\left(A_{2}\right) \oplus \operatorname{Tor}\left(A_{1}, A_{2}\right) .
$$

Recall the definition of the derived functors in the sense of Dold-Puppe [8]. Let $F$ be an endofunctor in the category of abelian groups and $A$ an abelian group. Take a projective resolution $P_{*} \rightarrow A$. Let $N^{-1}$ be the inverse map to the normalization map due to Dold-Kan. Then $N^{-1} P_{*}$ is a free simplicial resolution of $A$. Then, the $i-$ th derived functor of $F$ applied to the abelian group $A$, is defined as follows:

$$
L_{i} F(A)=\pi_{i}\left(F\left(N^{-1} P_{*}\right)\right), \quad i \geq 0 .
$$

It is a well-known fact that this definition does not depend on a choice of a projective resolution. In this notation, one has a natural isomorphism

$$
R_{2}(A)=L_{1} \Gamma_{2}(A) .
$$

\subsection{Whitehead exact sequence}

Let $X$ be a $(r-1)$-connected CW-complex, $r \geq 2$. There is the following long exact sequence of abelian groups [19, Theorem 1]:

$$
\cdots \rightarrow H_{n+1}(X) \rightarrow \Gamma_{n}(X) \rightarrow \pi_{n}(X) \stackrel{h_{n}}{\rightarrow} H_{n}(X) \rightarrow \Gamma_{n-1}(X) \rightarrow \cdots,
$$

where $\Gamma_{n}(X)=\operatorname{Im}\left(\pi_{n}\left(\mathrm{sk}_{n-1}(X)\right) \rightarrow \pi_{n}\left(\mathrm{sk}_{n}(X)\right)\right)$ (here $\mathrm{sk}_{i}(X)$ is the $i$-th skeleton of $X), h_{n}$ is the $n$-th Hurewicz homomorphism.

The Hurewicz theorem is equivalent to the statement $\Gamma_{i}(X)=0, i \leq r$. J HC Whitehead computed the term $\Gamma_{r+1}(X)$ : In the following theorem, assertion (1) was given in [19, Theorem 14] and assertion (2) was given the earlier paper [18]. According to the remarks in the end of [19, Section 14], assertion (2) has been discussed by G W Whitehead [17] as well.

Theorem 2.4 Let $X$ be a $(r-1)$-connected $C W$-complex with $r \geq 2$. Then

(1) If $r=2$, then $\Gamma_{3}(X) \cong \Gamma_{2}\left(\pi_{2}(X)\right)$;

(2) If $r>2$, then $\Gamma_{r+1}(X) \cong \pi_{r}(X) \otimes \mathbb{Z} / 2$. 
The isomorphism $\Gamma_{2}\left(\pi_{2}(X)\right) \rightarrow \Gamma_{3}(X)$ is constructed as follows: Let $\eta: S^{3} \rightarrow S^{2}$ be the Hopf map and let $x \in \pi_{2}(X)$ be written as the composite

$$
S^{2} \stackrel{\tilde{x}}{\longrightarrow} \operatorname{sk}_{2}(X) \subset \mathrm{sk}_{3}(X) \text {. }
$$

Then the composite

$$
S^{3} \stackrel{\eta}{\longrightarrow} S^{2} \stackrel{\tilde{x}}{\longrightarrow} \operatorname{sk}_{2}(X) \subset \operatorname{sk}_{3}(X)
$$

defines an element $\eta^{*}(x) \in \Gamma_{3}(X)$. According to [19, Section 13], the mapping

$$
\eta_{1}: \Gamma_{2}\left(\pi_{2}(X)\right) \rightarrow \Gamma_{3}(X), \quad \gamma(x) \mapsto \eta^{*}(x),
$$

is a well-defined isomorphism of groups. The construction of the isomorphism $\pi_{r}(X) \otimes$ $\mathbb{Z} / 2 \rightarrow \Gamma_{r+1}(X)$ in assertion (2) is similar.

Recall the description of the functors $\Gamma_{r+2}(X)$ due to H-J Baues [2]. Consider the third super-Lie functor

$$
\mathcal{L}_{s}^{3}: \mathrm{Ab} \rightarrow \mathrm{Ab}
$$

defined as

$$
\mathcal{L}_{S}^{3}(A)=\operatorname{im}\{A \otimes A \otimes A \stackrel{l}{\rightarrow} A \otimes A \otimes A\}
$$

where

$l(a \otimes b \otimes c)=\{a, b, c\}:=a \otimes b \otimes c+b \otimes a \otimes c-c \otimes a \otimes b-c \otimes b \otimes a, a, b, c \in A$. Observe that $\mathcal{L}_{S}^{3}(A)=\operatorname{ker}\left\{A \otimes \Lambda^{2}(A) \stackrel{r}{\rightarrow} \Lambda^{3}(A)\right\}$, where $\Lambda^{i}(A)$ is the $i$-th exterior power of $A$ and the map $r$ is given as

$$
r(a \otimes b \wedge c)=a \wedge b \wedge c, \quad a, b, c \in A .
$$

Let the complex $X$ be simply connected. Given an abelian group $A$, define the map

$$
q: \Gamma_{2}(A) \otimes A \rightarrow \mathcal{L}_{s}^{3}(A) \oplus \Gamma_{2}(A) \otimes \mathbb{Z} / 2
$$

by setting

$$
q\left(\gamma_{2}(a) \otimes b\right)=-\{b, a, a\}+\left(\gamma_{2}(a+b)-\gamma_{2}(a)-\gamma_{2}(b)\right) \otimes 1, \quad a, b \in A .
$$

Define the group $\Gamma_{2}^{2} X=\Gamma_{2}^{2}\left(\Gamma_{2}\left(\pi_{2} X\right) \rightarrow \pi_{3} X\right)$ as the pushout:

$$
\begin{gathered}
\Gamma_{2}\left(\pi_{2}(X)\right) \otimes\left(\pi_{2}(X) \oplus \mathbb{Z} / 2\right) \stackrel{q \oplus \mathrm{id}}{\longrightarrow} \mathcal{L}_{S}^{3}\left(\pi_{2}(X)\right) \oplus \Gamma_{2}\left(\pi_{2}(X)\right) \otimes \mathbb{Z} / 2 \\
\downarrow_{\eta_{1} \otimes \text { id }} \\
\pi_{3}(X) \otimes\left(\pi_{2}(X) \oplus \mathbb{Z} / 2\right) \longrightarrow \Gamma_{2}^{2}(X)
\end{gathered}
$$

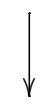


Theorem 2.5 [2, Theorem 3.1] Let $X$ be a $(r-1)$-connected $C W$-complexes with $r \geq 2$.

(1) If $r=2$, then there is a natural short exact sequence

$$
0 \rightarrow \Gamma_{2}^{2}(X) \rightarrow \Gamma_{4}(X) \rightarrow R_{2}\left(\pi_{2}(X)\right) \rightarrow 0 .
$$

(2) If $r=3$, then there is a natural exact sequence

$$
0 \rightarrow \pi_{4}(X) \otimes \mathbb{Z} / 2 \oplus \Lambda^{2}\left(\pi_{3}(X)\right) \rightarrow \Gamma_{5}(X) \rightarrow \operatorname{Tor}\left(\pi_{3}(X), \mathbb{Z} / 2\right) \rightarrow 0 .
$$

(3) If $r \geq 4$, there is a natural exact sequence

$$
0 \rightarrow \pi_{r+1}(X) \otimes \mathbb{Z} / 2 \rightarrow \Gamma_{r+2}(X) \rightarrow \operatorname{Tor}\left(\pi_{r}(X), \mathbb{Z} / 2\right) \rightarrow 0 .
$$

Let $A$ be an abelian group. Consider the Hurewicz homomorphism

$$
h_{*}: \pi_{*}(\Sigma K(A, 1)) \rightarrow \widetilde{H}_{*}(\Sigma K(A, 1))=\widetilde{H}_{*-1}(K(A, 1))=\widetilde{H}_{*-1}(A) .
$$

Since $H_{*}(K(A, 1))$ is graded commutative ring, the inclusion

$$
A=H_{1}(K(A, 1)) \smile H_{*}(K(A, 1))
$$

induces a ring homomorphism

$$
\lambda: \Lambda(A) \longrightarrow H_{*}(K(A, 1)) .
$$

By [9, Theorem 19.3], $\lambda$ is a monomorphism and so we may consider $\Lambda^{n}(A) \subseteq$ $H_{n}(K(A, 1))=H_{n}(A)$.

Lemma 2.6 For every abelian group $A$, the Hurewicz image

$$
\operatorname{Im}\left(h_{n+1}: \pi_{n+1}(\Sigma K(A, 1)) \rightarrow H_{n}(A)\right)
$$

contains the subgroup $\Lambda^{n}(A)$.

Proof From the naturality, it suffices to show that the statement holds for a free abelian group $A$.

When $A$ is a free abelian group, then $K(A, 1)$ is a (weak) Cartesian product of the circles. Thus $\Sigma K(A, 1)$ is a wedge of spheres from the suspension splitting that

$$
\Sigma X \times Y \simeq \Sigma X \vee \Sigma Y \vee \Sigma X \wedge Y
$$

and so the Hurewicz homomorphism induces an epimorphism

$$
h_{*}: \pi_{n+1}(\Sigma K(A, 1)) \longrightarrow H_{n}(A)=\Lambda^{n}(A)
$$

for a free abelian group $A$. This finishes the proof. 


\subsection{Carlsson construction}

Let $G_{*}$ be a simplicial group and $X$ a pointed simplicial set with a base point $*$. Consider the simplicial group $F^{G_{*}}(X)$ defined as

$$
F^{H}(X)_{n}=\coprod_{x \in X_{n}}\left(G_{n}\right)_{x}
$$

ie in each degree $F^{G_{*}}(X)_{n}$ is the free product of groups $G_{n}$ numerated by elements of $X_{n}$ modulo $\left(G_{n}\right)_{*}$, with the canonical choice of face and degeneracy morphisms. It is proved in [7] that the geometric realization $\left|F^{G_{*}}(X)\right|$ is homotopy equivalent to the loop space $\Omega(|X| \wedge B|G|)$. The main example we will consider is the simplicial circle $X=S^{1}$ with

$$
S_{0}^{1}=\{*\}, S_{1}^{1}=\{*, \sigma\}, S_{2}^{1}=\left\{*, s_{0} \sigma, s_{1} \sigma\right\}, \ldots, S_{n+1}^{1}=\left\{*, x_{0}, \ldots, x_{n}\right\},
$$

where $x_{i}=s_{n} \cdots \hat{s}_{i} \cdots s_{0} \sigma$ and the simplicial group $G_{*}$ with $G_{n}=G$ for a given group $G$, with identity homomorphisms as all face and degeneracy maps. In this case we use the notation $F^{G}(X)=F^{G}(X)$. One has a homotopy equivalence

$$
\left|F^{G}\left(S^{1}\right)\right| \simeq \Omega \Sigma K(G, 1) .
$$

The group $F^{G}\left(S^{1}\right)_{n}$ is the $n$-fold free product of $G$ :

$$
F^{G}\left(S^{1}\right)_{1}=G, F^{G}\left(S^{1}\right)_{2}=G * G, F^{G}\left(S^{1}\right)_{3}=G * G * G, \ldots
$$

We can formally identify $G * G$ with $s_{0} G * s_{1} G, G * G * G$ with $s_{1} s_{0} G * s_{2} s_{0} G * s_{2} s_{1} G$, etc, and to define naturally the face and degeneracy maps:

$$
F^{G_{*}}\left(S^{1}\right): \quad \ldots \underset{\rightleftarrows}{\stackrel{\rightleftarrows}{\rightleftarrows}} G * G * G \underset{\rightleftarrows}{\rightleftarrows} G * G \underset{\rightleftarrows}{\rightleftarrows} G .
$$

Remark Consider the second term $F^{G}\left(S^{1}\right)_{2}=G * G$ and face morphisms $d_{0}, d_{1}$, $d_{2}: G * G=s_{0}(G) * s_{1}(G) \rightarrow G$ defined as

$$
d_{0}:\left\{\begin{array}{l}
s_{0}(g) \mapsto g, \\
s_{1}(g) \mapsto 1,
\end{array} \quad d_{1}:\left\{\begin{array}{l}
s_{0}(g) \mapsto g, \\
s_{1}(g) \mapsto g,
\end{array} \quad d_{2}:\left\{\begin{array}{l}
s_{0}(g) \mapsto 1, \\
s_{1}(g) \mapsto g .
\end{array}\right.\right.\right.
$$

There is a natural commutative diagram

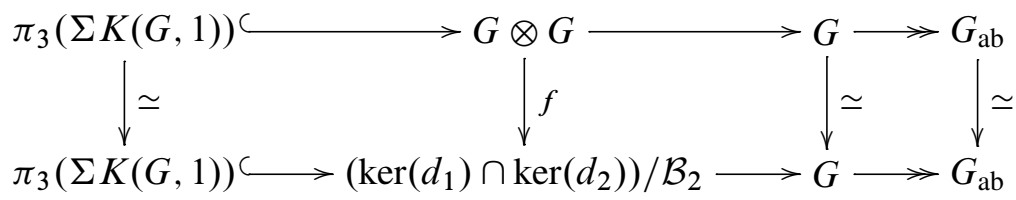


where $\mathcal{B}_{2}$ is the 2-boundary subgroup of $G * G$ and the map $f$ is defined as ${ }^{1}$

$$
f(g \otimes h)=\left[s_{0}(g) s_{1}(g)^{-1}, s_{0}(h)\right] \cdot \mathcal{B}_{2}
$$

There is a natural description of the 2-boundary (see Ellis and Mikhailov [10], for example):

$\mathcal{B}_{2}=\left[\operatorname{ker}\left(d_{0}\right), \operatorname{ker}\left(d_{1}\right) \cap \operatorname{ker}\left(d_{2}\right)\right]\left[\operatorname{ker}\left(d_{1}\right), \operatorname{ker}\left(d_{2}\right) \cap \operatorname{ker}\left(d_{0}\right)\right]$

$\cdot\left[\operatorname{ker}\left(d_{2}\right), \operatorname{ker}\left(d_{0}\right) \cap \operatorname{ker}\left(d_{1}\right)\right]$.

Diagram (2-5) implies that $f$ is a natural isomorphism.

In the case $G=\mathbb{Z}$, the simplicial group $F^{G}\left(S^{1}\right)$ is identical to the Milnor construction $F\left(S^{1}\right)$, with $F\left(S^{1}\right)_{n}$ a free group of rank $n$, for $n \geq 1$ :

$$
F\left(S^{1}\right): \quad \ldots \underset{\rightleftarrows}{\stackrel{\rightleftarrows}{\rightleftarrows}} F_{3} \underset{\rightleftarrows}{\rightleftarrows} F_{2} \underset{\rightleftarrows}{\rightleftarrows}
$$

In this case there is a homotopy equivalence

$$
\left|F\left(S^{1}\right)\right| \simeq \Omega S^{2}
$$

and the construction $F\left(S^{1}\right)$ provides a combinatorial model for the computation of homotopy groups of the 2 -sphere $S^{2}$. The construction $F\left(S^{1}\right)$ was studied from the group-theoretical point of view in $\mathrm{Wu}$ [20]. It is easy to find the simplicial generators of the homotopy classes of $\pi_{i}\left(F\left(S^{1}\right)\right)=\pi_{i+1}\left(S^{2}\right)$ for $i=3,4,5$. In order to find these simplicial generators, consider the sequence of maps between Milnor simplicial constructions $F\left(S^{4}\right) \rightarrow F\left(S^{3}\right) \rightarrow F\left(S^{2}\right) \rightarrow F\left(S^{1}\right)$ such that the induced homomorphisms $\mathbb{Z}=\pi_{2}\left(F\left(S^{2}\right)\right) \rightarrow \pi_{2}\left(F\left(S^{1}\right)\right)=\mathbb{Z}$ and $\mathbb{Z}=\pi_{3}\left(F\left(S^{3}\right)\right) \rightarrow \pi_{3}\left(F\left(S^{2}\right)\right)=\mathbb{Z} / 2$ are

\footnotetext{
${ }^{1}$ We use the standard commutator relations: $[g, h]=g^{-1} h^{-1} g h$
} 
epimorphisms and define the homotopy classes of $\pi_{3}\left(S^{2}\right)$ and $\pi_{4}\left(S^{3}\right)$ respectively.

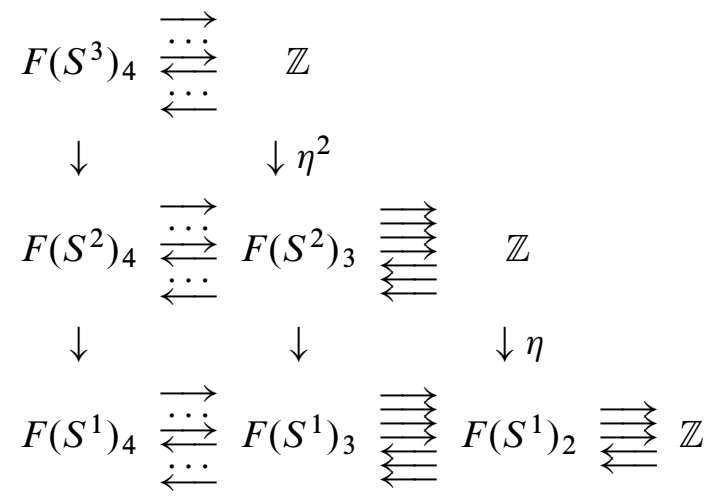

For $n \geq 3$, the homotopy class of $\pi_{n}\left(S^{n-1}\right)$ defined as $\pi_{n-1}\left(F\left(S^{n-2}\right)\right)$ is generated by

$$
\left[s_{0}\left(\sigma_{n-2}\right), s_{1}\left(\sigma_{n-2}\right)\right]
$$

in $F\left(S^{n-2}\right)_{n-1}$ (see Wu [20]), where $\sigma_{n-2}$ is a generator of $F\left(S^{n-2}\right)_{n-2}=\mathbb{Z}$. That is, we can define the simplicial suspension maps

$$
\begin{aligned}
\eta^{i}: F\left(S^{i+1}\right)_{i+1} & \rightarrow F\left(S^{i}\right)_{i+1} \\
\eta^{i}: \sigma_{i+1} & \rightarrow\left[s_{0}\left(\sigma_{i}\right), s_{1}\left(\sigma_{i}\right)\right], \quad i \geq 1 .
\end{aligned}
$$

by

Since the generators of $\pi_{i}\left(S^{2}\right)$ are presented by suspensions over Hopf fibration for $i=3,4,5$, the simplicial generators of $\pi_{i}\left(F\left(S^{1}\right)\right), i=2,3,4$ are given by the following elements:

$$
\begin{aligned}
& w_{2}\left(x_{0}, x_{1}\right)=\left[x_{0}, x_{1}\right] \\
& w_{3}\left(x_{0}, x_{1}, x_{2}\right)=\left[\left[x_{0}, x_{2}\right],\left[x_{0}, x_{1}\right]\right] \\
& w_{4}\left(x_{0}, x_{1}, x_{2}, x_{3}\right)=\left[\left[\left[x_{0}, x_{3}\right],\left[x_{0}, x_{1}\right]\right],\left[\left[x_{0}, x_{2}\right],\left[x_{0}, x_{1}\right]\right]\right] .
\end{aligned}
$$

Here we use the natural notation

$$
x_{j}:=s_{i} \cdots \hat{s}_{j} \cdots s_{0}\left(\sigma_{1}\right), \quad j=0, \ldots, i
$$

for the basis elements in $F\left(S^{1}\right)_{i+1}^{2}$.

\footnotetext{
${ }^{2}$ One can continue the process of construction of elements $w_{n+1}\left(x_{0}, \ldots, x_{n}\right)$ by the following law: $w_{n+1}\left(x_{0}, \ldots, x_{n}\right)=\left[w_{n}\left(x_{0}, \ldots, \hat{x}_{n-1}, x_{n}\right), w_{n}\left(x_{0}, \ldots, x_{n-1}\right)\right]$. In this case, the 16-commutator bracket $w_{5}\left(x_{0}, \ldots, x_{4}\right)$ corresponds to the element of order 2 in $\pi_{6}\left(S^{2}\right)$, but the 32-commutator bracket $w_{6}\left(x_{0}, \ldots, x_{5}\right)$ lies in the simplicial boundary subgroup $\mathcal{B} F\left(S^{1}\right)_{6}$ [9]. The construction of a simplicial generator of the 3 -torsion in $\pi_{6}\left(S^{2}\right)$ is more tricky: it is possible to find its simplicial representative which is a product of six brackets of the commutator weight six.
} 


\subsection{Spectral sequence}

Consider an abelian group $A$ and its two-step flat resolution

$$
0 \rightarrow A_{1} \rightarrow A_{0} \rightarrow A \rightarrow 0
$$

By Dold-Kan correspondence, we obtain the following free abelian simplicial resolution of $A$ :

$$
N^{-1}\left(A_{1} \hookrightarrow A_{0}\right): \quad \ldots \rightleftarrows A_{1} \oplus s_{0}\left(A_{0}\right) \rightleftarrows A_{0} .
$$

Applying Carlsson construction to the resolution $N^{-1}\left(A_{1} \hookrightarrow A_{0}\right)$, we obtain the following bisimplicial group:

$$
\begin{aligned}
& F^{N^{-1}\left(A_{1} \hookrightarrow A_{0}\right)_{2}}\left(S^{n}\right)_{3} \underset{\rightleftarrows}{\rightleftarrows} F^{N^{-1}\left(A_{1} \hookrightarrow A_{0}\right)_{2}}\left(S^{n}\right)_{2} \rightleftarrows N^{-1}\left(A_{1} \hookrightarrow A_{0}\right)_{2} \\
& \downarrow \downarrow \downarrow \uparrow \uparrow \\
& \downarrow \downarrow \downarrow \uparrow \uparrow \\
& \downarrow \downarrow \downarrow \uparrow \uparrow
\end{aligned}
$$

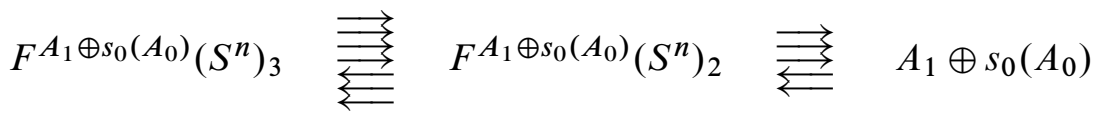

$$
\begin{aligned}
& \downarrow \downarrow \uparrow \\
& \downarrow \downarrow \uparrow \\
& \downarrow \downarrow \uparrow \\
& F^{A_{0}}\left(S^{n}\right)_{3} \\
& \underset{\equiv}{\rightleftarrows} \\
& F^{A_{0}}\left(S^{n}\right)_{2} \\
& \rightleftarrows
\end{aligned}
$$

Here the $m$-th horizontal simplicial group is Carlsson construction $F^{N^{-1}\left(A_{1} \hookrightarrow A_{0}\right)_{m}}\left(S^{n}\right)$. By the result of Quillen [14], we obtain the following spectral sequence:

$$
E_{p, q}^{2}=\pi_{q}\left(\pi_{p}\left(\Sigma^{n} K\left(N^{-1}\left(A_{1} \hookrightarrow A_{0}\right), 1\right)\right) \Longrightarrow \pi_{p+q}\left(\Sigma^{n} K(A, 1)\right) .\right.
$$

Consider now a nonabelian analog of this spectral sequence, for $n=1$. Suppose now that a group $G$ is arbitrary, not necessary abelian. Consider a simplicial resolution of $G$ :

$$
G_{\bullet} \rightarrow G
$$


ie $G_{\bullet}$ is a simplicial group with $\pi_{0}\left(G_{\bullet}\right)=G, \pi_{i}\left(G_{\bullet}\right)=0, i>0$. Consider the following bisimplicial group:

$$
\begin{aligned}
& G_{2} * G_{2} * G_{2} \underset{\rightrightarrows}{\rightleftarrows} G_{2} * G_{2} \underset{\Xi}{\rightleftarrows} G_{2} \\
& \downarrow \downarrow \downarrow \uparrow \uparrow \quad \downarrow \downarrow \downarrow \uparrow \uparrow \quad \downarrow \downarrow \downarrow \uparrow \uparrow \\
& G_{1} * G_{1} * G_{1} \underset{\Xi}{\rightleftarrows} G_{1} * G_{1} \rightleftarrows G_{1} \\
& \downarrow \downarrow \uparrow \quad \downarrow \downarrow \uparrow \quad \downarrow \downarrow \uparrow \\
& G_{0} * G_{0} * G_{0} \underset{\Xi}{\rightleftarrows} G_{0} * G_{0} \rightleftarrows G_{0}
\end{aligned}
$$

Again, by the result of Quillen [14], we obtain the following spectral sequence:

$$
E_{p, q}^{2}=\pi_{q}\left(\pi_{p}\left(\Sigma K\left(G_{\bullet}, 1\right)\right)\right) \Longrightarrow \pi_{p+q}(\Sigma K(G, 1))
$$

If $G_{\bullet}$ is a free simplicial resolution, the spectral sequence (2-10) contains a lot of canonical differentials of a complicated nature:

$$
\pi_{2}\left(\mathcal{L}_{S}^{3}\left(\left(G_{\bullet}\right)_{\mathrm{ab}}\right) \oplus \Gamma_{2}\left(\left(G_{\bullet}\right)_{\mathrm{ab}}\right) \otimes \mathbb{Z} / 2\right)
$$

$$
\pi_{1}\left(\mathcal{L}_{S}^{3}\left(\left(G_{\bullet}\right)_{\mathrm{ab}}\right) \oplus \Gamma_{2}\left(\left(G_{\bullet}\right)_{\mathrm{ab}}\right) \otimes \mathbb{Z} / 2\right)
$$

$$
\mathcal{L}_{S}^{3}\left(G_{\mathrm{ab}}\right) \oplus \Gamma_{2}\left(G_{\mathrm{ab}}\right) \otimes \mathbb{Z} / 2
$$

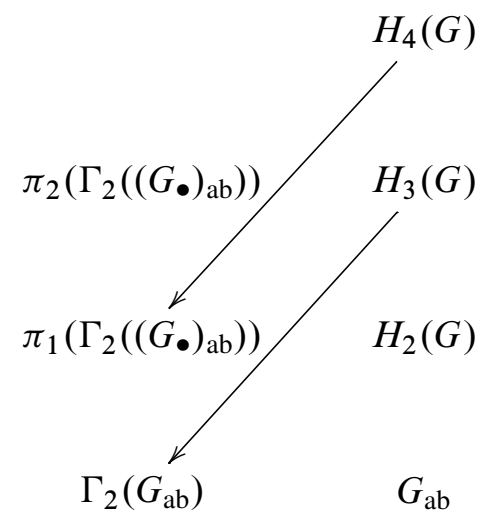




\section{On group $\pi_{4}(\Sigma K(A, 1))$}

\subsection{The group $\pi_{4}(\Sigma K(A, 1))$ for an abelian group $A$}

Let $A$ be an abelian group. Consider the homotopy commutative diagram of fibre sequences

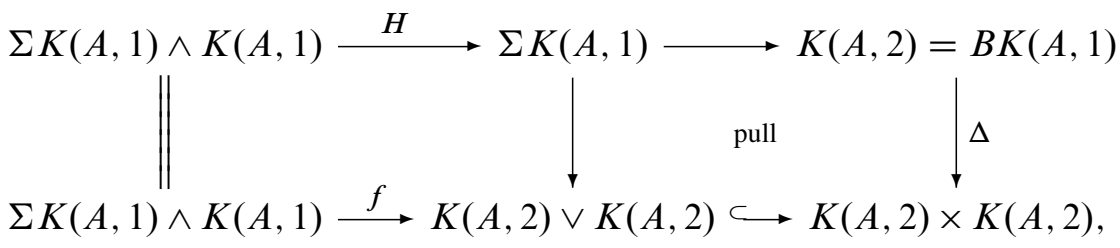

where $H$ is the Hopf fibration. Thus we have the following lemma:

Lemma 3.1 There are isomorphisms

$$
\pi_{n}(\Sigma K(A, 1) \wedge K(A, 1)) \cong \pi_{n}(\Sigma K(A, 1)) \cong \pi_{n}(K(A, 2) \vee K(A, 2))
$$

for $n \geq 3$. In particular, $\pi_{3}(\Sigma K(A, 1)) \cong \pi_{3}(\Sigma K(A, 1) \wedge K(A, 1)) \cong A \otimes A$.

By Lemma 2.3, we have:

Lemma 3.2 The lower homology of the wedge $K(A, 2) \vee K(A, 2)$ are the following:

$$
\begin{aligned}
& H_{2}(K(A, 2) \vee K(A, 2))=A \oplus A, \\
& H_{3}(K(A, 2) \vee K(A, 2))=0, \\
& H_{4}(K(A, 2) \vee K(A, 2))=\Gamma_{2}(A) \oplus \Gamma_{2}(A), \\
& H_{5}(K(A, 2) \vee K(A, 2))=R_{2}(A) \oplus R_{2}(A) .
\end{aligned}
$$

Lemma 3.3 The Hurewicz image

$$
h_{n}: \pi_{n}(K(A, 2) \vee K(A, 2)) \longrightarrow H_{n}(K(A, 2) \vee K(A, 2))
$$

is zero for $n \geq 3$.

Proof The commutative diagram

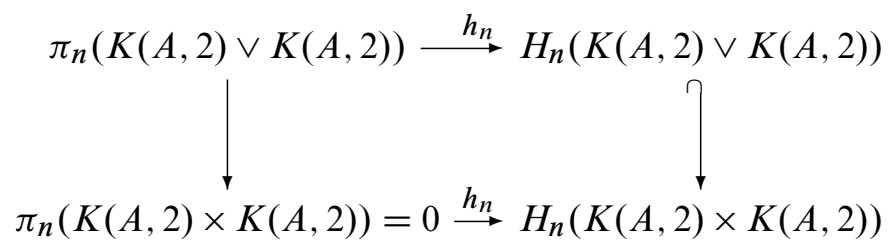

implies the assertion. 
Theorem 3.4 Let $A$ be any abelian group. Then there is a natural short exact sequence $\left(\Lambda^{2}(A) \otimes A\right)^{\oplus 2} \oplus A \otimes A \otimes \mathbb{Z} / 2 \hookrightarrow \pi_{4}(\Sigma K(A, 1)) \longrightarrow \operatorname{Tor}(A, A)$.

Moreover $\left(\Lambda^{2}(A) \otimes A\right)^{\oplus 2}$ is an (unnatural) summand of $\pi_{4}(\Sigma K(A, 1))$.

Proof Let $X=K(A, 2) \vee K(A, 2)$ and let $Y=K(A, 2) \times K(A, 2)=K(A \oplus A, 2)$. From Lemmas 3.2 and 3.3, there is a short exact sequence

$$
R_{2}(A) \oplus R_{2}(A) \subset \Gamma_{4}(X) \longrightarrow \pi_{4}(X) .
$$

The inclusion $j: X=K(A, 2) \vee K(A, 2) \smile Y=K(A, 2) \times K(A, 2)$ induces a commutative diagram

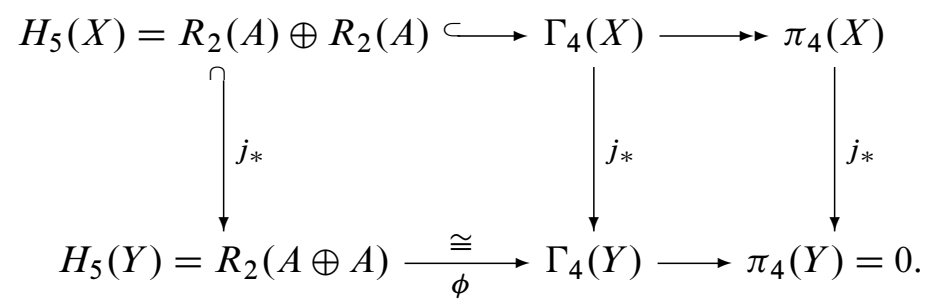

By formula (2-1),

$$
H_{5}(K(A, 2) \times K(A, 2))=H_{5}(K(A \oplus A, 2))=R_{2}(A) \oplus R_{2}(A) \oplus \operatorname{Tor}(A, A)
$$

and so the cokernel of $j_{*}: H_{5}(X) \rightarrow H_{5}(Y)$ is $\operatorname{Tor}(A, A)$. On the other hand, from Theorem 2.5(1), there is a commutative diagram of short exact sequences

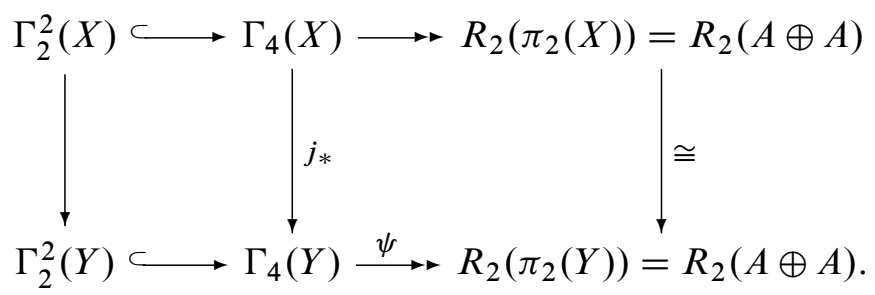

The composite

$$
\psi \circ \phi: R_{2}(A \oplus A) \stackrel{\cong}{\longrightarrow}{ }_{\phi} \Gamma_{4}(Y) \longrightarrow \psi R_{2}(A \oplus A)
$$

is a natural self epimorphism for any abelian group $A$. It is an isomorphism for any finitely generated abelian $A$ and so an isomorphism for any abelian group $A$ by considering the direct limit. Thus $j_{*}: \Gamma_{4}(X) \rightarrow \Gamma_{4}(Y)$ is an epimorphism and, from diagram (3-2), there is a short exact sequence

$$
\Gamma_{2}^{2}(X) \subset \pi_{4}(X) \longrightarrow \operatorname{Tor}(A, A) .
$$


Let $Z=\Sigma K(A, 1) \wedge K(A, 1)$ and let $f: Z \rightarrow X$ be the map in diagram (3-1). Consider the commutative diagram

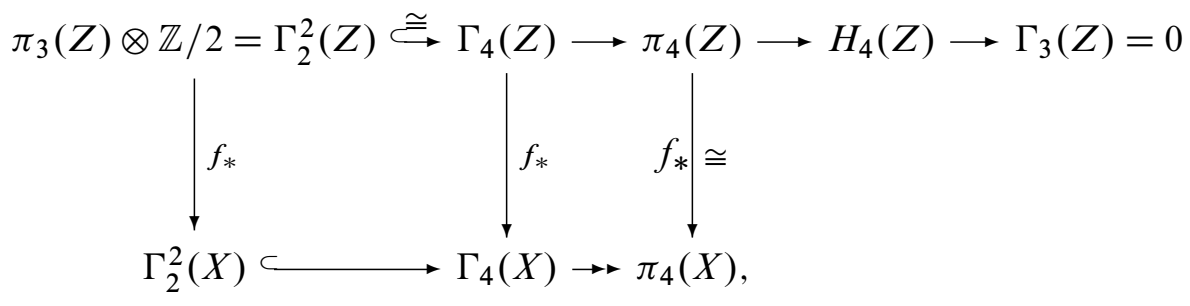

where $\Gamma_{2}^{2}(Z) \rightarrow \Gamma_{4}(Z)$ is an isomorphism because its cokernel $R_{2}\left(\pi_{2}(Z)\right)=0$. From the definition (2-4) of the functor $\Gamma_{2}^{2}$,

$$
f_{*}: \Gamma_{2}^{2}(Z) \longrightarrow \Gamma_{2}^{2}(X)
$$

is a monomorphism with retracting homomorphism $\phi^{\prime}: \Gamma_{2}^{2}(X) \rightarrow \Gamma_{2}^{2}(Z)$. By the short exact sequence (3-3), $\Gamma_{4}(Z) \rightarrow \pi_{4}(Z)$ is a monomorphism and so there is a short exact sequence

(3-5) $A \otimes A \otimes \mathbb{Z} / 2=\Gamma_{4}(Z) \subset \pi_{4}(Z) \longrightarrow H_{4}(Z)=H_{3}(K(A, 1) \wedge K(A, 1))$.

Note that $H_{1}(K(A, 1))=A$ and $H_{2}(K(A, 1))=\Lambda^{2}(A)$. By the Künneth theorem, there is a natural short exact sequence

$$
\left(A \otimes \Lambda^{2}(A)\right)^{\oplus 2} \subset H_{4}(Z) \stackrel{\psi^{\prime}}{\longrightarrow} \operatorname{Tor}(A, A)
$$

Consider the composite

$$
\begin{aligned}
\theta_{A}: \Gamma_{2}^{2}(X)=\Gamma_{2}^{2}\left(\Gamma_{2}(A \oplus A)\right. & \rightarrow A \otimes A) \\
& \hookrightarrow \pi_{4}(X) \stackrel{f_{*}^{-1}}{\longrightarrow} \pi_{4}(Z) \longrightarrow H_{4}(Z) \stackrel{\psi^{\prime}}{\longrightarrow} \operatorname{Tor}(A, A),
\end{aligned}
$$

which is natural on any abelian group $A$. If $A$ is a free abelian group, then $\theta_{A}=0$. For any abelian group $A$, choose any free abelian group $A_{0}$ with an epimorphism $g: A_{0} \rightarrow A$. From the definition (2-4) of $\Gamma_{2}^{2}$,

$$
\Gamma_{2}^{2}(g): \Gamma_{2}^{2}\left(\Gamma_{2}\left(A_{0} \oplus A_{0}\right) \rightarrow A_{0} \otimes A_{0}\right) \longrightarrow \Gamma_{2}^{2}\left(\Gamma_{2}(A \oplus A) \rightarrow A \otimes A\right)
$$


is an epimorphism. By the naturality of $\theta_{A}$, we have $\theta_{A}=0$ because $\theta_{A_{0}}=0$. Now, from diagram (3-4), there is a commutative diagram of natural short exact sequences

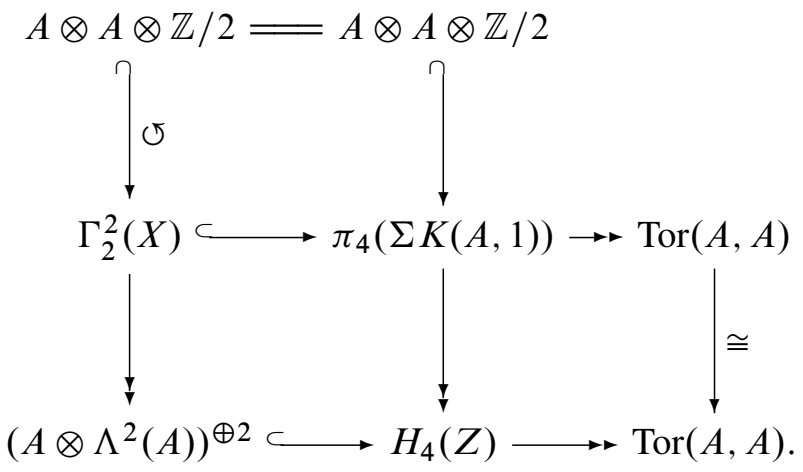

It follows that there is a natural (on $A$ ) isomorphism

$$
\Gamma_{2}^{2}(X) \cong A \otimes A \otimes \mathbb{Z} / 2 \oplus\left(A \otimes \Lambda^{2}(A)\right)^{\oplus 2} .
$$

Since $\left(A \otimes \Lambda^{2}(A)\right)^{\oplus 2}$ is an (unnatural) summand of $H_{4}(Z)$, it is an (unnatural) summand of $\pi_{4}(\Sigma K(A, 1))$. The proof is finished.

Corollary 3.5 Let $p$ be an odd prime integer. Then

$$
\pi_{4}\left(\Sigma K\left(\mathbb{Z} / p^{r}, 1\right)\right)=\mathbb{Z} / p^{r}
$$

and the Hurewicz homomorphism

$$
\pi_{4}\left(\Sigma K\left(\mathbb{Z} / p^{r}, 1\right)\right) \rightarrow H_{4}\left(\Sigma K\left(\mathbb{Z} / p^{r}, 1\right)\right)
$$

is an isomorphism.

Proof In this case, $A \otimes A \otimes \mathbb{Z} / 2=0$. Since $\mathbb{Z} / p^{r}$ is cyclic, $\Lambda^{2}(A) \otimes A=0$ and hence the result follows.

For completely determining the group $\pi_{4}(\Sigma K(A, 1))$, we have to consider the divisibility problem of the elements in the subgroup $A \otimes A \otimes \mathbb{Z} / 2=\Gamma_{4}(Z) \subseteq \pi_{4}(\Sigma K(A, 1))=$ $\pi_{4}(Z)$. We solve this problem for any finitely generated abelian group $A$.

Lemma 3.6 Let $A$ be any abelian group and let $j: M(A, 1) \rightarrow K(A, 1)$ be a map such that $j_{*}: H_{1}(M(A, 1)) \rightarrow H_{1}(K(A, 1))$ is an isomorphism. Then there is an (unnatural) splitting exact sequence

$$
\pi_{4}(\Sigma M(A, 1) \wedge M(A, 1)) \stackrel{\curvearrowleft}{\leftrightarrows}(\Sigma j \wedge j)_{*} \pi_{4}(\Sigma K(A, 1) \wedge K(A, 1)) \stackrel{\curvearrowleft}{\rightarrow}\left(A \otimes \Lambda^{2}(A)\right)^{\oplus 2} .
$$


Proof Let $X=\Sigma M(A, 1) \wedge M(A, 1)$ and let $Z=\Sigma K(A, 1) \wedge K(A, 1)$. The assertion follows from the commutative diagram of short exact sequences

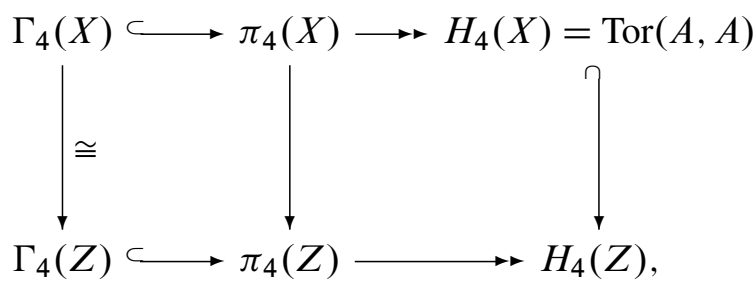

where the bottom row is short exact by Equation (3-5).

Given a finitely generated abelian group $A$, let

$$
A=A_{1} \oplus \bigoplus_{\substack{r \geq 1 \\ p \text { is a prime }}} A_{p^{r}}
$$

be the primary decomposition of $A$, where $A_{1}$ is torsion free and $A_{p^{r}}$ is a free $\mathbb{Z} / p^{r}$-module.

Theorem 3.7 Let $A$ be any finitely generated abelian group. Let $A=A_{2} \oplus B$ with $B=A_{1} \oplus \bigoplus_{p^{r} \neq 2} A_{p^{r}}$. Then

$\pi_{4}(\Sigma K(A, 1)) \cong \frac{1}{2}\left(A_{2} \otimes A_{2}\right) \oplus\left(A_{2} \otimes B\right)^{\oplus 2} \oplus B^{\otimes 2} \otimes \mathbb{Z} / 2 \oplus\left(A \otimes \Lambda^{2}(A)\right)^{\oplus 2}$ $\oplus \operatorname{Tor}\left(A_{2}, B\right)^{\oplus 2} \oplus \operatorname{Tor}(B, B)$,

where $\frac{1}{2}\left(A_{2} \otimes A_{2}\right)$ is a free $\mathbb{Z} / 4$-module with rank of $\operatorname{dim}_{\mathbb{Z} / 2}\left(A_{2} \otimes A_{2}\right)$.

Proof Let $X=\Sigma M(A, 1) \wedge M(A, 1)$. By Lemma 3.6, it suffices to show that $\pi_{4}(X) \cong \frac{1}{2}\left(A_{2} \otimes A_{2}\right) \oplus\left(A_{2} \otimes B\right)^{\oplus 2} \oplus B^{\otimes 2} \otimes \mathbb{Z} / 2 \oplus \operatorname{Tor}\left(A_{2}, B\right)^{\oplus 2} \oplus \operatorname{Tor}(B, B)$.

Observe that there is a homotopy decomposition

$$
X \simeq \bigvee_{\substack{r, s \geq 0 \\ p, q \text { prime }}} \sum M\left(A_{p^{r}}, 1\right) \wedge M\left(A_{q^{s}}, 1\right),
$$

where we allow $r, s$ to be 0 for having the factor $A_{1}$ to be appeared. Thus there is a decomposition

$$
\pi_{4}(X) \cong \bigoplus_{\substack{r, s \geq 0 \\ p, q \text { prime }}} \pi_{4}\left(\Sigma M\left(A_{p^{r}}, 1\right) \wedge M\left(A_{q^{s}}, 1\right)\right) .
$$


Let $r, s \geq 1$ and let $p$ and $q$ be positive prime integers. From [12, Corollary 6.6], there is a homotopy decomposition

$$
\begin{aligned}
& \sum M\left(\mathbb{Z} / p^{r}, 1\right) \wedge M\left(\mathbb{Z} / q^{s}, 1\right) \\
\simeq & \begin{cases}* & \text { if } p \neq q, \\
M\left(\mathbb{Z} / p^{\min \{r, s\}}, 3\right) \vee M\left(\mathbb{Z} / p^{\min \{r, s\}}, 4\right) & \text { if } p=q \text { and } \max \left\{p^{r}, q^{s}\right\}>2 .\end{cases}
\end{aligned}
$$

By taking $\pi_{4}$ to above decomposition, we have

(3-8) $\pi_{4}\left(\Sigma M\left(\mathbb{Z} / p^{r}, 1\right) \wedge M\left(\mathbb{Z} / q^{s}, 1\right)\right) \cong \mathbb{Z} / p^{r} \otimes \mathbb{Z} / q^{s} \otimes \mathbb{Z} / 2 \oplus \operatorname{Tor}\left(\mathbb{Z} / 2^{r}, \mathbb{Z} / 2^{s}\right)$

if $\max \left\{p^{r}, q^{s}\right\}>2$. Clearly this formula also holds for the case where $p^{r}=1$ or $q^{s}=1$. For the case $p^{r}=q^{s}=2$, we claim that

$$
\left.\pi_{4}(\Sigma M(\mathbb{Z} / 2,1) \wedge M(\mathbb{Z} / 2,1))\right)=\mathbb{Z} / 4 .
$$

Let $Y=\Sigma M(\mathbb{Z} / 2,1) \wedge M(\mathbb{Z} / 2,1)$. From the short exact sequence

$$
\Gamma_{4}(Y)=\mathbb{Z} / 2 \longrightarrow \pi_{4}(Y) \longrightarrow H_{4}(Y)=\mathbb{Z} / 2,
$$

the group $\pi_{4}(Y)=\mathbb{Z} / 4$ or $\mathbb{Z} / 2 \oplus \mathbb{Z} / 2$. Suppose that $\pi_{4}(Y)=\mathbb{Z} / 2 \oplus \mathbb{Z} / 2$. Then there exists an element $\alpha \in \pi_{4}(Y)$ of order 2 which has the nontrivial Hurewicz image. Since $\alpha$ is of order 2, the map $\alpha: S^{4} \rightarrow Y$ extends to a map $\tilde{\alpha}: M(\mathbb{Z} / 2,4) \rightarrow Y$ with

$$
\tilde{\alpha}_{*}: H_{4}(M(\mathbb{Z} / 2,4)) \longrightarrow H_{4}(Y)
$$

an isomorphism. Let

$$
j: M(\mathbb{Z} / 2,3) \longrightarrow Y
$$

be the canonical inclusion. Then $j_{*}: H_{3}(M(\mathbb{Z} / 2,3)) \rightarrow H_{3}(Y)$ is an isomorphism. Then

$$
(j, \widetilde{\alpha}): M(\mathbb{Z} / 2,3) \vee M(\mathbb{Z} / 2,4) \longrightarrow Y
$$

is a homotopy equivalence because it induces an isomorphism on homology, which contradicts that the Steenrod operation $\mathrm{Sq}^{2}: H^{3}(Y ; \mathbb{Z} / 2) \rightarrow H^{5}(Y ; \mathbb{Z} / 2)$ is an isomorphism. Thus $\pi_{4}(Y)=\mathbb{Z} / 4$.

Now the assertion follows from decomposition (3-6) and the computational formulae (3-8) and (3-9).

Corollary 3.8 Let $A_{2}$ be any elementary 2-group. Then there is a natural short exact sequence

$$
\left(A_{2} \otimes \Lambda^{2}\left(A_{2}\right)\right)^{\oplus 2} \longrightarrow \pi_{4}\left(\Sigma K\left(A_{2}, 1\right)\right) \longrightarrow \frac{1}{2}\left(A_{2} \otimes A_{2}\right),
$$

where $\frac{1}{2}\left(A_{2} \otimes A_{2}\right)$ is a free $\mathbb{Z} / 4-$ module. Moreover this splits off unnaturally. 
Proof By Theorem 3.4, there is a natural short exact sequence $\left(A_{2} \otimes \Lambda^{2}\left(A_{2}\right)\right)^{\oplus 2} \longrightarrow \pi_{4}\left(\Sigma K\left(A_{2}, 1\right)\right) \longrightarrow \pi_{4}\left(\Sigma K\left(A_{2}, 1\right)\right) /\left(A_{2} \otimes \Lambda^{2}\left(A_{2}\right)\right)^{\oplus 2}$. By Theorem 3.7, the quotient group $\pi_{4}\left(\Sigma K\left(A_{2}, 1\right)\right) /\left(A_{2} \otimes \Lambda^{2}\left(A_{2}\right)\right)^{\oplus 2}$ is free $\mathbb{Z} / 4-$ module for any finite dimensional elementary 2 -groups. The assertion follows by taking direct limits.

Remark 3.9 The summand $\frac{1}{2}\left(A_{2} \otimes A_{2}\right)$ is subquotient functor of $\pi_{4}(\Sigma K(A, 1))$ on $A$ in the following sense. For any abelian group $A$, the $\mathbb{Z} / 2$-component $A_{2}$ is given by the image of

$$
\mathrm{Sq}_{*}^{1}: H_{2}(A ; \mathbb{Z} / 2) \longrightarrow H_{1}(A ; \mathbb{Z} / 2)
$$

Thus $A \mapsto A_{2}$ is a subfunctor of the identity functor on abelian groups. Then $\pi_{4}\left(\Sigma K\left(A_{2}, 1\right)\right)$ is a subfunctor of $\pi_{4}(\Sigma K(A, 1))$ on $A$ and so

$$
\frac{1}{2}\left(A_{2} \otimes A_{2}\right)=\pi_{4}\left(\Sigma K\left(A_{2}, 1\right)\right) /\left(A_{2} \otimes \Lambda^{2}\left(A_{2}\right)\right)^{\oplus 2}
$$

is a subquotient functor of $\pi_{4}(\Sigma K(A, 1))$ on $A$.

\subsection{Applications}

As an application, we compute $\pi_{i}\left(M\left(\mathbb{Z} / p^{r}, 2\right)\right)$ for $i \leq 4$. By the Hurewicz Theorem, $\pi_{2}\left(M\left(\mathbb{Z} / p^{r}, 2\right)\right)=\mathbb{Z} / p^{r}$. From the Whitehead exact sequence (2-2), we have

$$
\Gamma_{n}\left(M\left(\mathbb{Z} / p^{r}, 2\right)\right)=\pi_{n}\left(M\left(\mathbb{Z} / p^{r}, 2\right)\right)
$$

for $r \geq 3$ because $H_{i}\left(M\left(\mathbb{Z} / p^{r}, 2\right)=0\right.$ for $i \geq 3$. It follows directly that

$$
\begin{aligned}
\pi_{3}\left(M\left(\mathbb{Z} / p^{r}, 2\right)\right) & =\Gamma_{3}\left(M\left(\mathbb{Z} / p^{r}, 2\right)\right) \\
& =\Gamma_{2}\left(\mathbb{Z} / p^{r}\right)= \begin{cases}\mathbb{Z} / p^{r} & \text { if } p>2, \\
\mathbb{Z} / 2^{r+1} & \text { if } p=2,\end{cases}
\end{aligned}
$$

where $\Gamma_{2}(A)$ is computed in Example 2.2. From Theorem 2.5 (1), there is a short exact sequence

$\Gamma_{2}^{2}\left(M\left(\mathbb{Z} / p^{r}, 2\right)\right) \hookrightarrow \Gamma_{4}\left(M\left(\mathbb{Z} / p^{r}, 2\right)\right) \longrightarrow R_{2}\left(\pi_{2}\left(M\left(\mathbb{Z} / p^{r}, 2\right)\right)\right)=R_{2}\left(\mathbb{Z} / p^{r}\right)$.

From Section 2.2,

$$
R_{2}\left(\mathbb{Z} / p^{r}\right)=\left\{\begin{array}{lll}
0 & \text { if } p>2 \\
\mathbb{Z} / 2 & \text { if } p=2
\end{array}\right.
$$


By the definition (2-4) of the functor $\Gamma_{2}^{2}$, the group $\Gamma_{2}^{2}\left(M\left(\mathbb{Z} / p^{r}, 2\right)\right)$ is given by the pushout

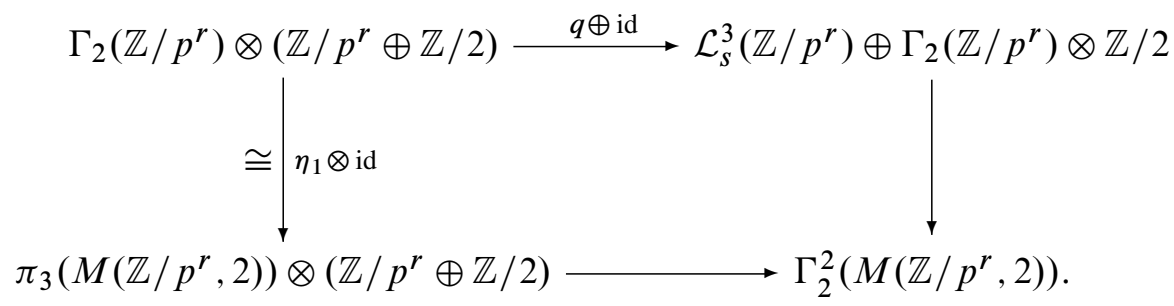

Thus

$$
\Gamma_{2}^{2}\left(M\left(\mathbb{Z} / p^{r}, 2\right)\right) \cong \mathcal{L}_{s}^{3}\left(\mathbb{Z} / p^{r}\right) \oplus \Gamma_{2}\left(\mathbb{Z} / p^{r}\right) \otimes \mathbb{Z} / 2 .
$$

Since $\mathbb{Z} / p^{r}$ is cyclic and $\mathcal{L}_{S}^{3}(A)$ is isomorphic to the kernel of $A \otimes \Lambda^{2}(A) \rightarrow \Lambda^{3}(A)$, we have

$$
\mathcal{L}_{S}^{3}\left(\mathbb{Z} / p^{r}\right)=0
$$

and so

$$
\Gamma_{2}^{2}\left(M\left(\mathbb{Z} / p^{r}, 2\right)\right)=\left\{\begin{aligned}
0 & \text { if } p>2 \\
\mathbb{Z} / 2 & \text { if } p=2
\end{aligned}\right.
$$

A direct consequence is

$$
\pi_{4}\left(\Sigma M\left(\mathbb{Z} / p^{r}, 2\right)\right)=0 \text { for } p>2 .
$$

For the case $p=2$, we have the short exact sequence

$$
\mathbb{Z} / 2 \subset \pi_{4}\left(M\left(\mathbb{Z} / 2^{r}, 2\right)\right) \longrightarrow \mathbb{Z} / 2 \text {. }
$$

The remaining problem is to decide whether $\pi_{4}\left(M\left(\mathbb{Z} / 2^{r}, 2\right)\right)$ is equal to $\mathbb{Z} / 2 \oplus \mathbb{Z} / 2$ or $\mathbb{Z} / 4$. It has been computed in [21] that $\pi_{4}(M(\mathbb{Z} / 2,2))=\mathbb{Z} / 4$. For $r>1$, the group $\pi_{4}\left(M\left(\mathbb{Z} / 2^{r}, 2\right)\right)$ seems not recorded in references. We are going to determine the group $\pi_{4}\left(M\left(\mathbb{Z} / 2^{r}, 2\right)\right)$ using our methods.

\section{Lemma 3.10 Let}

$$
j: M\left(\mathbb{Z} / 2^{r}, 2\right) \longrightarrow \Sigma K\left(\mathbb{Z} / 2^{r}, 1\right)
$$

be the canonical map inducing isomorphism on $\mathrm{H}_{2}$. Then

$$
j_{*}: \Gamma_{4}\left(M\left(\mathbb{Z} / 2^{r}, 2\right)\right) \longrightarrow \Gamma_{4}\left(\Sigma K\left(\mathbb{Z} / 2^{r}, 1\right)\right)
$$

is an isomorphism. 
Proof By Theorem 2.5(1), there is a commutative diagram of short exact sequences

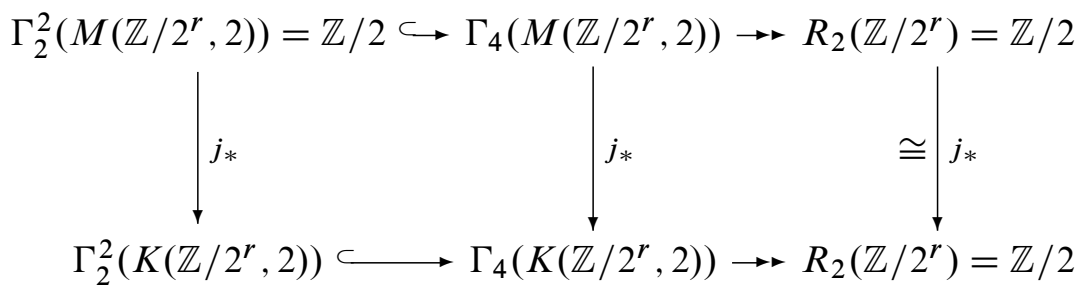

From the Whitehead exact sequence

$$
\Gamma_{3}\left(\mathbb{Z} / 2^{r}\right)=\mathbb{Z} / 2^{r+1} \rightarrow \pi_{3}\left(\Sigma K\left(\mathbb{Z} / 2^{r}, 1\right)\right)=\mathbb{Z} / 2^{r} \otimes \mathbb{Z} / 2^{r}=\mathbb{Z} / 2^{r} \rightarrow 0,
$$

we have

$\eta_{1} \otimes$ id: $\Gamma_{2}\left(\mathbb{Z} / 2^{r}\right) \otimes\left(\mathbb{Z} / 2^{r} \oplus \mathbb{Z} / 2\right) \longrightarrow \pi_{3}\left(K\left(\mathbb{Z} / 2^{r}, 2\right)\right) \otimes\left(\mathbb{Z} / 2^{r} \oplus \mathbb{Z} / 2\right)$ is an isomorphism. Similar to the computation of $\Gamma_{2}^{2}\left(M\left(\mathbb{Z} / 2^{r}, 2\right)\right)$, we have

$$
\Gamma_{2}^{2}\left(K\left(\mathbb{Z} / 2^{r}, 2\right)\right)=\mathbb{Z} / 2
$$

with an isomorphism $j_{*}: \Gamma_{2}^{2}\left(M\left(\mathbb{Z} / 2^{r}, 2\right)\right) \cong \Gamma_{2}^{2}\left(K\left(\mathbb{Z} / 2^{r}, 2\right)\right)$. The assertion then follows by 5 -lemma.

\section{Lemma 3.11 The group}

$$
\Gamma_{4}\left(\Sigma K\left(\mathbb{Z} / 2^{r}, 1\right)\right)= \begin{cases}\mathbb{Z} / 2 \oplus \mathbb{Z} / 2 & \text { if } r>1 \\ \mathbb{Z} / 4 & \text { if } r=1\end{cases}
$$

Proof Let $Z=\Sigma K\left(\mathbb{Z} / 2^{r}, 1\right) \wedge K\left(\mathbb{Z} / 2^{r}, 1\right)$ and let $H: Z \rightarrow \Sigma K\left(\mathbb{Z} / 2^{r}, 1\right)$ be the Hopf map. From Equation (3-5), there is a commutative diagram of exact sequence

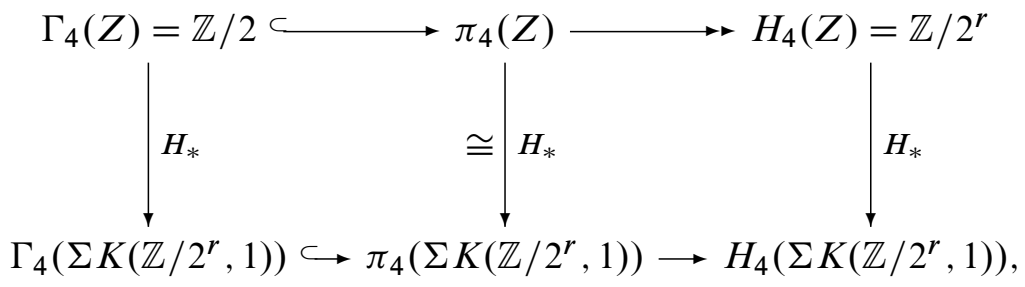

where the bottom row is left exact because $H_{5}\left(\Sigma K\left(\mathbb{Z} / 2^{r}, 1\right)\right)=H_{4}\left(\mathbb{Z} / 2^{r}\right)=0$.

If $r>1$, then $\Gamma_{4}(Z)$ is a summand of $\pi_{4}(Z) \cong \pi_{4}\left(\Sigma K\left(\mathbb{Z} / 2^{r}, 1\right)\right)$ by Theorem 3.7. Thus $\Gamma_{4}(Z)=\mathbb{Z} / 2$ is also a summand of $\Gamma_{4}\left(\Sigma K\left(\mathbb{Z} / 2^{r}, 1\right)\right)$. It follows that

$$
\Gamma_{4}\left(\Sigma K\left(\mathbb{Z} / 2^{r}, 1\right)\right)=\mathbb{Z} / 2 \oplus \mathbb{Z} / 2 \quad \text { if } r>1 .
$$


If $r=1$, by Corollary $3.8, \pi_{4}(\Sigma K(\mathbb{Z} / 2,1))=\mathbb{Z} / 4$ and so

$$
\Gamma_{4}(\Sigma K(\mathbb{Z} / 2,1)) \cong \pi_{4}(\Sigma K(\mathbb{Z} / 2,1))=\mathbb{Z} / 4 .
$$

The proof is finished.

Since $\Gamma_{4}\left(\Sigma K\left(\mathbb{Z} / 2^{r}, 1\right)\right) \rightarrow \pi_{4}\left(\Sigma K\left(\mathbb{Z} / 2^{r}, 1\right)\right)$ is a monomorphism, from Lemmas 3.10 and 3.11, we have the following:

\section{Corollary 3.12 Let}

$$
j: M\left(\mathbb{Z} / 2^{r}, 2\right) \longrightarrow \Sigma K\left(\mathbb{Z} / 2^{r}, 1\right)
$$

be the canonical map inducing isomorphism on $\mathrm{H}_{2}$. Then

(1) $\pi_{4}(M(\mathbb{Z} / 2,2))=\mathbb{Z} / 4$ and

$$
j_{*}: \pi_{4}(M(\mathbb{Z} / 2,2)) \rightarrow \pi_{4}(\Sigma K(\mathbb{Z} / 2,1))
$$

is an isomorphism;

(2) For $r>1, \pi_{4}\left(M\left(\mathbb{Z} / 2^{r}, 2\right)\right)=\mathbb{Z} / 2 \oplus \mathbb{Z} / 2$ and

$j_{*}: \pi_{4}\left(M\left(\mathbb{Z} / 2^{r}, 2\right)\right)=\mathbb{Z} / 2 \oplus \mathbb{Z} / 2 \rightarrow \pi_{4}\left(\Sigma K\left(\mathbb{Z} / 2^{r}, 1\right)\right)=\mathbb{Z} / 2 \oplus \mathbb{Z} / 2^{r}$

is a monomorphism.

Note that $M(\mathbb{Z} / 2,2)=\Sigma \mathbb{R} \mathrm{P}^{2}$ and $\Sigma K(\mathbb{Z} / 2,1)=\Sigma \mathbb{R} \mathrm{P}^{\infty}$ with the canonical inclusion $j: \Sigma \mathbb{R} \mathrm{P}^{2} \hookrightarrow \Sigma \mathbb{R} \mathrm{P}^{\infty}$. A consequence of Corollary 3.12 (1) on the suspended projective spaces are as follows.

Corollary 3.13 Let $j: \Sigma \mathbb{R} \mathrm{P}^{2} \rightarrow \Sigma \mathbb{R} \mathrm{P}^{n}$ be the canonical inclusion with $3 \leq n \leq \infty$.

(1) For $4 \leq n \leq \infty, j_{*}: \pi_{4}\left(\Sigma \mathbb{R} \mathrm{P}^{2}\right)=\mathbb{Z} / 4 \rightarrow \pi_{4}\left(\Sigma \mathbb{R} \mathrm{P}^{n}\right)$ is an isomorphism.

(2) For $n=3, j_{*}: \pi_{4}\left(\Sigma \mathbb{R} \mathrm{P}^{2}\right)=\mathbb{Z} / 4 \rightarrow \pi_{4}\left(\Sigma \mathbb{R}^{3}\right)$ is a splitting monomorphism. Moreover

$$
\pi_{4}\left(\Sigma \mathbb{R} \mathrm{P}^{3}\right) \cong \pi_{4}\left(\Sigma \mathbb{R} \mathrm{P}^{2}\right) \oplus \mathbb{Z}=\mathbb{Z} / 4 \oplus \mathbb{Z}
$$

Proof Assertion (1) and the first part of assertion (2) are direct consequences of Corollary 3.12. For the second part of assertion (2), notice that $\mathbb{R} \mathrm{P}^{3}=\mathrm{SO}(3)$. From the commutative diagram

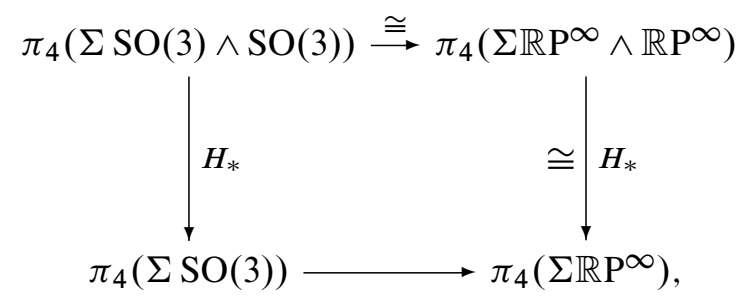


we have

$$
\begin{aligned}
\pi_{4}(\Sigma \mathrm{SO}(3)) & \cong \pi_{4}(\Sigma \mathrm{SO}(3) \wedge \mathrm{SO}(3)) \oplus \pi_{4}(B \mathrm{SO}(3)) \\
& \cong \pi_{4}\left(\Sigma \mathbb{R} \mathrm{P}^{\infty}\right) \oplus \pi_{3}(\mathrm{SO}(3)) \\
& \cong \mathbb{Z} / 4 \oplus \mathbb{Z}
\end{aligned}
$$

and hence the result follows.

Another consequence is as follows:

Corollary 3.14 Let $\Sigma_{3}$ be the third symmetric group. Then $\pi_{4}\left(\Sigma K\left(\Sigma_{3}, 1\right)\right)=\mathbb{Z} / 12$.

Proof Recall that the integral homology groups of $\Sigma_{3}$ are 4-periodic with the following initial terms:

$$
H_{1}\left(\Sigma_{3}\right)=\mathbb{Z} / 2, H_{2}\left(\Sigma_{3}\right)=0, H_{3}\left(\Sigma_{3}\right)=\mathbb{Z} / 6, H_{4}\left(\Sigma_{3}\right)=0 .
$$

Let $X=\Sigma K\left(\Sigma_{3}, 1\right)$. The Whitehead exact sequence has the following form:

$\Gamma_{4}(X) \subset \pi_{4}(X) \longrightarrow H_{3}\left(\Sigma_{3}\right)=\mathbb{Z} / 6 \longrightarrow \Gamma_{3}(X)=\mathbb{Z} / 4 \longrightarrow \pi_{3}(X)=\mathbb{Z} / 2$.

The inclusion $\Sigma_{2}=\mathbb{Z} / 2 \rightarrow \Sigma_{3}$ induces an isomorphism

$$
\pi_{i}(\Sigma K(\mathbb{Z} / 2,1))=\mathbb{Z} / 2 \stackrel{\cong}{\longrightarrow} \pi_{i}\left(\Sigma K\left(\Sigma_{3}, 1\right)\right)=\mathbb{Z} / 2
$$

for $i=2,3$. By Theorem 2.5 (1) together with Lemma 3.11, the inclusion $\Sigma_{2}=$ $\mathbb{Z} / 2 \rightarrow \Sigma_{3}$ induces an isomorphism

$$
\Gamma_{4}(\Sigma K(\mathbb{Z} / 2,1))=\mathbb{Z} / 4 \stackrel{\cong}{\longrightarrow} \Gamma_{4}\left(\Sigma K\left(\Sigma_{3}, 1\right)\right)
$$

and hence the result holds.

\section{On group $\pi_{5}(\Sigma K(A, 1))$}

\subsection{Some properties of the functor $A \mapsto \pi_{5}(\Sigma K(A, 1))$}

From Hopf fibration

$$
\Sigma K(A, 1) \wedge K(A, 1) \longrightarrow \Sigma K(A, 1) \longrightarrow K(A, 2)
$$


it suffices to compute $\pi_{5}(\Sigma K(A, 1) \wedge K(A, 1))$. Let $Z=\Sigma K(A, 1) \wedge K(A, 1)$. Since $Z$ is 2 -connected, from Theorem 2.5(2), there are natural exact sequences

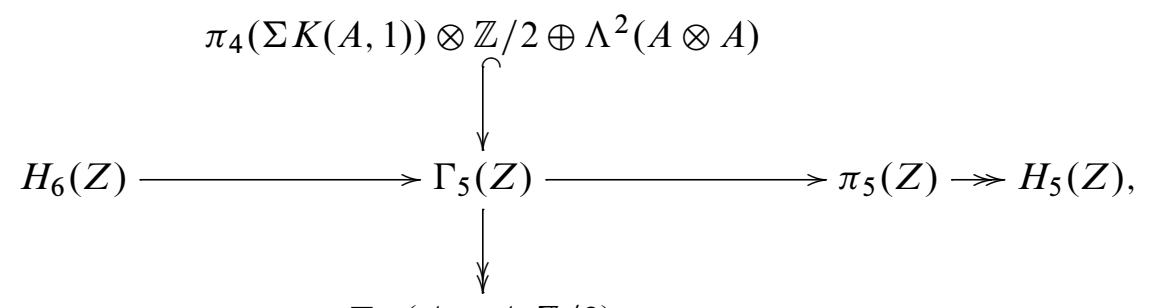

\section{$\operatorname{Tor}(A \otimes A, \mathbb{Z} / 2)$}

where $\pi_{5}(Z) \rightarrow H_{5}(Z)$ is onto by Equation (3-5). The group $\pi_{4}(\Sigma K(A, 1))$ has been determined by Theorems 3.4 and 3.7.

Proposition 4.1 Let $A$ be a free abelian group. Then there is a natural short exact sequence

$\left(\Lambda^{2}(A) \otimes A \otimes \mathbb{Z} / 2\right)^{\oplus 2} \oplus A^{\otimes 2} \otimes \mathbb{Z} / 2 \oplus \Lambda^{2}(A \otimes A)$

$$
\hookrightarrow \pi_{5}(\Sigma K(A, 1)) \rightarrow\left(\Lambda^{3}(A) \otimes A\right)^{\oplus 2} \oplus \Lambda^{2}(A)^{\otimes 2} .
$$

Proof Since $A$ is a free abelian group, the Hurewicz homomorphism $h_{*}: \pi_{*}(Z) \rightarrow$ $\widetilde{H}_{*}(Z)$ is onto because $Z$ is a wedge of spheres. Thus there is a short exact sequence

$$
\Gamma_{5}(Z) \subset \pi_{5}(Z) \longrightarrow H_{5}(Z) .
$$

By Theorem 3.4, $\pi_{4}(Z) \cong\left(\Lambda^{2}(A) \otimes A\right)^{\oplus 2} \oplus A \otimes A \otimes \mathbb{Z} / 2$ for a free abelian group $A$. The assertion follows from diagram (4-1).

Proposition 4.2 If $A$ is a torsion abelian group with the property that 2: $A \rightarrow A$ is an isomorphism, then there is a natural short exact sequence

$$
\Lambda^{2}(A \otimes A) \smile \pi_{5}(\Sigma K(A, 1)) \longrightarrow H_{4}(K(A, 1) \wedge K(A, 1)) .
$$

Proof It suffices to show that the Hurewicz homomorphism $h_{*}: \pi_{6}(Z) \rightarrow H_{6}(Z)$ is onto. We may assume that $A$ is finitely generated because we can take direct limit for general case whence the finitely generated case is proved. Then $A$ is a direct sum of the primary $p$-torsion groups $\mathbb{Z} / p^{r}$ for some $r \geq 1$ and odd primes $p$. According to [11], there is homotopy decomposition

$$
\Sigma K\left(\mathbb{Z} / p^{r}, 1\right) \simeq X_{1} \vee \cdots \vee X_{p-1},
$$

where $\bar{H}_{q}\left(X_{i} ; \mathbb{Z}\right) \neq 0$ if and only if $q \equiv 2 i \bmod 2 p-2$. Together with the decomposition formula (3-7) for the smash product of Moore spaces, up to 6-skeleton, 
$\Sigma K(A, 1) \wedge K(A, 1)$ is homotopy equivalent to a wedge of spheres and Moore spaces. It follows that the Hurewicz homomorphism

$$
\pi_{6}(\Sigma K(A, 1) \wedge K(A, 1)) \longrightarrow H_{6}(\Sigma K(A, 1) \wedge K(A, 1))
$$

is onto and hence the result holds.

From the above proof, we also have the following:

Proposition 4.3 Let $A$ be any abelian group. Let $\mathbb{Z}_{1 / 2}=\left\{m / 2^{r} \in \mathbb{Q} \mid m \in \mathbb{Z}, r \geq 0\right\}$. Then there is natural short exact sequence

$\Lambda^{2}(A \otimes A) \otimes \mathbb{Z}_{1 / 2} \longrightarrow \pi_{5}(\Sigma K(A, 1)) \otimes \mathbb{Z}_{1 / 2}$

$$
\longrightarrow H_{4}(K(A, 1) \wedge K(A, 1)) \otimes \mathbb{Z}_{1 / 2} \text {. }
$$

For computing the group $\pi_{5}(\Sigma K(A, 1))$, as one see from the above, the tricky part is the 2-torsion. Whence $A$ contains 2 -torsion summands, the Hurewicz homomorphism $\pi_{6}(Z) \rightarrow H_{6}(Z)$ is no longer epimorphism in general and so $\Gamma_{5}(Z) \rightarrow \pi_{5}(Z)$ is not a monomorphism in general. Also the group $\pi_{5}(Z)$ in diagram (4-1) admits nontrivial extension. The computation of the group $\pi_{5}(\Sigma K(A, 1))$ for finitely generated abelian groups $A$ can be given by the following steps:

Step 1 Take a primary decomposition of $A$ and write $K(A, 1)$ as a product of copies of $S^{1}=K(\mathbb{Z}, 1)$ and $K\left(\mathbb{Z} / p^{r}, 1\right)$.

Step 2 By using the fact that $\Sigma X \times Y \simeq \Sigma X \vee \Sigma Y \simeq \Sigma X \wedge Y$ for any spaces $X$ and $Y$, one gets

$$
\begin{aligned}
\Sigma\left(X_{1} \times X_{2}\right) \wedge\left(X_{1} \times X_{2}\right) \simeq & \Sigma\left(X_{1} \vee X_{2} \vee X_{1} \wedge X_{2}\right) \wedge\left(X_{1} \vee X_{2} \vee X_{1} \wedge X_{2}\right) \\
\simeq & \Sigma\left(X_{1}^{\wedge 2} \vee X_{2}^{\wedge 2} \vee X_{1}^{\wedge 2} \wedge X_{2}^{\wedge 2}\right. \\
& \left.\vee \bigvee^{2} X_{1} \wedge X_{2} \vee \bigvee^{2} X_{1}^{\wedge 2} \wedge X_{2} \vee \stackrel{2}{\bigvee} X_{1} \wedge X_{2}^{\wedge 2}\right)
\end{aligned}
$$

From this, $\Sigma K(A, 1) \wedge K(A, 1)$ is then homotopy equivalent to a wedge of the spaces in the form

$$
X=\Sigma^{m} K\left(\mathbb{Z} / p_{1}^{r_{1}}, 1\right) \wedge K\left(\mathbb{Z} / p_{2}^{r_{2}}, 1\right) \wedge \cdots \wedge K\left(\mathbb{Z} / p_{t}^{r_{t}}, 1\right)
$$

with $m+t \geq 3$ and $m \geq 1$. 
Step 3 By applying the Hilton-Milnor Theorem, we have

$$
\begin{aligned}
\Omega(\Sigma X \vee \Sigma Y) & \simeq \Omega \Sigma X \times \Omega \Sigma Y \times \Omega \Sigma((\Omega \Sigma X) \wedge(\Omega \Sigma Y)) \\
& \simeq \Omega \Sigma X \times \Omega \Sigma Y \times \Omega \Sigma\left(\bigvee_{i, j=1}^{\infty} X^{\wedge i} \wedge Y^{\wedge j}\right) \\
\pi_{n}(\Sigma X \vee \Sigma Y) & \cong \pi_{n}(\Sigma X) \oplus \pi_{n}(\Sigma Y) \oplus \pi_{n}\left(\bigvee_{i, j=1}^{\infty} \Sigma X^{\wedge i} \wedge Y^{\wedge j}\right)
\end{aligned}
$$

Thus

Note that the connectivity of $X^{\wedge i} \wedge Y^{\wedge j}$ tends to $\infty$ as $i, j \rightarrow \infty$. By repeating the above procedure, $\pi_{n}(\Sigma K(A, 1) \wedge K(A, 1))$ is isomorphism to a direct sum of the groups $\pi_{n}(X)$ with $X$ given in the form above.

Notice that

$$
\Sigma^{m} K\left(\mathbb{Z} / p_{1}^{r_{1}}, 1\right) \wedge K\left(\mathbb{Z} / p_{2}^{r_{2}}, 1\right) \wedge \cdots \wedge K\left(\mathbb{Z} / p_{t}^{r_{t}}, 1\right) \simeq *
$$

if the primes $p_{i} \neq p_{j}$ for some $i \neq j$. Thus we only need to compute

$$
\pi_{5}\left(\Sigma^{m} K\left(\mathbb{Z} / p^{r_{1}}, 1\right) \wedge K\left(\mathbb{Z} / p^{r_{2}}, 1\right) \wedge \cdots \wedge K\left(\mathbb{Z} / p^{r_{t}}, 1\right)\right)
$$

for a prime $p$. If $t=0$, the homotopy group $\pi_{5}\left(S^{m}\right)$ is known by $\pi_{5}\left(S^{3}\right)=$ $\pi_{5}\left(S^{4}\right)=\mathbb{Z} / 2$ and $\pi_{5}\left(S^{5}\right)=\mathbb{Z}$. For an odd prime $p$, this homotopy group can be determined by Proposition 4.2. The rest work in this section is of course to compute $\pi_{5}\left(\Sigma^{m} K\left(\mathbb{Z} / 2^{r_{1}}, 1\right) \wedge K\left(\mathbb{Z} / 2^{r_{2}}, 1\right) \wedge \cdots \wedge K\left(\mathbb{Z} / 2^{r_{t}}, 1\right)\right)$ with $m+t \geq 3$. When $m+t \geq$ 5 , we have

$$
\pi_{5}(X)= \begin{cases}0 & \text { if } m+t>5 \\ \mathbb{Z} / 2^{\min \left\{r_{1}, \ldots, r_{t}\right\}} & \text { if } m+t=5 \text { with } t \geq 1\end{cases}
$$

for $X=\Sigma^{m} K\left(\mathbb{Z} / 2^{r_{1}}, 1\right) \wedge K\left(\mathbb{Z} / 2^{r_{2}}, 1\right) \wedge \cdots \wedge K\left(\mathbb{Z} / 2^{r_{t}}, 1\right)$. The first less obvious case is $m+t=4$, which will be discussed in the next subsection.

\subsection{The group $\pi_{5}\left(\Sigma^{m} K\left(\mathbb{Z} / 2^{r_{1}}, 1\right) \wedge K\left(\mathbb{Z} / 2^{r_{2}}, 1\right) \wedge \cdots \wedge K\left(\mathbb{Z} / 2^{r_{t}}, 1\right)\right)$ for $m+t=4$ and $m, t \geq 1$}

We first consider the case $t=1$.

Lemma 4.4 The Hurewicz homomorphism

$$
h_{5}: \pi_{5}\left(\Sigma^{2} K\left(\mathbb{Z} / 2^{r}, 1\right)\right) \rightarrow H_{5}\left(\Sigma^{2} K\left(\mathbb{Z} / 2^{r}, 1\right)\right)
$$

is onto for any $r \geq 1$. 
Proof Let $X=\Sigma^{2} K\left(\mathbb{Z} / 2^{r}, 1\right)$. Consider the Whitehead exact sequence

$$
\pi_{5}(X) \stackrel{h_{5}}{\longrightarrow} H_{5}(X) \rightarrow \Gamma_{4}(X)=\mathbb{Z} / 2 \rightarrow \pi_{4}(X) \rightarrow H_{4}(X)=0 .
$$

Thus the Hurewicz homomorphism $h_{5}$ is onto if and only if $\pi_{4}(X) \neq 0$.

Let $f: S^{3} \rightarrow X$ be a map representing the generator for $\pi_{3}(X)=\mathbb{Z} / 2^{r}$. From the remark to Theorem 2.4, $\pi_{4}(X)=0$ if and only if the composite

$$
S^{4} \stackrel{\eta}{\longrightarrow} S^{3} \stackrel{f}{\longrightarrow} X
$$

is null homotopic, if and only if the map $f: S^{3} \rightarrow X$ extends to a map $\tilde{f}: \Sigma \mathbb{C P}^{2} \rightarrow X$ because $\Sigma \mathbb{C P}^{2}$ is the homotopy cofibre of $\eta: S^{4} \rightarrow S^{3}$.

Suppose that there exists a map $\tilde{f}: \Sigma \mathbb{C} P^{2} \rightarrow X$ such that $\left.\tilde{f}\right|_{S^{4}}=f$. By taking mod 2 cohomology, there is commutative diagram

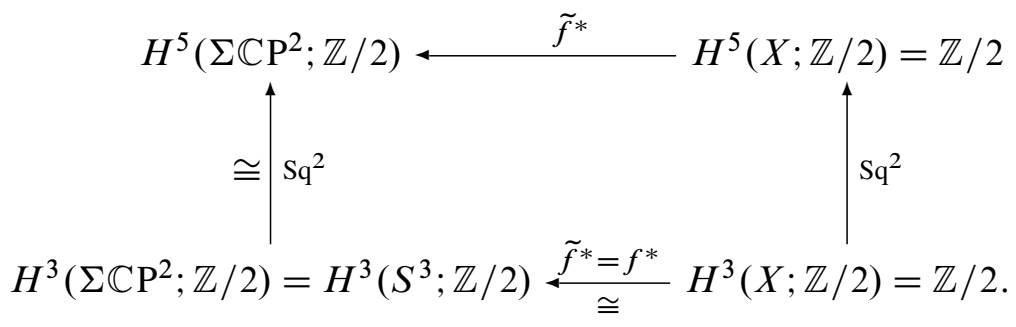

It follows that

$$
\mathrm{Sq}^{2}: H^{3}(X ; \mathbb{Z} / 2) \longrightarrow H^{5}(X ; \mathbb{Z} / 2)
$$

is an isomorphism. On the other hand, from the fact that $X=\Sigma^{2} K\left(\mathbb{Z} / 2^{r}, 1\right)$ and $\mathrm{Sq}^{2}: H^{1}\left(K\left(\mathbb{Z} / 2^{r}, 1\right) ; \mathbb{Z} / 2\right) \rightarrow H^{3}\left(K\left(\mathbb{Z} / 2^{r}, 1\right)\right)$ is zero, $\mathrm{Sq}^{2}: H^{3}(X ; \mathbb{Z} / 2) \rightarrow$ $H^{5}(X ; \mathbb{Z} / 2)$ is zero. This gives a contradiction. The assertion follows.

Proposition $4.5 \pi_{5}\left(\Sigma^{3} K\left(\mathbb{Z} / 2^{r}, 1\right)\right)=\mathbb{Z} / 2$ for $r \geq 1$.

Proof Let $X=\Sigma^{3} K\left(\mathbb{Z} / 2^{r}, 1\right)$. Consider the Whitehead exact sequence

$$
\pi_{6}(X) \stackrel{h_{6}}{\longrightarrow} H_{6}(X) \rightarrow \Gamma_{5}(X)=\mathbb{Z} / 2 \rightarrow \pi_{5}(X) \rightarrow H_{5}(X)=0 .
$$

By Lemma 4.4, $h_{6}: \pi_{6}(X) \rightarrow H_{6}(X)$ is onto. Thus $\pi_{5}(X) \cong \Gamma_{5}(X)=\mathbb{Z} / 2$.

Now we consider the case $t=2$.

Proposition 4.6 Let $r_{1}, r_{2} \geq 1$. Then

$$
\pi_{5}\left(\Sigma^{2} K\left(\mathbb{Z} / 2^{r_{1}}, 1\right) \wedge K\left(\mathbb{Z} / 2^{r_{2}}, 1\right)\right)= \begin{cases}\mathbb{Z} / 2 \oplus \mathbb{Z} / 2^{\min \left\{r_{1}, r_{2}\right\}} & \text { if } \max \left\{r_{1}, r_{2}\right\}>1, \\ \mathbb{Z} / 4 & \text { if } r_{1}=r_{2}=1 .\end{cases}
$$


Proof Let $X=\Sigma^{2} K\left(\mathbb{Z} / 2^{r_{1}}, 1\right) \wedge K\left(\mathbb{Z} / 2^{r_{2}}, 1\right)$. By Lemma 4.4, there exists a map

$$
f_{i}: S^{5} \longrightarrow \Sigma^{2} K\left(\mathbb{Z} / 2^{r_{i}}, 1\right), \quad i=1,2,
$$

which induces an epimorphism

$$
f_{i *}: H_{5}\left(S^{5}\right) \longrightarrow H_{5}\left(\Sigma^{2} K\left(\mathbb{Z} / 2^{r_{i}}, 1\right) .\right.
$$

Let $j: Y=\Sigma^{2} M\left(\mathbb{Z} / 2^{r_{1}}, 1\right) \wedge M\left(\mathbb{Z} / 2^{r_{2}}, 1\right) \hookrightarrow X$ be the canonical inclusion. Then the map

$$
f: Y \vee S^{5} \wedge K\left(\mathbb{Z} / 2^{r_{2}}, 1\right) \vee K\left(\mathbb{Z} / 2^{r_{1}}, 1\right) \wedge S^{5} \stackrel{\left(j, f_{1} \wedge \mathrm{id}, \mathrm{id} \wedge f_{2}\right)}{\longrightarrow} X
$$

induces an isomorphism on $H_{j}(; \mathbb{Z} / 2)$ for $j \leq 6$. Thus

$$
f_{*}: \pi_{k}\left(Y \vee S^{5} \wedge K\left(\mathbb{Z} / 2^{r_{2}}, 1\right) \vee K\left(\mathbb{Z} / 2^{r_{1}}, 1\right) \wedge S^{5}\right) \longrightarrow \pi_{k}(X)
$$

is an isomorphism for $k \leq 5$. Note that

$$
\pi_{k}\left(S^{5} \wedge K\left(\mathbb{Z} / 2^{r_{2}}, 1\right)\right)=\pi_{k}\left(K\left(\mathbb{Z} / 2^{r_{1}}, 1\right) \wedge S^{5}\right)=0
$$

for $k \leq 5$. Thus

$$
j_{*}: \pi_{k}(Y) \rightarrow \pi_{k}(X)
$$

is an isomorphism for $k \leq 5$. In particular, $\pi_{5}(Y) \cong \pi_{5}(X)$.

If max $r_{1}, r_{2}>1$, from decomposition (3-7), we have

$$
\Sigma^{2} M\left(\mathbb{Z} / 2^{r_{1}}, 1\right) \wedge M\left(\mathbb{Z} / 2^{r_{2}}, 1\right) \simeq M\left(\mathbb{Z} / 2^{\min \left\{r_{1}, r_{2}\right\}}, 4\right) \vee M\left(\mathbb{Z} / 2^{\min \left\{r_{1}, r_{2}\right\}}, 5\right)
$$

and so

$\pi_{5}(Y) \cong \pi_{5}\left(M\left(\mathbb{Z} / 2^{\min \left\{r_{1}, r_{2}\right\}}, 4\right)\right) \oplus \pi_{5}\left(M\left(\mathbb{Z} / 2^{\min \left\{r_{1}, r_{2}\right\}}, 5\right)\right)=\mathbb{Z} / 2 \oplus \mathbb{Z} / 2^{\min \left\{r_{1}, r_{2}\right\}}$.

Consider the case $r_{1}=r_{2}=1$. From formula (3-9) and the Freudenthal Suspension Theorem,

$$
\pi_{5}\left(\Sigma^{2} M(\mathbb{Z} / 2,1) \wedge M(\mathbb{Z} / 2,1)\right) \cong \pi_{4}(\Sigma M(\mathbb{Z} / 2,1) \wedge M(\mathbb{Z} / 2,1)) \cong \mathbb{Z} / 4 .
$$

The proof is finished.

The last case is $t=3$.

Proposition 4.7 Let $r_{1}, r_{2}, r_{3} \geq 1$ and let $r=\min \left\{r_{1}, r_{2}, r_{3}\right\}$. Then $\pi_{5}\left(\Sigma K\left(\mathbb{Z} / 2^{r_{1}}, 1\right) \wedge K\left(\mathbb{Z} / 2^{r_{2}}, 1\right) \wedge K\left(\mathbb{Z} / 2^{r_{3}}, 1\right)\right)$

$$
= \begin{cases}\mathbb{Z} / 2 \oplus \mathbb{Z} / 2^{r} & \text { if } \max \left\{r_{1}, r_{2}, r_{3}\right\}>1 \\ \mathbb{Z} / 2 \oplus \mathbb{Z} / 2 & \text { if } r_{1}=r_{2}=r_{3}=1\end{cases}
$$


Proof Let $X=\Sigma K\left(\mathbb{Z} / 2^{r_{1}}, 1\right) \wedge K\left(\mathbb{Z} / 2^{r_{2}}, 1\right) \wedge K\left(\mathbb{Z} / 2^{r_{3}}, 1\right)$. Let $f_{1}$ be the composite

$$
S^{6} \stackrel{g}{\longrightarrow} \Sigma^{3} K\left(\mathbb{Z} / 2^{r_{1}}, 1\right) \cong \Sigma K\left(\mathbb{Z} / 2^{r_{1}}, 1\right) \wedge S^{1} \wedge S^{1} \longleftrightarrow X,
$$

where $g$ is a map which induces epimorphism on $H_{6}($ ) by Lemma 4.4. Similarly, we have the maps

$$
f_{i}: S^{6} \longrightarrow \Sigma K\left(\mathbb{Z} / 2^{r_{1}}, 1\right) \wedge K\left(\mathbb{Z} / 2^{r_{2}}, 1\right) \wedge K\left(\mathbb{Z} / 2^{r_{3}}, 1\right), \quad i=2,3,
$$

by replacing $K\left(\mathbb{Z} / 2^{r_{1}}, 1\right)$ by $K\left(\mathbb{Z} / 2^{r_{i}}, 1\right)$. Let $Y=\Sigma M\left(\mathbb{Z} / 2^{r_{1}}, 1\right) \wedge M\left(\mathbb{Z} / 2^{r_{2}}, 1\right) \wedge$ $M\left(\mathbb{Z} / 2^{r_{3}}, 1\right)$ and let $j: Y \hookrightarrow X$ be the canonical inclusion. The map

$$
Y \vee S^{6} \vee S^{6} \vee S^{6} \stackrel{\left(j, f_{1}, f_{2}, f_{3}\right)}{\longrightarrow} X
$$

induces an isomorphism on $H_{k}(; \mathbb{Z} / 2)$ for $k \leq 6$ and so

$$
\left(j, f_{1}, f_{2}, f_{3}\right)_{*}: \pi_{5}\left(Y \vee S^{6} \vee S^{6} \vee S^{6}\right)=\pi_{5}(Y) \longrightarrow \pi_{5}(X)
$$

is an isomorphism.

If $\max \left\{r_{1}, r_{2}, r_{3}\right\}>1$, from decomposition (3-7),

$$
Y \simeq M\left(\mathbb{Z} / 2^{r}, 4\right) \vee M\left(\mathbb{Z} / 2^{r}, 5\right) \vee M\left(\mathbb{Z} / 2^{r}, 5\right) \vee M\left(\mathbb{Z} / 2^{r}, 6\right)
$$

and so

$$
\pi_{5}(Y)=\mathbb{Z} / 2 \oplus \mathbb{Z} / 2^{r} \oplus \mathbb{Z} / 2^{r} .
$$

If $r_{1}=r_{2}=r_{3}=1$, there is a homotopy decomposition [21, Corollary 3.7]

$$
\Sigma \mathbb{R} \mathrm{P}^{2} \wedge \mathbb{R} \mathrm{P}^{2} \wedge \mathbb{R} \mathrm{P}^{2} \simeq \Sigma \mathbb{C} \mathrm{P}^{2} \wedge \mathbb{R P}^{2} \vee \Sigma^{4} \mathbb{R} \mathrm{P}^{2} \vee \Sigma^{4} \mathbb{R} \mathrm{P}^{2}
$$

By [21, Lemma $6.34(2)]$,

$$
\pi_{5}\left(\Sigma \mathbb{C} \mathrm{P}^{2} \wedge \mathbb{R P}^{2}\right)=0
$$

and so

$$
\pi_{5}\left(\Sigma \mathbb{R} \mathrm{P}^{2} \wedge \mathbb{R} \mathrm{P}^{2} \wedge \mathbb{R} \mathrm{P}^{2}\right)=\mathbb{Z} / 2 \oplus \mathbb{Z} / 2,
$$

which finishes the proof.

Remark 4.8 For the case $X=\Sigma K(\mathbb{Z} / 2,1) \wedge K(\mathbb{Z} / 2,1) \wedge K(\mathbb{Z} / 2,1)$, the Hurewicz homomorphism

$$
\pi_{5}(X)=\mathbb{Z} / 2 \oplus \mathbb{Z} / 2 \longrightarrow H_{5}(X)=\mathbb{Z} / 2 \oplus \mathbb{Z} / 2
$$

is an isomorphism and so, in the Whitehead exact sequence,

$$
H_{6}(X) \longrightarrow \Gamma_{5}(X)=\mathbb{Z} / 2
$$


is onto. This gives an example that the morphism $H_{6}(Z) \rightarrow \Gamma_{5}(Z)$ in diagram (4-1) may not be zero, which is the only example in the case $m+t=4$. More examples will be shown up in the case $m+t=3$ in the next subsections.

\subsection{The group $\pi_{5}\left(\Sigma K\left(\mathbb{Z} / 2^{r}, 1\right)\right) \cong \pi_{5}\left(\Sigma K\left(\mathbb{Z} / 2^{r}, 1\right) \wedge K\left(\mathbb{Z} / 2^{r}, 1\right)\right)$}

Lemma 4.9 Let $X=\Sigma K\left(\mathbb{Z} / 2^{r}, 1\right) \wedge K\left(\mathbb{Z} / 2^{r}, 1\right)$ with $r \geq 1$. Then mod 2 Hurewicz homomorphism

$$
\pi_{6}(X) \stackrel{h_{6}}{\longrightarrow} H_{6}(X) \longrightarrow H_{6}(X ; \mathbb{Z} / 2)
$$

is zero.

Proof Recall that the mod 2 cohomology ring

$$
H^{*}\left(K\left(\mathbb{Z} / 2^{r}, 1\right) ; \mathbb{Z} / 2\right) \cong E\left(u_{1}\right) \otimes P\left(u_{2}\right)
$$

with the $r$-th Bockstein $\beta^{r}\left(u_{1}\right)=u_{2}$. Let $x_{i}$ (and $y_{i}$ ) denote the basis for the homology $H_{i}\left(K\left(\mathbb{Z} / 2^{r} ; \mathbb{Z} / 2\right)\right.$. The Steenrod operations and the Bockstein on lower homology are given by

$$
\begin{aligned}
\mathrm{Sq}_{*}^{2} x_{4}=x_{2} & \mathrm{Sq}_{*}^{2} y_{4}=y_{2} \\
\beta_{r}\left(x_{4}\right)=x_{3} & \beta_{r} y_{4}=y_{3} \\
\beta_{r}\left(x_{2}\right)=x_{1} & \beta_{r} y_{2}=y_{1} .
\end{aligned}
$$

The $\mathbb{Z} / 2$-vector space $s^{-1} \tilde{H}_{k}(X ; \mathbb{Z} / 2)$ with $k \leq 6$ has a basis given by the table:

$$
\left(\begin{array}{lllll}
k=6 & x_{1} y_{4} & x_{2} y_{3} & x_{3} y_{2} & x_{4} y_{1} \\
5 & x_{1} y_{3} & x_{2} y_{2} & x_{3} y_{1} & \\
4 & & & x_{1} y_{2} & x_{2} y_{1} \\
3 & & & & x_{1} y_{1}
\end{array}\right)
$$

Let $\alpha \in H_{6}(X ; \mathbb{Z} / 2)$ be a spherical class. Then

$$
s^{-1} \alpha=\epsilon_{1} x_{1} y_{4}+\epsilon_{2} x_{2} y_{3}+\epsilon_{3} x_{3} y_{2}+\epsilon_{4} x_{4} y_{1}
$$

for some $\epsilon_{i} \in \mathbb{Z} / 2$. Observe that for any spherical class,

$$
\beta_{s}(\alpha)=\mathrm{Sq}_{*}^{t}(\alpha)=0
$$

for any $s, t \geq 1$. By applying $\mathrm{Sq}_{*}^{2}$ to $\alpha$, we have

$$
\begin{aligned}
0 & =\mathrm{Sq}_{*}^{2}\left(s^{-1} \alpha\right) \\
& =\epsilon_{1} \mathrm{Sq}_{*}^{2}\left(x_{1} y_{4}\right)+\epsilon_{2} \mathrm{Sq}_{*}^{2}\left(x_{2} y_{3}\right)+\epsilon_{3} \mathrm{Sq}_{*}^{2}\left(x_{3} y_{2}\right)+\epsilon_{4} \mathrm{Sq}_{*}^{2}\left(x_{4} y_{1}\right) \\
& =\epsilon_{1} x_{1} y_{2}+0+0+\epsilon_{4} x_{2} y_{1}
\end{aligned}
$$


in $s^{-1} H_{4}(X ; \mathbb{Z} / 2)$. Thus

$$
\epsilon_{1}=\epsilon_{4}=0
$$

By applying the Bockstein $\beta_{r}$ to $\alpha$, we have

$$
\begin{aligned}
0 & =\beta_{r}\left(s^{-1} \alpha\right) \\
& =\epsilon_{1} \beta_{r}\left(x_{1} y_{4}\right)+\epsilon_{2} \beta_{r}\left(x_{2} y_{3}\right)+\epsilon_{3} \beta_{r}\left(x_{3} y_{2}\right)+\epsilon_{4} \beta_{r}\left(x_{4} y_{1}\right) \\
& =\epsilon_{1} x_{1} y_{3}+\epsilon_{2} x_{1} y_{3}+\epsilon_{3} x_{3} y_{1}+\epsilon_{4} x_{3} y_{1} \\
& =\left(\epsilon_{1}+\epsilon_{2}\right) x_{1} y_{3}+\left(\epsilon_{3}+\epsilon_{4}\right) x_{3} y_{1}
\end{aligned}
$$

and so

$$
\epsilon_{1}+\epsilon_{2}=\epsilon_{3}+\epsilon_{4}=0 .
$$

Together with Equation (4-2), we have $\epsilon_{i}=0$ for $1 \leq i \leq 4$. Thus $\alpha=0$ and hence the result holds.

Theorem 4.10 $\pi_{5}(\Sigma K(\mathbb{Z} / 2,1)) \cong \pi_{5}(\Sigma K(\mathbb{Z} / 2,1) \wedge K(\mathbb{Z} / 2,1))=\mathbb{Z} / 2 \oplus \mathbb{Z} / 2$.

Proof Let $X=\Sigma K(\mathbb{Z} / 2,1) \wedge K(\mathbb{Z} / 2,1)$. Notice that

$$
H_{6}(X)=\mathbb{Z} / 2 \oplus \mathbb{Z} / 2 \cong H_{6}(X ; \mathbb{Z} / 2) .
$$

From diagram (4-1), there is an exact sequence

$$
H_{6}(X)=\mathbb{Z} / 2 \oplus \mathbb{Z} / 2 \hookrightarrow \Gamma_{5}(X) \longrightarrow \pi_{4}(X) \longrightarrow H_{5}(X)=\mathbb{Z} / 2 \oplus \mathbb{Z} / 2 .
$$

By Corollary 3.8,

$$
\pi_{4}(X) \cong \pi_{4}(\Sigma K(\mathbb{Z} / 2,1)) \cong \mathbb{Z} / 4 .
$$

From Theorem 2.5(2), there is a short exact sequence

$$
\pi_{4}(X) \otimes \mathbb{Z} / 2 \oplus \Lambda^{2}\left(\pi_{3}(X)\right)=\mathbb{Z} / 2 \hookrightarrow \Gamma_{5}(X) \longrightarrow \operatorname{Tor}\left(\pi_{3}(X), \mathbb{Z} / 2\right)=\mathbb{Z} / 2 .
$$

Thus the group $\Gamma_{5}(X)$ is of order 4 . It follows that the monomorphism

$$
H_{6}(X)=\mathbb{Z} / 2 \oplus \mathbb{Z} / 2 \hookrightarrow \Gamma_{4}(X)
$$

is an isomorphism and hence the result holds.

Lemma 4.11 Let $r_{1}, r_{2} \geq 1$ with $\max \left\{r_{1}, r_{2}\right\}>1$. Then there is a short exact sequence

$$
\mathbb{Z} / 2 \oplus \mathbb{Z} / 2 \hookrightarrow \Gamma_{5}\left(\Sigma K\left(\mathbb{Z} / 2^{r_{1}}, 1\right) \wedge K\left(\mathbb{Z} / 2^{r_{2}}, 1\right)\right) \longrightarrow \mathbb{Z} / 2 .
$$


Proof Let $A=\mathbb{Z} / 2^{r_{1}} \oplus \mathbb{Z} / 2^{r_{2}}$. Let $X=\Sigma K\left(\mathbb{Z} / 2^{r_{1}}, 1\right) \wedge K\left(\mathbb{Z} / 2^{r_{2}}, 1\right)$. Then $X$ is a retract of $\Sigma K(A, 1) \wedge K(A, 1)$. From Theorem 3.7,

$$
\Gamma_{4}(X)=\mathbb{Z} / 2^{r_{1}} \otimes \mathbb{Z} / 2^{r_{2}} \otimes \mathbb{Z} / 2=\mathbb{Z} / 2
$$

is a summand of $\pi_{4}(X)$ and so

$$
\begin{aligned}
\pi_{4}(X) & \cong \Gamma_{4}(X) \oplus H_{4}(X) \\
& =\Gamma_{4}(X) \oplus H_{3}\left(K\left(\mathbb{Z} / 2^{r_{1}}, 1\right) \wedge K\left(\mathbb{Z} / 2^{r_{2}}, 1\right)\right) \\
& \cong \Gamma_{4}(X) \oplus \operatorname{Tor}\left(\mathbb{Z} / 2^{r_{1}}, \mathbb{Z} / 2^{r_{2}}\right) \\
& \cong \mathbb{Z} / 2 \oplus \mathbb{Z} / 2^{\min \left\{r_{1}, r_{2}\right\}}
\end{aligned}
$$

The assertion follows from Theorem 2.5(2).

There is a canonical choice of skeleton $\operatorname{sk}_{n}\left(K\left(\mathbb{Z} / 2^{r}, 1\right)\right)$ with

$$
\operatorname{sk}_{n}\left(K\left(\mathbb{Z} / 2^{r}, 1\right)=\operatorname{sk}_{n-1}\left(K\left(\mathbb{Z} / 2^{r}, 1\right) \cup e^{n} .\right.\right.
$$

This induces a choice of skeleton

$\operatorname{sk}_{n}\left(\Sigma K\left(\mathbb{Z} / 2^{r_{1}}, 1\right) \wedge K\left(\mathbb{Z} / 2^{r_{2}}, 1\right)\right)=\Sigma \bigcup_{i+j \leq n} \operatorname{sk}_{i}\left(K\left(\mathbb{Z} / 2^{r_{1}}, 1\right)\right) \wedge \mathrm{sk}_{j}\left(K\left(\mathbb{Z} / 2^{r_{2}}, 1\right)\right)$

Lemma 4.12 Let $r_{1}, r_{2} \geq 1$. Let $r=\min \left\{r_{1}, r_{2}\right\}$. Let

$$
X=\Sigma K\left(\mathbb{Z} / 2^{r_{1}}, 1\right) \wedge K\left(\mathbb{Z} / 2^{r_{2}}, 1\right) .
$$

Then

(1) $\operatorname{sk}_{4}(X) \simeq M\left(\mathbb{Z} / 2^{r}, 3\right) \vee S^{3}$;

(2) If $r_{1}=r_{2}=1$, then $\operatorname{sk}_{5}(X) \simeq S^{5} \vee S^{5} \vee \Sigma \mathbb{R} \mathrm{P}^{2} \wedge \mathbb{R} \mathrm{P}^{2}$;

(3) If $\max \left\{r_{1}, r_{2}\right\}>1$, then $\operatorname{sk}_{5}(X) \simeq S^{5} \vee S^{5} \vee M\left(\mathbb{Z} / 2^{r}, 3\right) \vee M\left(\mathbb{Z} / 2^{r}, 4\right)$;

(4) The group

$$
\begin{aligned}
\Gamma_{5}(X) \cong \pi_{5}\left(\Sigma M\left(\mathbb{Z} / 2^{r_{1}}, 1\right) \wedge M\left(\mathbb{Z} / 2^{r_{2}}, 1\right)\right) \\
= \begin{cases}\mathbb{Z} / 2 \oplus \mathbb{Z} / 2 & \text { if } r_{1}=r_{2}=1, \\
\mathbb{Z} / 4 \oplus \mathbb{Z} / 2 & \text { if } \min \left\{r_{1}, r_{2}\right\}=1 \\
& \text { and } \max \left\{r_{1}, r_{2}\right\}>1, \\
\mathbb{Z} / 2 \oplus \mathbb{Z} / 2 \oplus \mathbb{Z} / 2 & \text { if } r_{1}, r_{2}>1 .\end{cases}
\end{aligned}
$$


Proof We may assume that $r_{1} \leq r_{2}$ and so $r=r_{1}$. Let $x_{i}\left(y_{i}\right)$ be a basis for $H_{i}\left(K\left(\mathbb{Z} / 2^{r_{1}}, 1\right) ; \mathbb{Z} / 2\right)\left(H_{i}\left(K\left(\mathbb{Z} / 2^{r_{2}}, 1\right) ; \mathbb{Z} / 2\right)\right)$, which represents the $i$-dimensional cell in the space $K\left(\mathbb{Z} / 2^{r_{k}}, 1\right)$. Then

$$
s^{-1} \tilde{H}_{*}\left(\mathrm{sk}_{n+1}(X) ; \mathbb{Z} / 2\right)
$$

has a basis given by $x_{i} y_{j}$ with $i+j \leq n$ and $i, j \geq 1$. In particular, $s^{-1} \tilde{H}_{*}\left(\operatorname{sk}_{4}(X) ; \mathbb{Z} / 2\right)$ has a basis $\left\{x_{1} y_{1}, x_{1} y_{2}, x_{2} y_{1}\right\}$ with the Bockstein $\beta_{r_{1}}\left(x_{2} y_{1}\right)=x_{1} y_{1}$. There is (unique up to homotopy) 2-local 3-cell complex with this homological structure which is given by $S^{4} \vee M\left(\mathbb{Z} / 2^{r}, 3\right)$. Thus $\operatorname{sk}_{4}(X) \simeq S^{4} \vee M\left(\mathbb{Z} / 2^{r}, 3\right)$, which is assertion (1).

For (2) and (3), observe that $s^{-1} \tilde{H}_{*}\left(\operatorname{sk}_{5}(X) ; \mathbb{Z} / 2\right)$ has a basis

$$
\left\{x_{1} y_{1}, x_{1} y_{2}, x_{2} y_{1}, x_{1} y_{3}, x_{2} y_{2}, x_{3} y_{3}\right\} \text {. }
$$

Let

$$
j: \Sigma M\left(\mathbb{Z} / 2^{r_{1}}, 1\right) \wedge M\left(\mathbb{Z} / 2^{r_{2}}, 1\right) \hookrightarrow \operatorname{sk}_{5}(X)
$$

be the canonical inclusion. For $i=1,2$, the composite

$S^{5} \stackrel{g}{\longrightarrow} \Sigma^{2} K\left(\mathbb{Z} / 2^{r_{i}}, 1\right) \cong \Sigma K\left(\mathbb{Z} / 2^{r_{i}}, 1\right) \wedge S^{1} \hookrightarrow \Sigma K\left(\mathbb{Z} / 2^{r_{1}}, 1\right) \wedge K\left(\mathbb{Z} / 2^{r_{2}}, 1\right)$, in which $g$ is map that inducing isomorphism on $H_{5}(; \mathbb{Z} / 2)$ as in Lemma 4.4, induces a map

$$
f_{i}: S^{5} \longrightarrow \operatorname{sk}_{5}(X) .
$$

By inspecting homology, the map

$$
\left(f_{1}, f_{2}, j\right): S^{5} \vee S^{5} \vee \Sigma M\left(\mathbb{Z} / 2^{r_{1}}, 1\right) \wedge M\left(\mathbb{Z} / 2^{r_{2}}, 1\right) \longrightarrow \operatorname{sk}_{5}(X)
$$

induces an isomorphism on mod 2 homology and so it is a homotopy equivalent localized at 2. If $\max \left\{r_{1}, r_{2}\right\}>1$, then from decomposition (3-7),

$$
\Sigma M\left(\mathbb{Z} / 2^{r_{1}}, 1\right) \wedge M\left(\mathbb{Z} / 2^{r_{2}}, 1\right) \simeq M\left(\mathbb{Z} / 2^{r}, 3\right) \vee M\left(\mathbb{Z} / 2^{r}, 4\right)
$$

and so $\operatorname{sk}_{5}(X) \simeq S^{5} \vee S^{5} \vee M\left(\mathbb{Z} / 2^{r}, 3\right) \vee M\left(\mathbb{Z} / 2^{r}, 4\right)$ in this case. Thus assertions (2) and (3) follow.

(4) splits into two cases.

Case I $\max \left\{r_{1}, r_{2}\right\}>1$. By the definition of the Whitehead's functor $\Gamma$,

$$
\begin{aligned}
\Gamma_{5}(X) & =\operatorname{Im}\left(\pi_{5}\left(\operatorname{sk}_{4}(X)\right) \rightarrow \pi_{5}\left(\operatorname{sk}_{5}(X)\right)\right. \\
& =\operatorname{Im}\left(\pi_{5}\left(S^{4} \vee M\left(\mathbb{Z} / 2^{r}, 3\right)\right) \rightarrow \pi_{5}\left(S^{5} \vee S^{5} \vee M\left(\mathbb{Z} / 2^{r}, 3\right) \vee M\left(\mathbb{Z} / 2^{r}, 4\right)\right)\right) \\
& =\pi_{5}\left(M\left(\mathbb{Z} / 2^{r}, 3\right) \vee M\left(\mathbb{Z} / 2^{r}, 4\right)\right)
\end{aligned}
$$


because

$$
\begin{aligned}
\left.\left.M\left(\mathbb{Z} / 2^{r}, 3\right) \vee M\left(\mathbb{Z} / 2^{r}, 4\right)\right)\right) & \simeq \Sigma M\left(\mathbb{Z} / 2^{r_{1}}, 1\right) \wedge M\left(\mathbb{Z} / 2^{r_{2}}, 1\right) \\
& =\left(S^{4} \vee M\left(\mathbb{Z} / 2^{r}, 3\right)\right) \cup e^{5} .
\end{aligned}
$$

Now it suffices to compute

$$
\pi_{5}\left(M\left(\mathbb{Z} / 2^{r}, 3\right) \vee M\left(\mathbb{Z} / 2^{r}, 4\right)\right)=\pi_{5}\left(M\left(\mathbb{Z} / 2^{r}, 3\right)\right) \oplus \pi_{5}\left(M\left(\mathbb{Z} / 2^{r}, 4\right)\right) .
$$

It is straight forward to see that $\pi_{5}\left(M\left(\mathbb{Z} / 2^{r}, 4\right)\right)=\mathbb{Z} / 2$ represented by the composite

$$
S^{5} \stackrel{\eta}{\longrightarrow} S^{4} \longrightarrow M\left(\mathbb{Z} / 2^{r}, 4\right) .
$$

If $r=\min \left\{r_{1}, r_{2}\right\}=1$, then $\pi_{5}\left(M\left(\mathbb{Z} / 2^{r}, 3\right)=\mathbb{Z} / 4\right.$ according to [21, Proposition 5.1].

If $r=\min \left\{r_{1}, r_{2}\right\}>1$, we compute $\pi_{5}\left(M \mathbb{Z} / 2^{r}, 3\right)$. Observe that this is in the stable range and so

$$
\pi_{5}\left(M\left(\mathbb{Z} / 2^{r}, 3\right)\right) \cong \pi_{5}^{s}\left(M\left(\mathbb{Z} / 2^{r}, 3\right)\right) .
$$

Now we are working in the stable homotopy category. Since $\eta: S^{5} \rightarrow S^{4}$ is of order 2, there is a map

$$
\tilde{\eta}: M(\mathbb{Z} / 2,5) \longrightarrow S^{4}
$$

such that $\left.\tilde{\eta}\right|_{S^{5}} \simeq \eta$. Since the identity map of $M(\mathbb{Z} / 2,5)$ is of order 4 (see for instance Toda [16, Theorem 4.4]), there is a commutative diagram

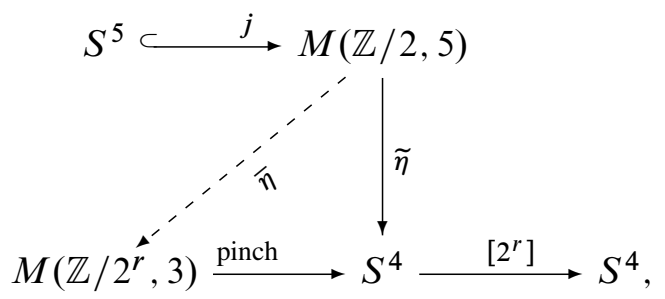

where the bottom row is the cofibre sequence. The composite $\bar{\eta}: S^{5} \rightarrow M\left(\mathbb{Z} / 2^{r}, 3\right)$ represents an element in $\pi_{5}^{s}\left(M\left(\mathbb{Z} / 2^{r}, 3\right)\right)$ that maps down to $\pi_{5}^{s}\left(S^{4}\right)=\mathbb{Z} / 2(\eta)$. Since the map $j: S^{5} \rightarrow M(\mathbb{Z} / 2,5)$ is of order 2 , the composite $\bar{\eta} \circ j$ is of order 2 . It follows that

$$
\pi_{5}\left(M\left(\mathbb{Z} / 2^{r}, 3\right)\right) \cong \pi_{5}^{s}\left(M\left(\mathbb{Z} / 2^{r}, 3\right)\right) \cong \pi_{5}^{s}\left(S^{4}\right) \oplus \pi_{5}^{s}\left(S^{3}\right) \cong \mathbb{Z} / 2 \oplus \mathbb{Z} / 2 .
$$

Case II $r_{1}=r_{2}=1$. In this case, similar to the above arguments,

$$
\Gamma_{5}(X)=\Gamma_{5}\left(\Sigma \mathbb{R} \mathrm{P}^{2} \wedge \mathbb{R} \mathrm{P}^{2}\right)=\pi_{5}\left(\Sigma \mathbb{R} \mathrm{P}^{2} \wedge \mathbb{R} \mathrm{P}^{2}\right) .
$$


We compute this homotopy group. Note that

$$
\Sigma \mathbb{R} \mathrm{P}^{2} \wedge \mathbb{R P}^{2}=\operatorname{sk}_{4}(X) \cup e^{5}=\left(S^{4} \vee M(\mathbb{Z} / 2,3) \cup e^{5} .\right.
$$

There is a cofibre sequence

$$
S^{4} \stackrel{f}{\longrightarrow} S^{4} \vee M(\mathbb{Z} / 2,3) \stackrel{g}{\longrightarrow} \Sigma \mathbb{R}^{2} \wedge \mathbb{R}^{2},
$$

where the composite

$$
S^{4} \stackrel{f}{\longrightarrow} S^{4} \vee M(\mathbb{Z} / 2,3) \stackrel{\text { proj }}{\longrightarrow} S^{4}
$$

is of degree 2 because

$$
\mathrm{Sq}_{*}^{1}: H_{5}\left(\Sigma \mathbb{R} \mathrm{P}^{2} \wedge \mathbb{R} \mathrm{P}^{2} ; \mathbb{Z} / 2\right)=\mathbb{Z} / 2 \longrightarrow H_{4}\left(\Sigma \mathbb{R} \mathrm{P}^{2} \wedge \mathbb{R} \mathrm{P}^{2} ; \mathbb{Z} / 2\right)=\mathbb{Z} / 2 \oplus \mathbb{Z} / 2
$$
is not zero, and the composite

$$
S^{4} \stackrel{f}{\longrightarrow} S^{4} \vee M(\mathbb{Z} / 2,3) \stackrel{\text { proj }}{\longrightarrow} M(\mathbb{Z} / 2,3)
$$

is homotopic to the composite

$$
S^{4} \stackrel{\eta}{\longrightarrow} S^{3} \stackrel{j}{\longrightarrow} M(\mathbb{Z} / 2,3)
$$

because $\pi_{4}(M(\mathbb{Z} / 2,3))=\mathbb{Z} / 2$ and

$$
\mathrm{Sq}_{*}^{2}: H_{5}\left(\Sigma \mathbb{R} \mathrm{P}^{2} \wedge \mathbb{R} \mathrm{P}^{2} ; \mathbb{Z} / 2\right)=\mathbb{Z} / 2 \longrightarrow H_{3}\left(\Sigma \mathbb{R} \mathrm{P}^{2} \wedge \mathbb{R} \mathrm{P}^{2} ; \mathbb{Z} / 2\right)=\mathbb{Z} / 2
$$

is an isomorphism. Since

$$
\begin{gathered}
\Gamma_{5}\left(\Sigma \mathbb{R} \mathrm{P}^{2} \wedge \mathbb{R} \mathrm{P}^{2}\right)=\pi_{5}\left(\Sigma \mathbb{R} \mathrm{P}^{2} \wedge \mathbb{R P}^{2}\right), \\
g_{*}: \pi_{5}\left(S^{4} \vee M(\mathbb{Z} / 2,3)\right) \longrightarrow \pi_{5}\left(\Sigma \mathbb{R} \mathrm{P}^{2} \wedge \mathbb{R} \mathrm{P}^{2}\right)
\end{gathered}
$$

is an epimorphism. By applying the Hilton-Milnor Theorem,

$$
\begin{aligned}
\pi_{5}\left(S^{4} \vee M(\mathbb{Z} / 2,3)\right) & \cong \pi_{4}\left(\Omega\left(S^{4} \vee M(\mathbb{Z} / 2,3)\right)\right) \\
& \cong \pi_{4}\left(\Omega S^{4} \times \Omega(M(\mathbb{Z} / 2,3)) \times \Omega \Sigma\left(\Omega S^{4} \wedge \Omega M(\mathbb{Z} / 2,3)\right)\right) \\
& \cong \pi_{4}\left(\Omega S^{4}\right) \oplus \pi_{4}(\Omega(M(\mathbb{Z} / 2,3))) \\
& \cong \pi_{5}\left(S^{4}\right) \oplus \pi_{5}(M(\mathbb{Z} / 2,3)) \\
& \cong \mathbb{Z} / 2 \oplus \pi_{5}(M(\mathbb{Z} / 2,3)) .
\end{aligned}
$$

From [21, Proposition 5.1], $\pi_{5}(M(\mathbb{Z} / 2,3))=\mathbb{Z} / 4$ generated by the homotopy class of any map $\phi: S^{5} \rightarrow M(\mathbb{Z} / 2,3)$ such that the composite

$$
S^{5} \rightarrow M(\mathbb{Z} / 2,3) \rightarrow S^{4}
$$


is homotopic to $\eta$, the generator for $\pi_{5}\left(S^{4}\right)=\mathbb{Z} / 2$, and, for any such a choice of map $\phi$, the element $2[\phi]$ is given by the homotopy class of the composite

$$
S^{5} \stackrel{\eta}{\longrightarrow} S^{4} \stackrel{\eta}{\longrightarrow} S^{3} \stackrel{j}{\longrightarrow} M(\mathbb{Z} / 2,3) .
$$

From the fact that $g \circ f \simeq *$, the composite

$$
\pi_{5}\left(S^{4}\right) \stackrel{f_{*}}{\longrightarrow} \pi_{5}\left(\pi_{5}\left(S^{4} \vee M(\mathbb{Z} / 2,3)\right)\right) \stackrel{g_{*}}{\longrightarrow} \pi_{5}\left(\Sigma \mathbb{R} \mathrm{P}^{2} \wedge \mathbb{R P}^{2}\right)
$$

is zero. Observe that

$$
f_{*}(\eta)=2 \eta+[j \circ \eta \circ \eta]=2[\phi] .
$$

Thus $g_{*}(2[\phi])=0$ and so $\pi_{5}\left(\Sigma \mathbb{R} \mathrm{P}^{2} \wedge \mathbb{R P}^{2}\right)$ is a quotient group $\mathbb{Z} / 2 \oplus \mathbb{Z} / 2$. On the other hand, from Theorem 2.5(2), there is short exact sequence

$$
\mathbb{Z} / 2 \hookrightarrow \Gamma_{5}\left(\Sigma \mathbb{R} \mathrm{P}^{2} \wedge \mathbb{R} \mathrm{P}^{2}\right)=\pi_{5}\left(\Sigma \mathbb{R} \mathrm{P}^{2} \wedge \mathbb{R} \mathrm{P}^{2}\right) \longrightarrow \mathbb{Z} / 2 .
$$

It follows that $\pi_{5}\left(\Sigma \mathbb{R} \mathrm{P}^{2} \wedge \mathbb{R} \mathrm{P}^{2}\right)=\mathbb{Z} / 2 \oplus \mathbb{Z} / 2$. The proof is finished.

Let $\operatorname{Len}^{3}\left(2^{r}\right)=\operatorname{sk}_{3}\left(K\left(\mathbb{Z} / 2^{r}, 1\right)\right)$ be the 3 -dimensional lens space.

Lemma 4.13 Let $r_{1}, r_{2} \geq 1$. Let

$$
\begin{aligned}
X_{1} & =\Sigma M\left(\mathbb{Z} / 2^{r_{1}}, 1\right) \wedge M\left(\mathbb{Z} / 2^{r_{2}}, 1\right), \\
X_{2} & =\Sigma \operatorname{Len}^{3}\left(2^{r_{1}}\right) \wedge \operatorname{Len}^{3}\left(2^{r_{2}}\right), \\
X & =\Sigma K\left(\mathbb{Z} / 2^{r_{1}}, 1\right) \wedge K\left(\mathbb{Z} / 2^{r_{2}}, 1\right) .
\end{aligned}
$$

Then there is a commutative diagram

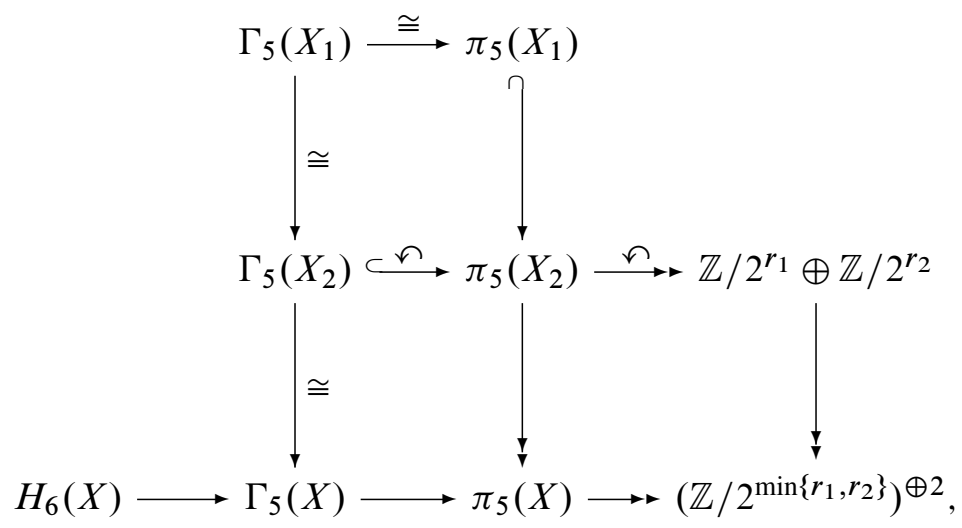

where the rows are exact and the middle a splitting short exact sequence. 
Proof As in the proof in Lemma 4.12, $s^{-1} \tilde{H}_{k}(X)$ for $k \leq 6$ has a basis

$$
\left\{x_{i} y_{j} \mid i+j \leq 6, i, j \geq 1\right\} \text {. }
$$

Thus

$$
\operatorname{sk}_{4}(X) \subseteq X_{1} \subseteq \operatorname{sk}_{5}(X) \subseteq X_{2} \subseteq \operatorname{sk}_{7}(X)
$$

and so the commutative diagram follows, where

$$
\Gamma_{5}\left(X_{1}\right) \cong \Gamma_{5}\left(X_{2}\right) \cong \Gamma_{5}(X)
$$

are given by Lemma 4.12. Since $\operatorname{sk}_{5}(X) \subseteq X_{2}, \pi_{5}\left(X_{2}\right) \rightarrow \pi_{5}(X)$ is onto.

Now we show that the middle row in the diagram splits off. By taking the suspension, there is a commutative diagram of short exact sequences

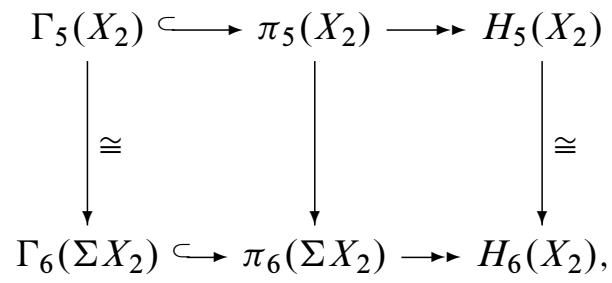

where the left column is an isomorphism because

$$
\Gamma_{5}\left(X_{2}\right) \cong \pi_{5}\left(X_{1}\right) \cong \pi_{6}\left(\Sigma X_{1}\right) \cong \Gamma_{6}\left(\Sigma X_{2}\right) .
$$

Thus by the 5 -Lemma,

$$
\pi_{5}\left(X_{2}\right) \cong \pi_{6}\left(\Sigma X_{2}\right)
$$

From Lemma 4.4, there is a map

$$
g: S^{5} \longrightarrow \Sigma^{2} K\left(\mathbb{Z} / 2^{r}, 1\right)
$$

inducing an isomorphism on $H_{5}(; \mathbb{Z} / 2)$. It follows that

$$
\Sigma^{2} \operatorname{Len}^{3}\left(2^{r}\right)=\Sigma^{2} \operatorname{sk}_{3}\left(K\left(\mathbb{Z} / 2^{r}, 1\right)\right) \simeq S^{5} \vee \Sigma^{2} M\left(\mathbb{Z} / 2^{r}, 1\right)
$$

and so

$$
\begin{aligned}
\Sigma X_{2} & =\Sigma^{2} \operatorname{Len}^{3}\left(2^{r_{1}}\right) \wedge \operatorname{Len}^{3}\left(2^{r_{2}}\right) \\
& \simeq\left(S^{5} \vee \Sigma^{2} M\left(\mathbb{Z} / 2^{r_{1}}, 1\right)\right) \wedge \operatorname{Len}^{3}\left(2^{r_{2}}\right) \\
& \simeq \Sigma^{5} \operatorname{Len}^{3}\left(2^{r_{2}}\right) \vee \Sigma^{2} \operatorname{Len}^{3}\left(2^{r_{2}}\right) \wedge M\left(\mathbb{Z} / 2^{r_{1}}, 1\right) \\
& \simeq S^{8} \vee M\left(\mathbb{Z} / 2^{r_{2}}, 6\right) \vee M\left(\mathbb{Z} / 2^{r_{1}}, 6\right) \vee M\left(\mathbb{Z} / 2^{r_{2}}, 3\right) \wedge M\left(\mathbb{Z} / 2^{r_{1}}, 1\right) .
\end{aligned}
$$

Thus

$$
\pi_{6}\left(\Sigma X_{2}\right) \cong \mathbb{Z} / 2^{r_{1}} \oplus \mathbb{Z} / 2^{r_{2}} \oplus \Gamma_{6}\left(\Sigma X_{2}\right)
$$


and hence the result holds.

Theorem 4.14 Let $r>1$. Then

$$
\pi_{5}\left(\Sigma K\left(\mathbb{Z} / 2^{r}, 1\right)\right) \cong \pi_{5}\left(\Sigma K\left(\mathbb{Z} / 2^{r}, 1\right) \wedge K\left(\mathbb{Z} / 2^{r}, 1\right)\right) \cong \mathbb{Z} / 2 \oplus \mathbb{Z} / 2^{r} \oplus \mathbb{Z} / 2^{r}
$$

Proof Let $X=\Sigma K\left(\mathbb{Z} / 2^{r}, 1\right) \wedge K\left(\mathbb{Z} / 2^{r}, 1\right)$. By Lemma 4.13,

$$
\pi_{5}(X) \cong \mathbb{Z} / 2^{r} \oplus \mathbb{Z} / 2^{r} \oplus \operatorname{Im}\left(\Gamma_{5}(X) \rightarrow \pi_{5}(X)\right) .
$$

From Lemma 4.12,

$$
\Gamma_{5}(X)=\mathbb{Z} / 2^{\oplus 3}
$$

By Lemma 4.4, the composite

$$
\pi_{6}(X) \rightarrow H_{6}(X)=\mathbb{Z} / 2^{r} \oplus \mathbb{Z} / 2^{r} \rightarrow H_{6}(X ; \mathbb{Z} / 2)
$$

is zero. Thus

$$
H_{6}(X)=\mathbb{Z} / 2^{r} \oplus \mathbb{Z} / 2^{r} \longrightarrow \Gamma_{5}(X)=\mathbb{Z} / 2 \oplus \mathbb{Z} / 2 \oplus \mathbb{Z} / 2
$$

detects two copies of $\mathbb{Z} / 2$-summands in $\Gamma_{5}(X)$. The proof is finished.

\subsection{The group $\pi_{5}\left(\Sigma K\left(\mathbb{Z} / 2^{r_{1}}, 1\right) \wedge K\left(\mathbb{Z} / 2^{r_{2}}, 1\right)\right)$ with $r_{1}<r_{2}$}

Our computation is given by analyzing the cell structure. Let $x_{i}$ be a basis for $\tilde{H}_{i}\left(K\left(\mathbb{Z} / 2^{r_{1}}, \mathbb{Z} / 2\right)\right)$ and let $y_{i}$ be a basis for $\tilde{H}_{i}\left(K\left(\mathbb{Z} / 2^{r_{2}} ; \mathbb{Z} / 2\right)\right)$. Then

$$
s^{-1} \tilde{H}_{k}\left(\Sigma K\left(\mathbb{Z} / 2^{r_{1}}, 1\right) \wedge K\left(\mathbb{Z} / 2^{r_{2}}, 1\right), \quad k \leq 6,\right.
$$


has a basis $\left\{x_{i} y_{j} \mid i+j \leq 6\right\}$. From the assumption that $r_{1}<r_{2}$, the Steenrod operation and Bockstein are indicated by the following diagram

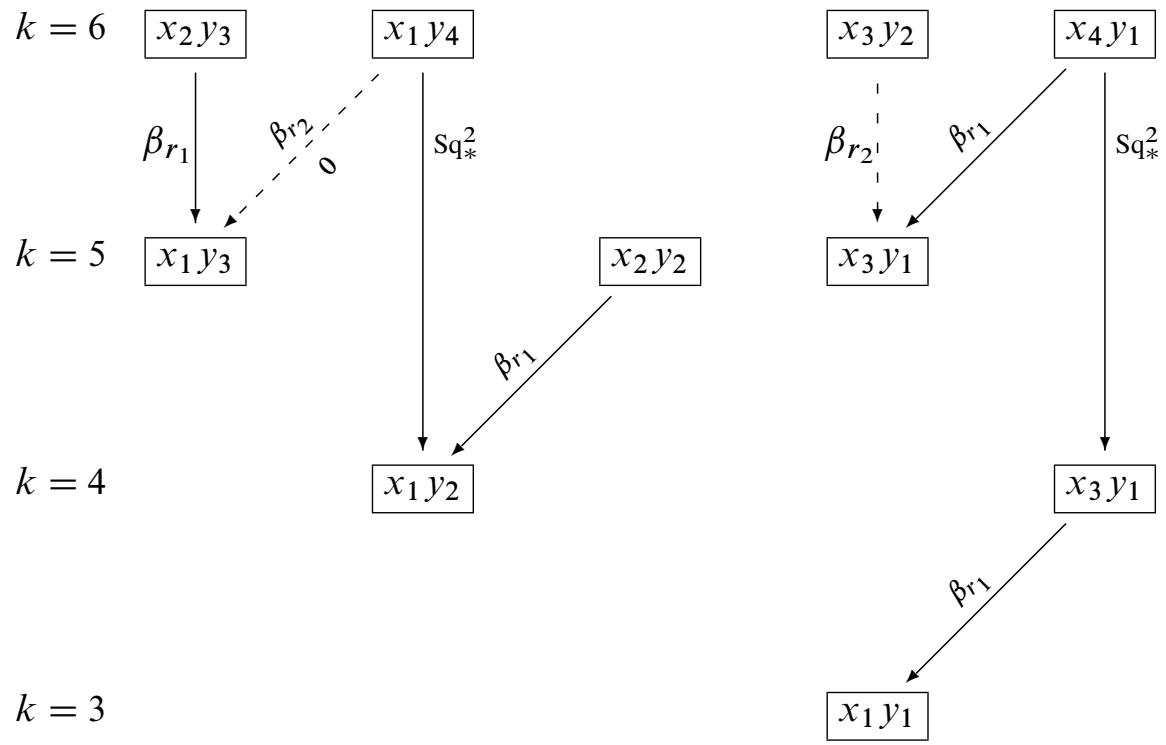

$(4-8)$

$k=3$

where the dashed arrows mean that the next Bockstein $\beta_{r_{2}}$, which comes from $H_{*}\left(K\left(\mathbb{Z} / 2^{r_{2}}, 1\right)\right)$, does not actually happen in the Bockstein spectral sequence up to this range.

Lemma 4.15 Let $r_{2}>r_{1} \geq 1$ and let $X=\Sigma K\left(\mathbb{Z} / 2^{r_{1}}, 1\right) \wedge K\left(\mathbb{Z} / 2^{r_{2}}, 1\right)$. Then the suspension

$$
E: \pi_{5}(X) \longrightarrow \pi_{6}(\Sigma X)
$$

is an isomorphism.

Proof From formula (4-5) together with the fact that $\pi_{n-1}\left(\mathrm{sk}_{n}(Y)\right) \cong \pi_{n-1}(Y)$,

$$
\pi_{5}\left(\operatorname{sk}_{6}\left(\Sigma \operatorname{Len}^{3}\left(2^{r_{1}}\right) \wedge \operatorname{Len}^{3}\left(2^{r_{2}}\right)\right)\right) \longrightarrow \pi_{6}\left(\operatorname{sk}_{7}\left(\Sigma \operatorname{Len}^{3}\left(2^{r_{1}}\right) \wedge \operatorname{Len}^{3}\left(2^{r_{2}}\right)\right)\right) .
$$

Notice that

$$
\operatorname{sk}_{6}(X)=\operatorname{sk}_{6}\left(\Sigma \operatorname{Len}^{3}\left(2^{r_{1}}\right) \wedge \operatorname{Len}^{3}\left(2^{r_{2}}\right)\right) \cup e^{6} \cup e^{6}
$$


indicated by the elements $x_{1} y_{4}$ and $x_{4} y_{1}$ in diagram (4-8). Then there is a commutative diagram of right exact sequences

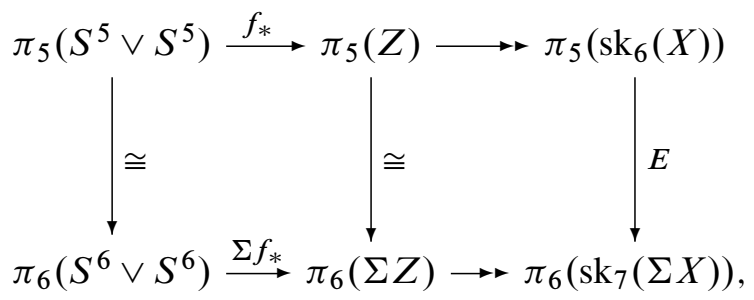

where $Z=\operatorname{sk}_{6}\left(\Sigma \operatorname{Len}^{3}\left(2^{r_{1}}\right) \wedge \operatorname{Len}^{3}\left(2^{r_{2}}\right)\right)$ and $f: S^{5} \vee S^{5} \rightarrow Y$ is the attaching map for $\operatorname{sk}_{6}(X)$. The assertion follows by the 5 -lemma.

Theorem 4.16 Let $r_{2}>r_{1} \geq 1$. Then

$\pi_{5}\left(\Sigma K\left(\mathbb{Z} / 2^{r_{1}}, 1\right) \wedge K\left(\mathbb{Z} / 2^{r_{2}}, 1\right)\right)= \begin{cases}\mathbb{Z} / 2 \oplus \mathbb{Z} / 4 & \text { if } r_{1}=1, r_{2}=2, \\ \mathbb{Z} / 2 \oplus \mathbb{Z} / 8 & \text { if } r_{1}=1, r_{2} \geq 3, \\ \mathbb{Z} / 2 \oplus \mathbb{Z} / 2^{r_{1}} \oplus \mathbb{Z} / 2^{r_{1}+1} & \text { if } r_{2}>r_{1}>1\end{cases}$

Proof From Lemma 4.15, it suffices to compute $\pi_{6}\left(\Sigma^{2} K\left(\mathbb{Z} / 2^{r_{1}}, 1\right) \wedge K\left(\mathbb{Z} / 2^{r_{2}}, 1\right)\right)$. Let $X=\operatorname{sk}_{7}\left(\Sigma^{2} K\left(\mathbb{Z} / 2^{r_{1}}, 1\right) \wedge K\left(\mathbb{Z} / 2^{r_{2}}, 1\right)\right)$. From splitting formula (4-7), $\operatorname{sk}_{7}\left(\Sigma^{2} \operatorname{Len}^{3}\left(2^{r_{1}}\right) \wedge \operatorname{Len}^{3}\left(2^{r_{2}}\right)\right)$

$$
\simeq M\left(\mathbb{Z} / 2^{r_{2}}, 6\right) \vee M\left(\mathbb{Z} / 2^{r_{1}}, 6\right) \vee M\left(\mathbb{Z} / 2^{r_{1}}, 4\right) \vee M\left(\mathbb{Z} / 2^{r_{1}}, 5\right) .
$$

Let $Y=\operatorname{sk}_{7}\left(\Sigma^{2} \operatorname{Len}^{3}\left(2^{r_{1}}\right) \wedge \operatorname{Len}^{3}\left(2^{r_{2}}\right)\right)$. Then $s^{-2} \tilde{H}_{*}(Y ; \mathbb{Z} / 2)$ has a basis listed in diagram (4-8) excluding the elements $x_{1} y_{4}$ and $x_{4} y_{1}$. Let $P^{n}\left(2^{r}\right)=M\left(\mathbb{Z} / 2^{r}, n-1\right)$. The mod homology $\widetilde{H}_{*}\left(P^{n}\left(2^{r}\right) ; \mathbb{Z} / 2\right)$ has a basis $u_{n-1}^{r}$ and $v_{n}^{r}$ whose degrees are $\left|u_{n-1}^{r}\right|=n-1, v_{n}^{r} \mid=n$ and the Bockstein $\beta_{r}\left(v_{n}^{r}\right)=u_{n-1}^{r}$. Since $X=Y \cup e^{7} \cup e^{7}$, there is a cofibre sequence

$$
S_{1}^{6} \vee S_{2}^{6} \stackrel{f}{\longrightarrow} P^{7}\left(2^{r_{1}}\right) \vee P^{7}\left(2^{r_{2}}\right) \vee P^{5}\left(2^{r_{1}}\right) \vee P^{6}\left(2^{r_{1}}\right) \stackrel{g}{\longrightarrow} X \stackrel{q}{\longrightarrow} S_{1}^{7} \vee S_{2}^{7},
$$

where $g_{*}\left(u_{6}^{r_{1}}, v_{7}^{r_{2}} ; u_{6}^{r_{2}}, v_{7}^{r_{2}} ; u_{4}^{r_{1}}, v_{5}^{r_{1}} ; u_{5}^{r_{1}}, v_{6}^{r_{1}}\right)$

$$
=s^{2}\left(x_{1} y_{3}, x_{2} y_{3} ; x_{3} y_{1}, x_{3} y_{2} ; x_{1} y_{1}, x_{2} y_{1} ; x_{1} y_{2}, x_{2} y_{2}\right)
$$

for catching the corresponding elements in $\widetilde{H}_{*}(X ; \mathbb{Z} / 2)$. Here the map $f$ is the attaching map with $f\left|S_{1}^{6}, f\right|_{S_{2}^{6}}$ corresponding to the homological classes $s^{2}\left(x_{4} y_{1}\right)$ and $s^{2}\left(x_{1} y_{4}\right)$, respectively. Namely, the induced boundary map $q: X \rightarrow S_{1}^{7} \vee S_{2}^{7}$ has the homological property that $q_{*}: H_{7}(X ; \mathbb{Z} / 2) \rightarrow H_{7}\left(S_{1}^{7} \vee S_{2}^{7}\right)$ is given by

$$
q_{*}\left(x_{2} y_{3}\right)=q_{*}\left(x_{3} y_{2}\right)=0, q_{*}\left(s^{2}\left(x_{4} y_{1}\right)\right)=\iota_{1}, \quad q_{*}\left(s^{2}\left(x_{1} y_{4}\right)\right)=\iota_{2},
$$


where $\iota_{j}$ is the basis for $H_{7}\left(S_{j}^{7} ; \mathbb{Z} / 2\right)$. For $j=1,2$, let $X_{j}$ be the homotopy cofibre of $f_{j}$. Then there is a commutative diagram

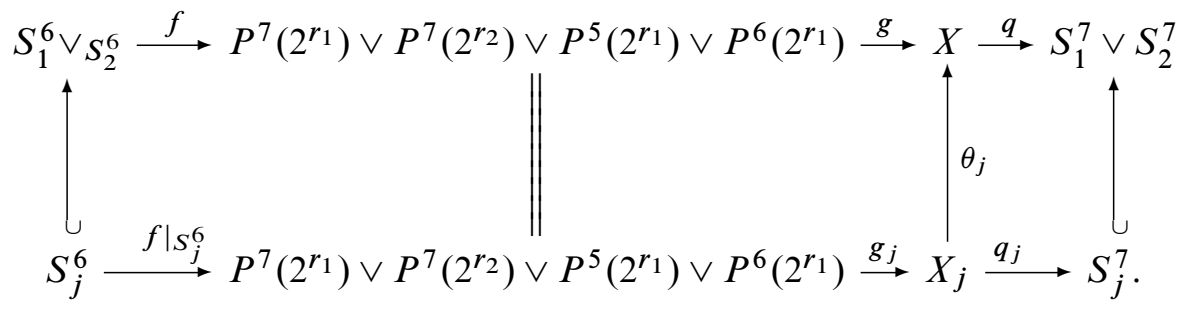

Statement $1 \theta_{j *}: \tilde{H}_{*}\left(X_{j} ; \mathbb{Z} / 2\right) \rightarrow \widetilde{H}_{*}(X ; \mathbb{Z} / 2)$ is a monomorphism. Moreover,

$$
\operatorname{Im}\left(\theta_{1 *}: H_{7}\left(X_{1} ; \mathbb{Z} / 2\right) \rightarrow H_{7}(X ; \mathbb{Z} / 2)\right)
$$

has the basis given by $\left\{s^{2}\left(x_{2} y_{3}\right), s^{2}\left(x_{3} y_{2}\right), s^{2}\left(x_{4} y_{1}\right)\right\}$ and

$$
\operatorname{Im}\left(\theta_{2 *}: H_{7}\left(X_{2} ; \mathbb{Z} / 2\right) \rightarrow H_{7}(X ; \mathbb{Z} / 2)\right)
$$

has the basis given by $\left\{s^{2}\left(x_{2} y_{3}\right), s^{2}\left(x_{3} y_{2}\right), s^{2}\left(x_{1} y_{4}\right)\right\}$. Thus a basis for $\tilde{H}_{*}\left(X_{j} ; \mathbb{Z} / 2\right)$ can be listed in diagram (4-8) by removing one element.

The statement follows immediately by applying mod 2 homology to diagram (4-9), where the only simple computation is given by checking the image of $\theta_{j *}$.

Statement 2 The composite

$$
\phi_{j}: S_{j}^{6} \stackrel{f \mid S_{j}^{6}}{\longrightarrow} P^{7}\left(2^{r_{1}}\right) \vee P^{7}\left(2^{r_{2}}\right) \vee P^{5}\left(2^{r_{1}}\right) \vee P^{6}\left(2^{r_{1}}\right) \stackrel{\text { proj }}{\longrightarrow} P^{7}\left(2^{r_{1}}\right)
$$

is null homotopic for $j=1,2$.

Consider the commutative diagram of cofibre sequences

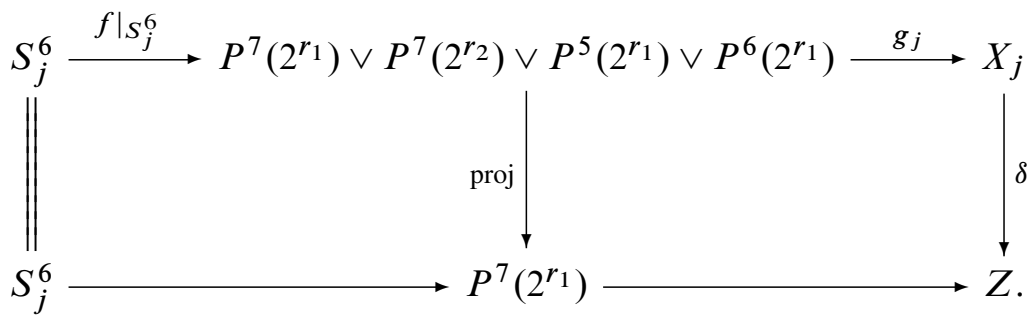

Then $\operatorname{dim} \widetilde{H}_{*}(Z ; \mathbb{Z} / 2)=3$ and $\delta_{*}: H_{*}\left(X_{j} ; \mathbb{Z} / 2\right) \rightarrow H_{*}(Z ; \mathbb{Z} / 2)$ is onto. From diagram (4-8), the Bockstein

$$
\beta_{t}: H_{7}(Z ; \mathbb{Z} / 2) \rightarrow H_{6}(Z ; \mathbb{Z} / 2)
$$


is 0 for $t<r_{1}$ with the first nontrivial Bockstein given by $\beta_{r_{1}}$ coming from $\beta_{r_{1}}\left(x_{2} y_{3}\right)=$ $x_{1} y_{3}$ in diagram (4-8). Note that $\pi_{6}\left(P^{7}\left(2^{r_{1}}\right)\right)=\mathbb{Z} / 2^{r_{1}}$ generated by the inclusion $\bar{l}: S^{6} \hookrightarrow P^{7}\left(2^{r_{1}}\right)$. Then the homotopy class

$$
\left[\phi_{j}\right]=k \bar{\imath}
$$

for some $k \in \mathbb{Z}$. If $k \equiv 1 \bmod 2$, then $\operatorname{dim} \widetilde{H}_{*}(Z ; \mathbb{Z} / 2)=1$ which contradicts to that $\operatorname{dim} \widetilde{H}_{*}(Z ; \mathbb{Z} / 2)=3$. Thus $k$ must be divisible by 2 . Let $k=2^{t} k^{\prime}$ with $k^{\prime} \equiv 1 \bmod 2$ for some $t \geq 1$. If $t<r_{1}$, then there is a nontrivial Bockstein $\beta_{t}$ on $\widetilde{H}_{*}(Z ; \mathbb{Z} / 2)$ which is impossible from the above. Hence $t \geq r_{1}$ and so $\left[\phi_{j}\right]=0$ in $\pi_{6}\left(P^{7}\left(2^{r_{1}}\right)\right)=\mathbb{Z} / 2^{r_{1}}$. Statement 2 follows.

Statement 3 The composite

$$
\psi: S_{1}^{6} \stackrel{\left.f\right|_{S_{1}^{6}}}{\longrightarrow} P^{7}\left(2^{r_{1}}\right) \vee P^{7}\left(2^{r_{2}}\right) \vee P^{5}\left(2^{r_{1}}\right) \vee P^{6}\left(2^{r_{1}}\right) \stackrel{\text { proj }}{\longrightarrow} P^{6}\left(2^{r_{1}}\right)
$$

is null homotopic.

Consider the commutative diagram of cofibre sequences

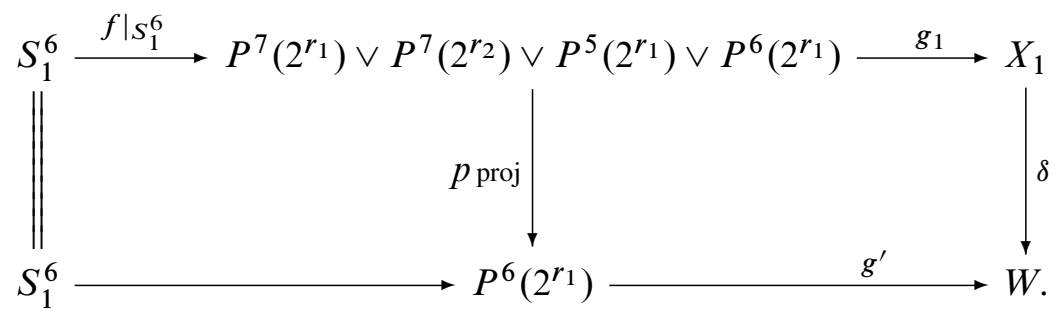

Then $\operatorname{dim} \widetilde{H}_{*}(W ; \mathbb{Z} / 2)=3$ and $\left.\delta_{*}: \widetilde{H}_{(} X_{1} ; \mathbb{Z} / 2\right) \rightarrow \widetilde{H}_{*}(W ; \mathbb{Z} / 2)$ is onto. Moreover, $H_{7}(W ; \mathbb{Z} / 2)$ has a basis given by $\delta_{*}\left(s^{2}\left(x_{4} y_{1}\right)\right)$. By Statement 1 , a basis for $\widetilde{H}_{*}\left(X_{1} ; \mathbb{Z} / 2\right)$ is listed in diagram (4-8) by removing $x_{1} y_{4}$. The canonical projection

$$
p: P^{7}\left(2^{r_{1}}\right) \vee P^{7}\left(2^{r_{2}}\right) \vee P^{5}\left(2^{r_{1}}\right) \vee P^{6}\left(2^{r_{1}}\right) \longrightarrow P^{6}\left(2^{r_{1}}\right)
$$

has the property that $p_{*}\left(u_{5}^{r_{1}}\right)=u_{5}^{r_{1}}, p_{*}\left(v_{6}^{r_{1}}\right)=v_{6}^{r_{1}}$ and $p_{*}(x)=0$ for $x$ to the other elements in the basis for $\widetilde{H}_{*}\left(P^{7}\left(2^{r_{1}}\right) \vee P^{7}\left(2^{r_{2}}\right) \vee P^{5}\left(2^{r_{1}}\right) \vee P^{6}\left(2^{r_{1}}\right) ; \mathbb{Z} / 2\right)$. In particular, $p_{*}\left(v_{5}^{r_{1}}\right)=0$. Note that

$$
\begin{aligned}
\mathrm{Sq}_{*}^{2} \delta_{*}\left(s^{2}\left(x_{4} y_{1}\right)\right) & =\delta_{*}\left(\mathrm{Sq}_{*}^{2}\left(s^{2}\left(x_{4} y_{1}\right)\right)\right) \\
& =\delta_{*}\left(s^{2}\left(x_{2} y_{1}\right)\right) \\
& =\delta_{*}\left(g_{1 *}\left(v_{5}^{r_{1}}\right)\right. \\
& =g_{*}^{\prime} \circ p_{*}\left(v_{5}^{r_{1}}\right) \\
& =0 .
\end{aligned}
$$


It follows that

$$
\mathrm{Sq}_{*}^{2}: H_{7}(W ; \mathbb{Z} / 2) \rightarrow H_{5}(W ; \mathbb{Z} / 2)
$$

is zero. From the exact sequence

$\pi_{6}\left(S^{5}\right)=\mathbb{Z} / 2 \stackrel{2^{r_{1}}}{\longrightarrow}{ }_{0} \pi_{6}\left(S^{5}\right)=\mathbb{Z} / 2 \longrightarrow \pi_{6}\left(P^{6}\left(2^{r_{1}}\right) \longrightarrow \pi_{5}\left(S^{5}\right)=\mathbb{Z} \stackrel{2^{r_{1}}}{\longrightarrow} \mathbb{Z}\right.$, we have

$$
\pi_{6}\left(P^{6}\left(2^{r_{1}}\right)\right)=\mathbb{Z} / 2
$$

generated by the composite

$$
\bar{\eta}: S^{6} \stackrel{\eta}{\longrightarrow} S^{5} \hookrightarrow P^{6}\left(2^{r_{1}}\right) .
$$

Thus the homotopy class $[\psi]=0$ or $\bar{\eta}$. If $[\psi]=\bar{\eta}$, then $\mathrm{Sq}^{2}: H_{7}(W ; \mathbb{Z} / 2) \rightarrow$ $H_{5}(W ; \mathbb{Z} / 2)$ is not zero, which is impossible from the above. Hence $[\psi]=0$. This finishes the proof for Statement 3.

Statement 4 There is a homotopy decomposition

$$
X \simeq P^{7}\left(2^{r_{1}}\right) \vee T_{1} \vee T_{2},
$$

where $\widetilde{H}_{*}\left(T_{1} ; \mathbb{Z} / 2\right)$ and $\widetilde{H}_{*}\left(T_{2} ; \mathbb{Z} / 2\right)$ have basis listed by the middle and the right modules in diagram (4-6), respectively.

From Statements 2 and 3, the attaching map $f \mid S_{1}^{6}$ maps into the subspace $P^{7}\left(2^{r_{2}}\right) \vee$ $P^{5}\left(2^{r_{1}}\right)$ up to homotopy because, in the range of $\pi_{6}$, we have

$$
\begin{aligned}
\pi_{6}\left(P^{7}\left(2^{r_{1}}\right) \vee P^{7}\left(2^{r_{2}}\right)\right. & \left.\vee P^{5}\left(2^{r_{1}}\right) \vee P^{6}\left(2^{r_{1}}\right)\right) \\
& \cong \pi_{6}\left(P^{7}\left(2^{r_{1}}\right)\right) \oplus \pi_{6}\left(P^{7}\left(2^{r_{2}}\right)\right) \oplus \pi_{6}\left(P^{5}\left(2^{r_{1}}\right)\right) \oplus \pi_{6}\left(P^{6}\left(2^{r_{1}}\right)\right) .
\end{aligned}
$$

Thus there is a homotopy commutative diagram of cofibre sequences

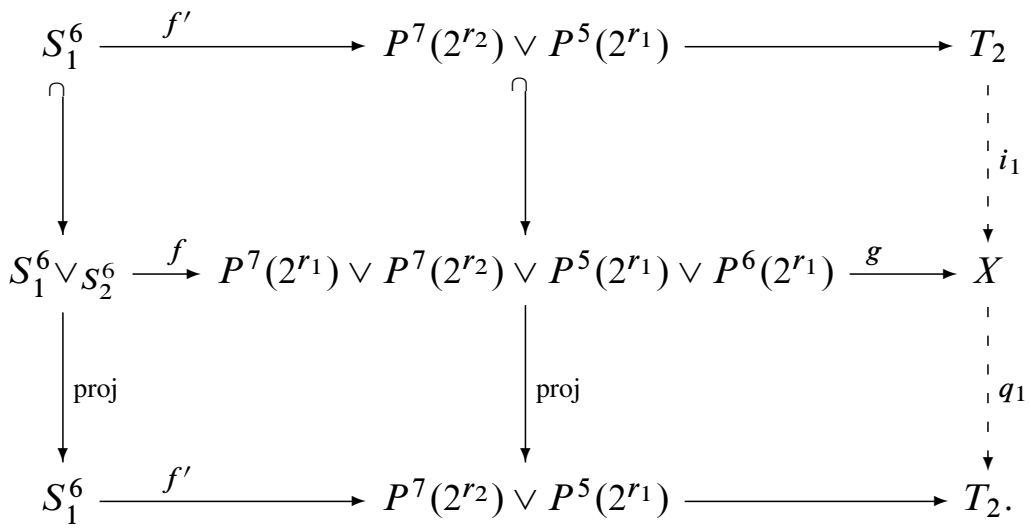


From Statement 2, there is a homotopy commutative diagram of cofibre sequences

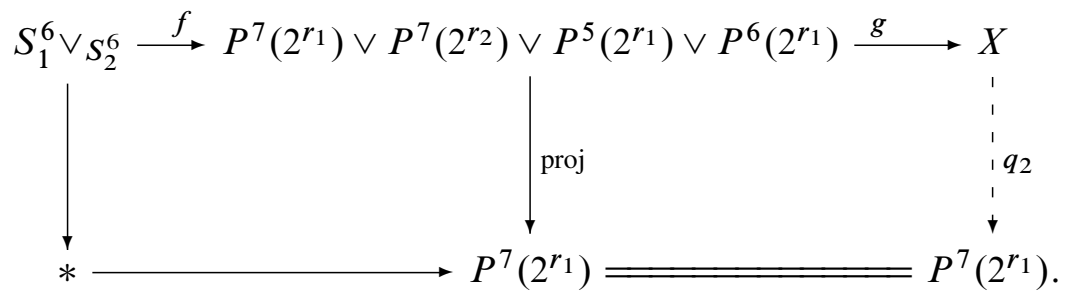

Now the composite

$$
P^{7}\left(2^{r_{1}}\right) \vee T_{2} \stackrel{\left(\left.g\right|_{\left.P^{7}\left(2^{r_{1}}\right), i_{1}\right)} ^{\longrightarrow}\right.}{\longrightarrow} X \stackrel{\left(q_{2}, q_{1}\right)}{\longrightarrow} P^{7}\left(2^{2^{r_{1}}}\right) \vee T_{2}
$$

is a homotopy equivalence by inspecting the homology and hence the statement.

Computation of the homotopy group From Statement 4, we have

$$
\begin{aligned}
\pi_{6}(X) \cong \pi_{6}\left(P^{7}\left(2^{r_{1}}\right) \vee T_{1} \vee T_{2}\right) & \cong \pi_{6}\left(P^{7}\left(2^{r_{1}}\right)\right) \oplus \pi_{6}\left(T_{1}\right) \oplus \pi_{6}\left(T_{2}\right) \\
& \cong \mathbb{Z} / 2^{r_{1}} \oplus \pi_{6}\left(T_{1}\right) \oplus \pi_{6}\left(T_{2}\right) .
\end{aligned}
$$

For computing $\pi_{6}\left(T_{1}\right)$, since $T_{1}=P^{6}\left(2^{r_{1}}\right) \cup e^{7}$, there is a right exact sequence

$$
\pi_{6}\left(S^{6}\right)=\mathbb{Z} \longrightarrow \pi_{6}\left(P^{6}\left(2^{r_{1}}\right)\right)=\mathbb{Z} / 2 \longrightarrow \pi_{6}\left(T_{1}\right),
$$

where $\pi_{6}\left(P^{6}\left(2^{r_{1}}\right)\right)=\mathbb{Z} / 2$ is given in formula (4-10). From diagram (4-6),

$$
\mathrm{Sq}_{*}^{2}: H_{7}\left(T_{1} ; \mathbb{Z} / 2\right) \longrightarrow H_{5}\left(T_{1} ; \mathbb{Z} / 2\right)
$$

is an isomorphism and so the attaching map $S^{6} \rightarrow P^{6}\left(2^{r_{1}}\right)$ of $T_{1}$ is nontrivial. It follows that $\pi_{6}\left(T_{1}\right)=0$.

Now we compute $\pi_{6}\left(T_{2}\right)$. From diagram (4-11), there is a right exact sequence

$$
\pi_{6}\left(S^{6}\right)=\mathbb{Z} \stackrel{f_{*}^{\prime}}{\rightarrow} \pi_{6}\left(P^{7}\left(2^{r_{2}}\right) \vee P^{5}\left(2^{r_{1}}\right)\right)=\pi_{6}\left(P^{7}\left(2^{r_{2}}\right)\right) \oplus \pi_{6}\left(P^{5}\left(2^{r_{1}}\right)\right) \rightarrow \pi_{6}\left(T_{2}\right) .
$$

Note that a basis for $\widetilde{H}_{*}\left(T_{2}\right)$ can be listed in the right module of diagram (4-6). The composite

$$
\pi_{6}\left(S^{6}\right)=\mathbb{Z} \stackrel{f_{*}^{\prime}}{\longrightarrow} \pi_{6}\left(P^{7}\left(2^{r_{2}}\right)\right) \oplus \pi_{6}\left(P^{5}\left(2^{r_{1}}\right)\right) \stackrel{\text { proj }}{\longrightarrow} \pi_{6}\left(P^{7}\left(2^{r_{2}}\right)\right)=\mathbb{Z} / 2^{r_{2}}
$$

is of degree $2^{r_{1}}$ because of the existence of the Bockstein $\beta_{r_{1}}$. Moreover the composite

$$
S^{6} \stackrel{f^{\prime}}{\longrightarrow} P^{7}\left(2^{r_{2}}\right) \vee P^{5}\left(2^{r_{1}}\right) \stackrel{\text { proj }}{\longrightarrow} P^{5}\left(2^{r_{1}}\right) \stackrel{\text { pinch }}{\longrightarrow} S^{5}
$$

is homotopic to $\eta$ because of the existence of the Steenrod operation $\mathrm{Sq}_{*}^{2}$. 
Case I $r_{1}=1$. According to [21, Proposition 5.1], $\pi_{6}\left(P^{5}(2)\right)=\mathbb{Z} / 4$ generated by the homotopy class of any may $S^{6} \rightarrow P^{5}(2)$ such that the composite $S^{6} \rightarrow P^{5}(2) \rightarrow S^{5}$ is $\eta$. It follows that there is a right exact sequence

$$
\mathbb{Z} \stackrel{f_{*}^{\prime}=\left(2^{r_{1}}, \lambda\right)}{\longrightarrow} \mathbb{Z} / 2^{r_{2}} \oplus \mathbb{Z} / 4 \longrightarrow \pi_{6}\left(T_{2}\right),
$$

where $\lambda: \mathbb{Z} \rightarrow \mathbb{Z} / 4$ is an epimorphism. Thus

$$
\pi_{6}\left(T_{2}\right)=\left\{\begin{array}{l}
\mathbb{Z} / 4 \text { if } r_{2}=2 \\
\mathbb{Z} / 8 \text { if } r_{2} \geq 3
\end{array}\right.
$$

Case II $r_{1}>1$. From formula (4-3), we have $\pi_{6}\left(P^{5}\left(2^{r_{1}}\right)\right)=\mathbb{Z} / 2 \oplus \mathbb{Z} / 2$. Since the composite in Equation (4-12) is essential, the composite

$$
\pi_{6}\left(S^{6}\right)=\mathbb{Z} \stackrel{f_{*}^{\prime}}{\longrightarrow} \pi_{6}\left(P^{7}\left(2^{r_{2}}\right)\right) \oplus \pi_{6}\left(P^{5}\left(2^{r_{1}}\right)\right) \stackrel{\text { proj }}{\longrightarrow} \pi_{6}\left(P^{5}\left(2^{r_{1}}\right)\right)=\mathbb{Z} / 2 \oplus \mathbb{Z} / 2
$$

is nontrivial and so there is right exact sequence

$$
\mathbb{Z} \stackrel{\left(2^{r_{1}}, \lambda\right)}{\longrightarrow} \mathbb{Z} / 2^{r_{2}} \oplus(\mathbb{Z} / 2 \oplus \mathbb{Z} / 2) \longrightarrow \pi_{6}\left(T_{2}\right)
$$

with $\lambda: \mathbb{Z} \rightarrow \mathbb{Z} / 2 \oplus \mathbb{Z} / 2$ nontrivial. It follows that

$$
\pi_{6}\left(T_{2}\right)=\mathbb{Z} / 2^{r_{1}+1} \oplus \mathbb{Z} / 2 \text { for } r_{1}>1 .
$$

The proof is finished now.

\subsection{The group $\pi_{5}\left(\Sigma^{2} K\left(\mathbb{Z} / 2^{r}, 1\right)\right)$}

We use the spectral sequence induced from Carlsson's construction for computing this group. Let $A$ be an abelian group and

$$
0 \rightarrow A_{1} \stackrel{\delta}{\rightarrow} A_{0} \rightarrow A \rightarrow 0
$$

a two-step flat resolution of $A$, ie $A_{0}$ is a free abelian group. The diagram (1-1) implies that there is a natural isomorphism

$$
\pi_{4}\left(\Sigma^{2} K(A, 1)\right) \simeq A \widetilde{\otimes} A,
$$

where

$$
\widetilde{\otimes}^{2}(A)=A \widetilde{\otimes} A:=A \otimes A /(a \otimes b+b \otimes a, a, b \in A) .
$$


Given a free abelian group $\bar{A}$, Theorem 2.5 (2) implies the following natural exact sequence:

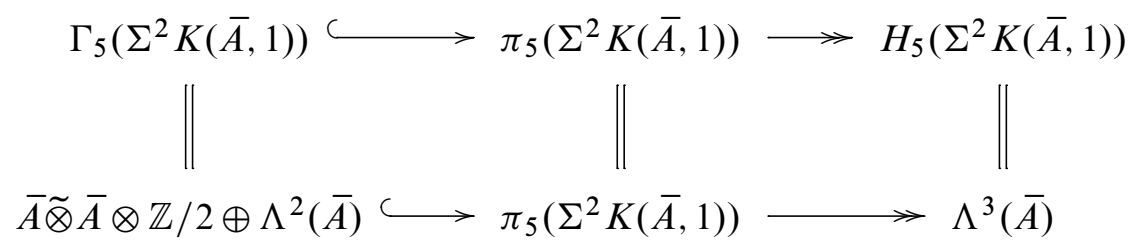

The spectral sequence (2-9) for $n=2$, gives the following diagram of exact sequences:

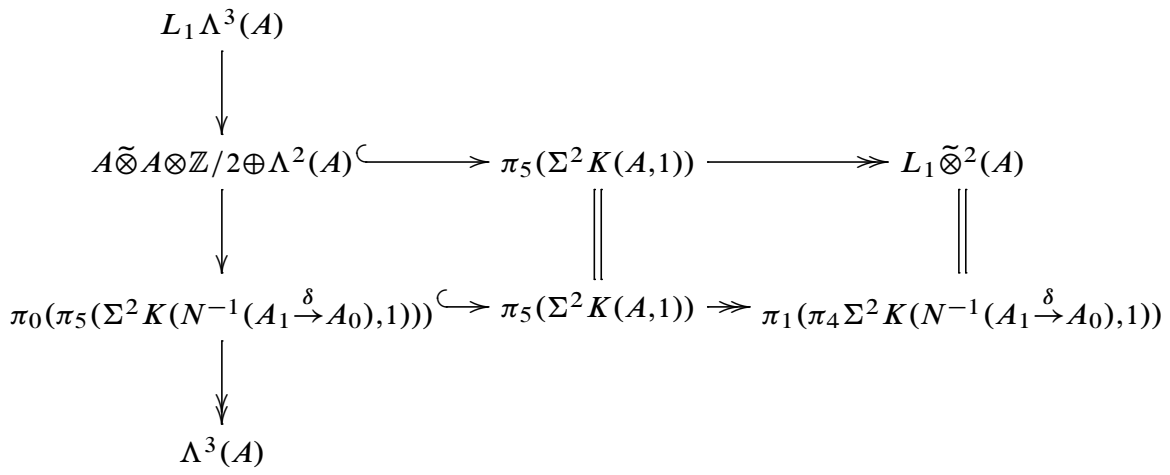

Consider the first derived functor of the functor $\tilde{\otimes}^{2}$. The short exact sequence

$$
L S P^{2}(A) \rightarrow L \otimes^{2}(A) \rightarrow L \widetilde{\otimes}^{2}(A)
$$

in the derived category has the following model:

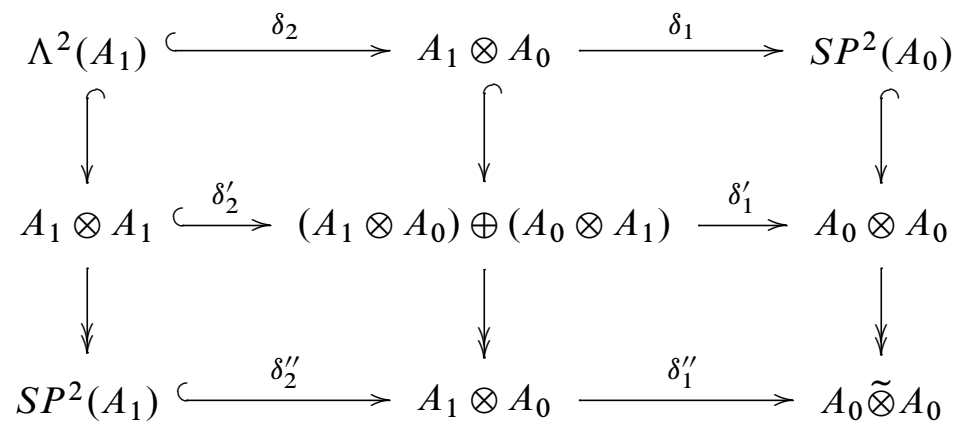


with

$$
\begin{aligned}
\delta_{2}\left(a_{1} \wedge a_{1}^{\prime}\right) & =a_{1} \otimes \delta\left(a_{1}^{\prime}\right)-a_{1}^{\prime} \otimes \delta\left(a_{1}\right) \\
\delta_{1}\left(a_{1} \otimes a_{0}\right) & =a_{0} \delta\left(a_{1}\right) \\
\delta_{2}^{\prime}\left(a_{1} \otimes a_{1}^{\prime}\right) & =\left(a_{1} \otimes \delta\left(a_{1}^{\prime}\right),-a_{1}^{\prime} \otimes \delta\left(a_{1}\right)\right) \\
\delta_{1}^{\prime}\left(a_{1} \otimes a_{0}, a_{1}^{\prime} \otimes a_{0}^{\prime}\right) & =\delta\left(a_{1}\right) \otimes a_{0}+\delta\left(a_{1}^{\prime}\right) \otimes a_{0}^{\prime} \\
\delta_{2}^{\prime \prime}\left(a_{1} a_{1}^{\prime}\right) & =a_{1} \otimes \delta\left(a_{1}^{\prime}\right)+a_{1}^{\prime} \otimes \delta\left(a_{1}\right) \\
\delta_{1}^{\prime \prime}\left(a_{1} \otimes a_{0}\right) & =\partial\left(a_{1}\right) \widetilde{\otimes} a_{0}
\end{aligned}
$$

for $a_{0}, a_{0}^{\prime} \in A_{0}, a_{1}, a_{1}^{\prime} \in A_{1}$. For $n \geq 2$, looking at the resolution $\mathbb{Z} \stackrel{n}{\rightarrow} \mathbb{Z}$ of the cyclic group $\mathbb{Z} / n$, we obtain the following representative of the element $L \widetilde{\otimes}^{2}(\mathbb{Z} / n)$ in the derived category:

$$
\mathbb{Z} \stackrel{2 n}{\rightarrow} \mathbb{Z} \stackrel{n}{\rightarrow} \mathbb{Z} / 2
$$

In particular,

$$
L_{1} \widetilde{\otimes}^{2}\left(\mathbb{Z} / 2^{k}\right)=\mathbb{Z} / 2^{k+1}, \quad k \geq 1 .
$$

Here $L_{1} \widetilde{\otimes}^{2}$ denotes the first derived functor of $\widetilde{\otimes}^{2}$ (see Section 2.2).

We will use the following:

Lemma 4.17 [20, Lemma 2.1] Let $G_{*}$ be a simplicial group and let $n \geq 0$. Suppose that $\pi_{0}\left(G_{*}\right)$ acts trivially on $\pi_{n}\left(G_{*}\right)$. Then the homotopy group $\pi_{n}\left(G_{*}\right)$ is contained in the center of $G_{n} / \mathcal{B} G_{n}$, where $\mathcal{B} G_{n}$ is the $n$-th simplicial boundary subgroup of $G_{n}$.

Theorem 4.18 The homotopy group

$$
\pi_{5}\left(\Sigma^{2} K\left(\mathbb{Z} / 2^{r}, 1\right)\right)= \begin{cases}\mathbb{Z} / 8 & \text { if } r=1, \\ \mathbb{Z} / 2^{r+1} \oplus \mathbb{Z} / 2 & \text { if } r>1 .\end{cases}
$$

Proof The proof splits into two cases.

Case $1 r=1$. The natural epimorphism $\mathbb{Z} \rightarrow \mathbb{Z} / 2$ induces the homomorphisms

$$
\pi_{n}\left(S^{3}\right)=\pi_{n}\left(\Sigma^{2} K(\mathbb{Z}, 1)\right) \rightarrow \pi_{n}\left(\Sigma^{2} K(\mathbb{Z} / 2,1)\right)=\pi_{n}\left(\Sigma^{2} \mathbb{R} P^{\infty}\right), \quad n \geq 1 .
$$

The diagram (4-15) together with (4-16) implies the following short exact sequences:

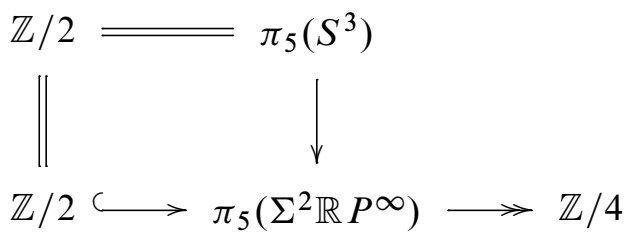


Consider this map simplicially, at the level of the natural map between the Carlsson constructions $F\left(S^{2}\right)=F^{\mathbb{Z}}\left(S^{2}\right) \rightarrow F^{\mathbb{Z} / 2}\left(S^{2}\right)$ :

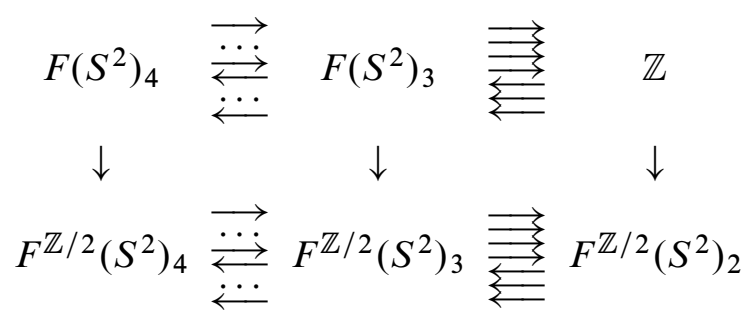

Here $F^{\mathbb{Z} / 2}\left(S^{2}\right)_{k}$ is the free product of $\left(\begin{array}{l}k \\ 2\end{array}\right)$ copies of $\mathbb{Z} / 2$. In particular

$$
F^{\mathbb{Z} / 2}\left(S^{2}\right)_{4}=\left\langle s_{j} s_{i}(\sigma) 0 \leq i<j \leq 3 \mid\left(s_{j} s_{i}(\sigma)\right)^{2}=1\right\rangle .
$$

Using the description of the element (2-7), we see that the simplicial cycle which defines the image of $\pi_{5}\left(S^{3}\right)$ in $\pi_{5}\left(\Sigma^{2} \mathbb{R} P^{\infty}\right)$ can be chosen of the form

$$
\left[\left[s_{2} s_{1}(\sigma), s_{1} s_{0}(\sigma)\right],\left[s_{2} s_{1}(\sigma), s_{2} s_{0}(\sigma)\right]\right] \in F^{\mathbb{Z} / 2}\left(S^{2}\right)_{4}
$$

With the help of Lemma 4.17, we have

$$
\begin{array}{r}
{\left[\left[s_{2} s_{1}(\sigma), s_{1} s_{0}(\sigma)\right],\left[s_{2} s_{1}(\sigma), s_{2} s_{0}(\sigma)\right]\right]=\left[\left[\left(s_{2} s_{1}(\sigma), s_{1} s_{0}(\sigma)\right],\left(s_{2} s_{1}(\sigma) s_{2} s_{0}(\sigma)\right)^{2}\right]\right.} \\
\equiv\left[\left[s_{2} s_{1}(\sigma), s_{1} s_{0}(\sigma)\right],\left(s_{2} s_{1}(\sigma), s_{2} s_{0}(\sigma)\right)\right]^{2} \quad \bmod \mathcal{B}^{\mathbb{Z} / 2}\left(S^{2}\right)_{4}
\end{array}
$$

since $\left[\left[s_{2} s_{1}(\sigma), s_{1} s_{0}(\sigma)\right], s_{2} s_{1}(\sigma) s_{2} s_{0}(\sigma)\right]$ is a cycle in $F^{\mathbb{Z} / 2}\left(S^{2}\right)$. That is, the image of the element $\pi_{5}\left(S^{3}\right)$ is divisible by 2 in $\pi_{5}\left(\Sigma^{2} \mathbb{R} P^{\infty}\right)$. The diagram (4-17) implies the result.

Case $2 r>1$. Now the diagram (4-15) together with (4-16) implies the following short exact sequence

$$
0 \rightarrow \mathbb{Z} / 2 \rightarrow \pi_{5}\left(\Sigma^{2} K\left(\mathbb{Z} / 2^{r}, 1\right)\right) \rightarrow \mathbb{Z} / 2^{r+1} \rightarrow 0
$$

Therefore, $\pi_{5}\left(\Sigma^{2} K\left(\mathbb{Z} / 2^{r}, 1\right)\right)$ is either $\mathbb{Z} / 2^{r+2}$ or $\mathbb{Z} / 2^{r+1} \oplus \mathbb{Z} / 2$. 
By Theorem 2.5 (2), the Whitehead exact sequence for $\Sigma^{2} K(A, 1)$ has the following form:

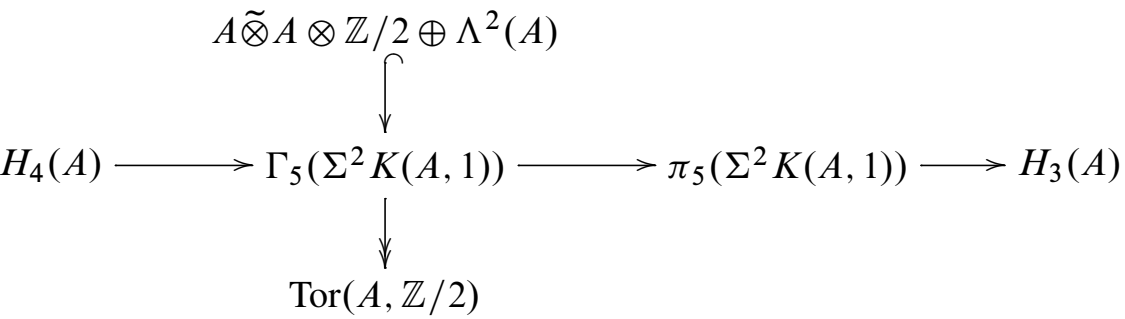

For $A=\mathbb{Z} / 2^{r}$ it is of the following form:

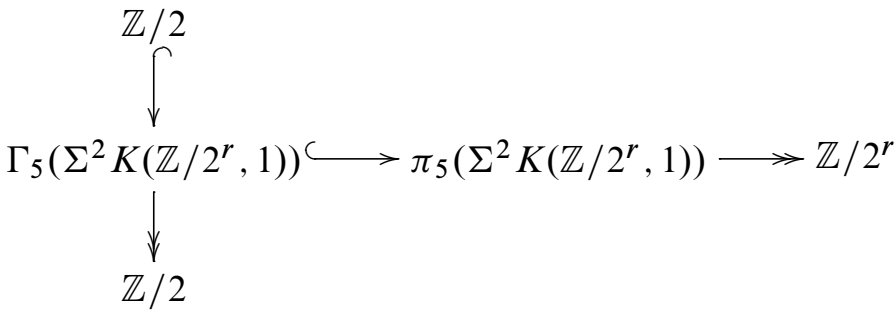

The natural projection $\mathbb{Z} / 2^{r} \rightarrow \mathbb{Z} / 2$ induces the map

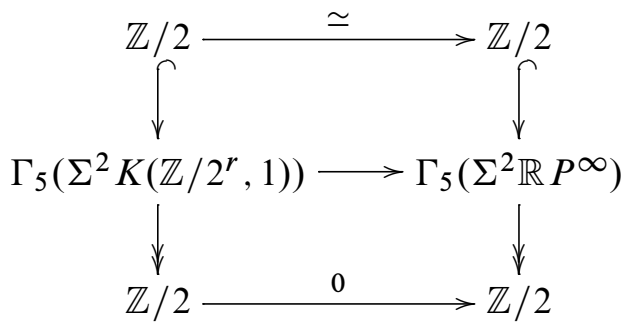

where the lower map is zero since the induced map $\operatorname{Tor}\left(\mathbb{Z} / 2^{r}, \mathbb{Z} / 2\right) \rightarrow \operatorname{Tor}(\mathbb{Z} / 2, \mathbb{Z} / 2)$ is zero. The fact that $\pi_{5}\left(\Sigma^{2} \mathbb{R} P^{\infty}\right)=\mathbb{Z} / 8$ together with diagram (4-19) implies that $\Gamma_{5}\left(\Sigma^{2} \mathbb{R} P^{\infty}\right)=\mathbb{Z} / 4$. Hence $\Gamma_{5}\left(\Sigma^{2} K\left(\mathbb{Z} / 2^{r}, 1\right)\right)=\mathbb{Z} / 2 \oplus \mathbb{Z} / 2$, since there is no any endomorphism $\mathbb{Z} / 4 \rightarrow \mathbb{Z} / 4$ with zero map on quotients $\mathbb{Z} / 2 \rightarrow \mathbb{Z} / 2$ (as in diagram (4-21)). The diagram (4-20) and exact sequence (4-18) implies that $\pi_{5}\left(\Sigma^{2} K\left(\mathbb{Z} / 2^{r}, 1\right)\right)=\mathbb{Z} / 2^{r+1} \oplus \mathbb{Z} / 2$.

\subsection{Applications}

Proposition 4.19 The group $\Gamma_{5}\left(\Sigma \mathbb{R} P^{\infty}\right)=\mathbb{Z} / 2 \oplus \mathbb{Z} / 2 \oplus \mathbb{Z} / 2$. 
Proof Consider the Whitehead exact sequence

$$
\begin{aligned}
\pi_{6}\left(\Sigma \mathbb{R} \mathrm{P}^{\infty}\right) \stackrel{h}{\longrightarrow}{ }_{0} H_{6}\left(\Sigma \mathbb{R} \mathrm{P}^{\infty}\right)=\mathbb{Z} / 2 \longrightarrow \Gamma_{5}\left(\Sigma \mathbb{R} \mathrm{P}^{\infty}\right) \\
\longrightarrow \pi_{5}\left(\Sigma \mathbb{R} \mathrm{P}^{\infty}\right)=\mathbb{Z} / 2 \oplus \mathbb{Z} / 2 \longrightarrow H_{5}\left(\Sigma \mathbb{R} \mathrm{P}^{\infty}\right)=0,
\end{aligned}
$$

where $\pi_{5}\left(\Sigma \mathbb{R} \mathrm{P}^{\infty}\right)=\mathbb{Z} / 2 \oplus \mathbb{Z} / 2$ by Theorem 4.10 and the Hurewicz homomorphism

$$
h_{6}: \pi_{6}\left(\Sigma \mathbb{R} \mathrm{P}^{\infty}\right) \rightarrow H_{6}\left(\Sigma \mathbb{R} \mathrm{P}^{\infty}\right)
$$

is zero because, otherwise, it would induces a splitting of $\Sigma \mathbb{R} \mathrm{P}^{5}$ which is impossible by inspecting the Steenrod operation on mod 2 homology. Thus the order of $\Gamma_{5}\left(\Sigma \mathbb{R} \mathrm{P}^{\infty}\right)$ is 8 . We have to determine the group $\Gamma_{5}\left(\Sigma \mathbb{R} \mathrm{P}^{\infty}\right)$. By the definition,

$$
\Gamma_{5}\left(\Sigma \mathbb{R} \mathrm{P}^{\infty}\right)=\operatorname{Im}\left(\pi_{5}\left(\Sigma \mathbb{R} \mathrm{P}^{3}\right) \rightarrow \pi_{5}\left(\Sigma \mathbb{R} \mathrm{P}^{4}\right)\right) .
$$

Thus the inclusion $\Sigma \mathbb{R} \mathrm{P}^{3} \rightarrow \Sigma \mathbb{R} \mathrm{P}^{\infty}$ induces an epimorphism

$$
\Gamma_{5}\left(\Sigma \mathbb{R} \mathrm{P}^{3}\right) \longrightarrow \Gamma_{5}\left(\Sigma \mathbb{R} \mathrm{P}^{\infty}\right) .
$$

Note that $\mathbb{R P}^{3}=\mathrm{SO}(3)$ and so, by the Hopf fibration,

$$
\begin{aligned}
\pi_{5}(\Sigma \mathrm{SO}(3)) & \cong \pi_{5}(B \mathrm{SO}(3)) \oplus \pi_{5}(\Sigma \mathrm{SO}(3) \wedge \mathrm{SO}(3)) \\
& \cong \pi_{4}(\mathrm{SO}(3)) \oplus \pi_{5}\left(\Sigma \mathbb{R} \mathrm{P}^{3} \wedge \mathbb{R} \mathrm{P}^{3}\right) \\
& \cong \mathbb{Z} / 2 \oplus \pi_{5}\left(\Sigma \mathbb{R} \mathrm{P}^{3} \wedge \mathbb{R}^{3}\right)
\end{aligned}
$$

From Lemmas 4.12 and 4.13,

$$
\begin{aligned}
\pi_{5}\left(\Sigma \mathbb{R} \mathrm{P}^{3} \wedge \mathbb{R} \mathrm{P}^{3}\right) & \cong \Gamma_{5}\left(\Sigma \mathbb{R} \mathrm{P}^{3} \wedge \mathbb{R} \mathrm{P}^{3}\right) \oplus \mathbb{Z} / 2 \oplus \mathbb{Z} / 2 \\
& \cong \pi_{5}\left(\Sigma \mathbb{R} \mathrm{P}^{2} \wedge \mathbb{R} \mathrm{P}^{2}\right) \oplus \mathbb{Z} / 2 \oplus \mathbb{Z} / 2 \\
& \cong \mathbb{Z} / 2 \oplus \mathbb{Z} / 2 \oplus \mathbb{Z} / 2 \oplus \mathbb{Z} / 2
\end{aligned}
$$

It follows that its quotient $\Gamma_{5}\left(\Sigma \mathbb{R} \mathrm{P}^{\infty}\right)$ must be an elementary 2 -group and so hence the result.

Proposition 4.20 For the suspended projective spaces,

$$
\pi_{5}\left(\Sigma \mathbb{R} P^{n}\right)= \begin{cases}\mathbb{Z} / 2 & \text { if } n=1, \\ \mathbb{Z} / 2^{\oplus 3} & \text { if } n=2, \\ \mathbb{Z} / 2^{\oplus 5} & \text { if } n=3, \\ \mathbb{Z} / 2^{\oplus 3} & \text { if } n=4, \\ \mathbb{Z} / 2^{\oplus 2} & \text { if } 3 \leq n \leq \infty .\end{cases}
$$


Proof When $n=1, \pi_{5}\left(S^{2}\right)=\mathbb{Z} / 2$ from Toda's table [15]. When $n=2, \pi_{5}\left(\Sigma \mathbb{R} \mathrm{P}^{2}\right)=$ $\mathbb{Z} / 2^{\oplus 3}$ is given in [21, Theorem 6.36]. When $n=3, \pi_{5}\left(\Sigma \mathbb{R} \mathrm{P}^{3}\right)$ has been computed in Proposition 4.19. For $n>4$, since $\operatorname{sk}_{6}\left(\Sigma \mathbb{R} \mathrm{P}^{\infty}\right)=\Sigma \mathbb{R} \mathrm{P}^{5}$,

$$
\pi_{5}\left(\Sigma \mathbb{R} \mathrm{P}^{n}\right) \cong \pi_{5}\left(\Sigma \mathbb{R} \mathrm{P}^{\infty}\right)=\mathbb{Z} / 2 \oplus \mathbb{Z} / 2
$$

by Theorem 4.14. The remaining case is $\pi_{5}\left(\Sigma \mathbb{R} \mathrm{P}^{4}\right)$. Let $F$ be the homotopy fibre of the pinch map $\Sigma \mathbb{R} \mathrm{P}^{6} \longrightarrow \Sigma \mathbb{R} \mathrm{P}^{6} / \mathbb{R} \mathrm{P}^{4}=M(\mathbb{Z} / 2,6)$. By inspecting the Serre spectral sequence to the fibre sequence

$$
\Omega M(\mathbb{Z} / 2,6) \longrightarrow F \longrightarrow \Omega \Sigma \mathbb{R} \mathrm{P}^{6}
$$

the canonical injection $j: \Sigma \mathbb{R} \mathrm{P}^{4} \rightarrow F$ induces an isomorphism on $H_{k}(; \mathbb{Z} / 2)$ for $k \leq 6$ and so

$$
j_{*}: \pi_{k}\left(\Sigma \mathbb{R} \mathrm{P}^{4}\right) \longrightarrow \pi_{k}(F)
$$

is an isomorphism for $k \leq 5$. In particular, $\pi_{5}\left(\Sigma \mathbb{R} \mathrm{P}^{4}\right) \cong \pi_{5}(F)$. From the exact sequence

$$
\pi_{5}(\Omega M(\mathbb{Z} / 2,6))=\mathbb{Z} / 2 \longrightarrow \pi_{5}(F) \longrightarrow \pi_{5}\left(\Sigma \mathbb{R} \mathrm{P}^{6}\right)=\mathbb{Z} / 2 \oplus \mathbb{Z} / 2,
$$

the group $\pi_{5}(F)$ is of order at most 8 and so is $\pi_{5}\left(\Sigma \mathbb{R} \mathrm{P}^{4}\right)$. From Proposition 4.19,

$$
\Gamma_{5}\left(\Sigma \mathbb{R} \mathrm{P}^{\infty}\right)=\operatorname{Im}\left(\pi_{5}\left(\Sigma \mathbb{R} \mathrm{P}^{3}\right) \rightarrow \pi_{5}\left(\Sigma \mathbb{R} \mathrm{P}^{4}\right)\right)=\mathbb{Z} / 2^{\oplus 3}
$$

It follows that $\pi_{5}\left(\Sigma \mathbb{R} \mathrm{P}^{4}\right)=\mathbb{Z} / 2^{\oplus 3}$ and hence the result follows.

Proposition $4.21 \pi_{5}\left(\Sigma K\left(\Sigma_{3}, 1\right)\right) \simeq \mathbb{Z} / 2 \oplus \mathbb{Z} / 2$.

Proof This follows from the analysis of the map between the Whitehead exact sequences (2-2) induced by the natural map $\mathbb{Z} / 2 \hookrightarrow \Sigma_{3}$ :

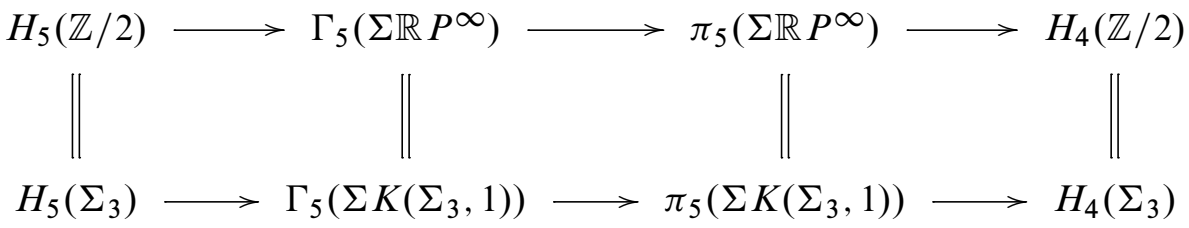


Here the natural isomorphism $\Gamma_{5}\left(\Sigma \mathbb{R} P^{\infty}\right) \rightarrow \Gamma_{5}\left(\Sigma K\left(\Sigma_{3}, 1\right)\right)$ follows from the diagram:

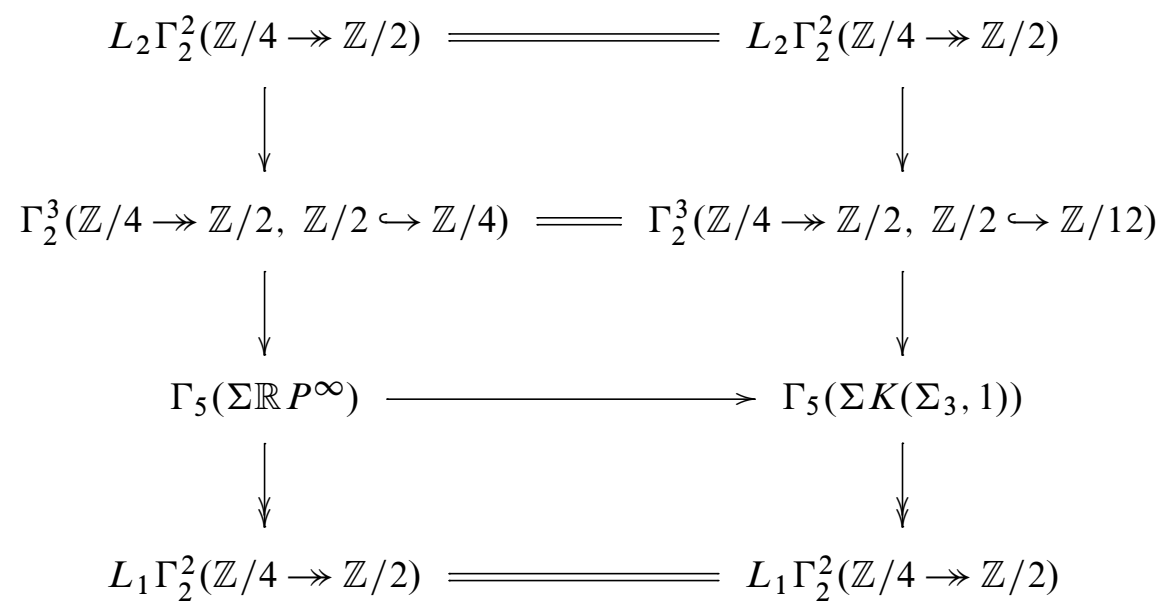

This completes the proof.

\section{Relation to $K$-theory}

As we mentioned in the introduction, there is a natural relation between the problem considered and algebraic $K$-theory. Since the plus-construction $K(G, 1) \rightarrow K(G, 1)^{+}$ is a homological equivalence, there is a natural weak homotopy equivalence

$$
\Sigma K(G, 1) \rightarrow \Sigma\left(K(G, 1)^{+}\right)
$$

This defines the natural suspension map

$$
\pi_{n}\left(K(G, 1)^{+}\right) \rightarrow \pi_{n+1}\left(\Sigma\left(K(G, 1)^{+}\right)\right)=\pi_{n+1}(\Sigma K(G, 1))
$$

for $n \geq 1$.

Given a group $G$ and its maximal perfect normal subgroup $P \triangleleft G$, one has natural isomorphism $\pi_{n}\left(K(P, 1)^{+}\right) \simeq \pi_{n}\left(K(G, 1)^{+}\right), n \geq 2$ since $K(P, 1)^{+}$is homotopy equivalent to the universal covering space of $K(G, 1)^{+}$. 
For a perfect group $G$, the Whitehead exact sequences form the following commutative diagram:

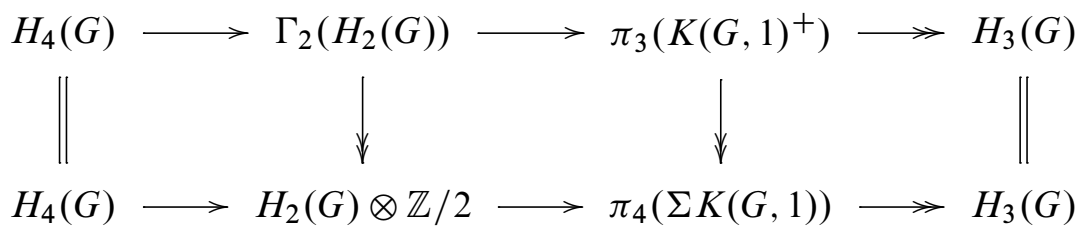

Here we will look at the applications of the following two classical constructions:

(1) Let $R$ be a ring and $G=E(R)$, the group generated by elementary matrices. The group $E(R)$ is perfect and the plus-construction $K(E(R), 1)^{+}$also denoted $\widetilde{K}(R)$, defines the algebraic $K$-theory of $R: K_{n}(R)=\pi_{n}\left(K(E(R), 1)^{+}\right), n \geq 2$.

(2) Let $\Sigma_{\infty}$ be the infinite permutation groups and $A_{\infty}$ is the infinite alternating subgroup. There is the following description of stable homotopy groups of spheres [13]:

$$
\pi_{n}^{S}=\pi_{n}\left(K\left(\Sigma_{\infty}, 1\right)^{+}\right)=\pi_{n}\left(K\left(A_{\infty}, 1\right)^{+}\right), n \geq 2 .
$$

\subsection{Computation for the group $E(R)$}

Let $R$ be a ring. In this case, one has the natural homomorphisms:

$$
K_{n}(R) \rightarrow \pi_{n+1}(\Sigma K(E(R), 1)), \quad n \geq 2 .
$$

For $n=2$, clearly one has the natural isomorphism

$$
K_{2}(R) \simeq H_{2}(E(R)) \simeq \pi_{3}(\Sigma K(E(R), 1)) .
$$

It is shown in [1] that the map $\Gamma_{2}\left(K_{2}(R)\right) \rightarrow K_{3}(R)$ factors as

$$
\Gamma_{2}\left(K_{2}(R)\right) \rightarrow K_{2}(R) \otimes K_{1}(\mathbb{Z}) \stackrel{\star}{\rightarrow} K_{3}(R),
$$

where $\star$ is the product in algebraic $K$-theory: $\star: K_{i}(S) \otimes K_{j}(T) \rightarrow K_{i+j}(S \otimes T)$. Hence the diagram (5-1) has the following form:

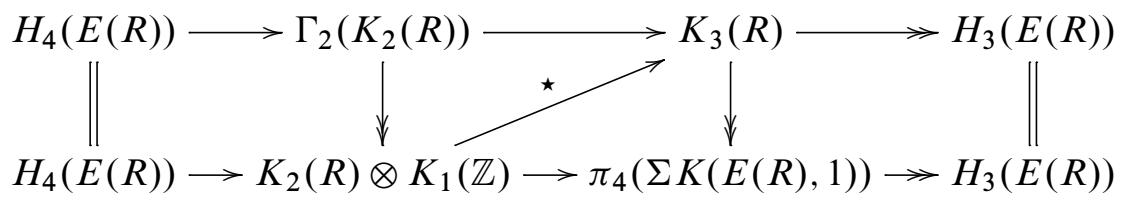

and the natural map

$$
K_{3}(R) \rightarrow \pi_{4}(\Sigma K(E(R), 1))
$$


is an isomorphism. From Equations (5-3) and (5-5) together with the fact that $\operatorname{SL}(\mathbb{Z})=$ $E(\mathbb{Z})$, we have the following:

Theorem 5.1 The natural homomorphism

$$
K_{n}(R) \longrightarrow \pi_{n+1}(\Sigma K(E(R), 1))
$$

is an isomorphism for $n=2,3$. In particular,

$$
\begin{aligned}
& \pi_{3}(\Sigma K(\operatorname{SL}(\mathbb{Z}), 1)) \cong K_{2}(\mathbb{Z}) \cong \mathbb{Z} / 2, \\
& \pi_{4}(\Sigma K(\operatorname{SL}(\mathbb{Z}), 1)) \cong K_{3}(\mathbb{Z}) \cong \mathbb{Z} / 48 .
\end{aligned}
$$

Remark 5.2 The isomorphism (5-5) and Carlsson construction $F^{E(R)}\left(S^{1}\right)$ gives a way, for an element of $K_{3}(R)$, to associate an element from $F^{E(R)}\left(S^{1}\right)_{3}=E(R) *$ $E(R) * E(R)$ (uniquely modulo $\mathcal{B} F^{E(R)}\left(S^{1}\right)$ ):

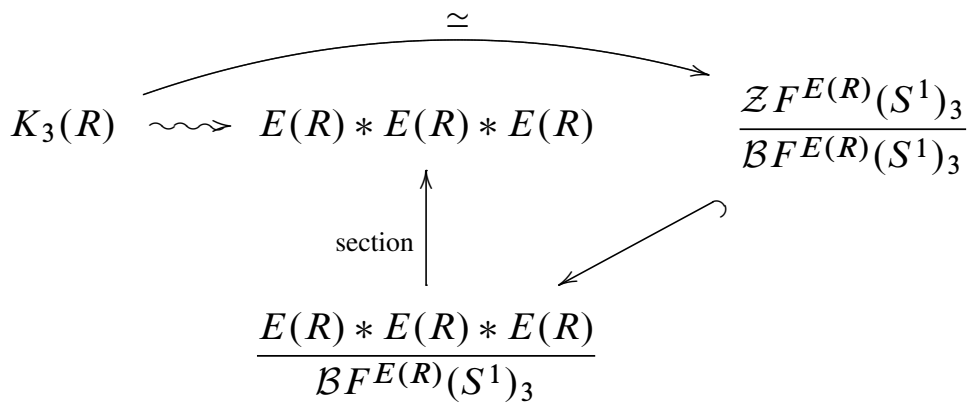

It is interesting to represent in this way known elements from $K_{3}(R)$ for rings. For $R=\mathbb{Z}, x \in \mathrm{SL}(\mathbb{Z})=E(\mathbb{Z})$, denote by $x^{(1)}, x^{(2)}, x^{(3)}$ the correspondent elements in the free cube $\mathrm{SL}(\mathbb{Z}) * \mathrm{SL}(\mathbb{Z}) * \mathrm{SL}(\mathbb{Z})$. Take the following commuting elements of $\operatorname{SL}(\mathbb{Z})$ :

$$
u=\left(\begin{array}{ccc}
1 & 0 & 0 \\
0 & -1 & 0 \\
0 & 0 & 1
\end{array}\right), \quad v=\left(\begin{array}{ccc}
1 & 0 & 0 \\
0 & 1 & 0 \\
0 & 0 & -1
\end{array}\right)
$$

The structure of the element (2-7), diagram (5-4) and well-known facts about structure of $K_{2}(\mathbb{Z})$ imply that, using the above notation, the element

$$
\left[\left[u^{(2)}, v^{(3)}\right],\left[u^{(1)}, v^{(3)}\right]\right]
$$

corresponds to the element of order 2 in $K_{3}(\mathbb{Z})$. It would be interesting to see an element of $\operatorname{SL}(\mathbb{Z}) * \operatorname{SL}(\mathbb{Z}) * \operatorname{SL}(\mathbb{Z})$ which corresponds to the generator of $K_{3}(\mathbb{Z})=\mathbb{Z} / 48$. 
Consider the case $R=\mathbb{Z}$ and $n=5$. In this case, $E(\mathbb{Z})=\operatorname{SL}(\mathbb{Z})$ and we have the following commutative diagram with exact horizontal sequences:

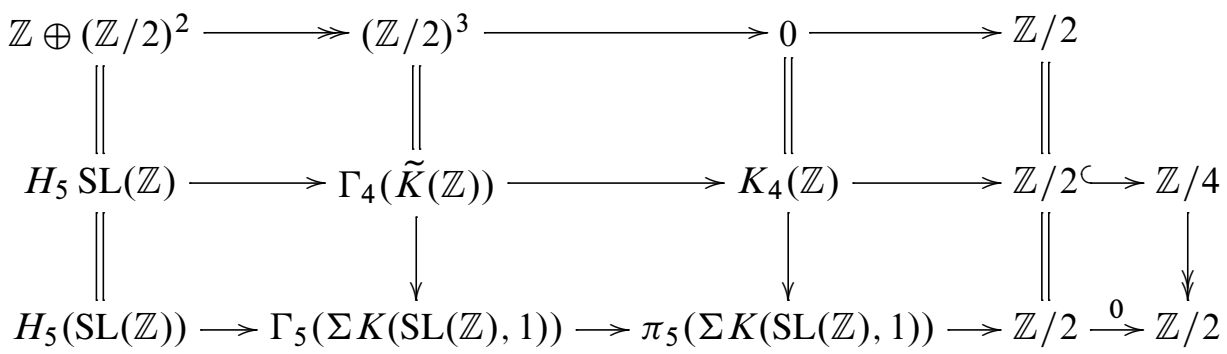

and the following commutative diagram:

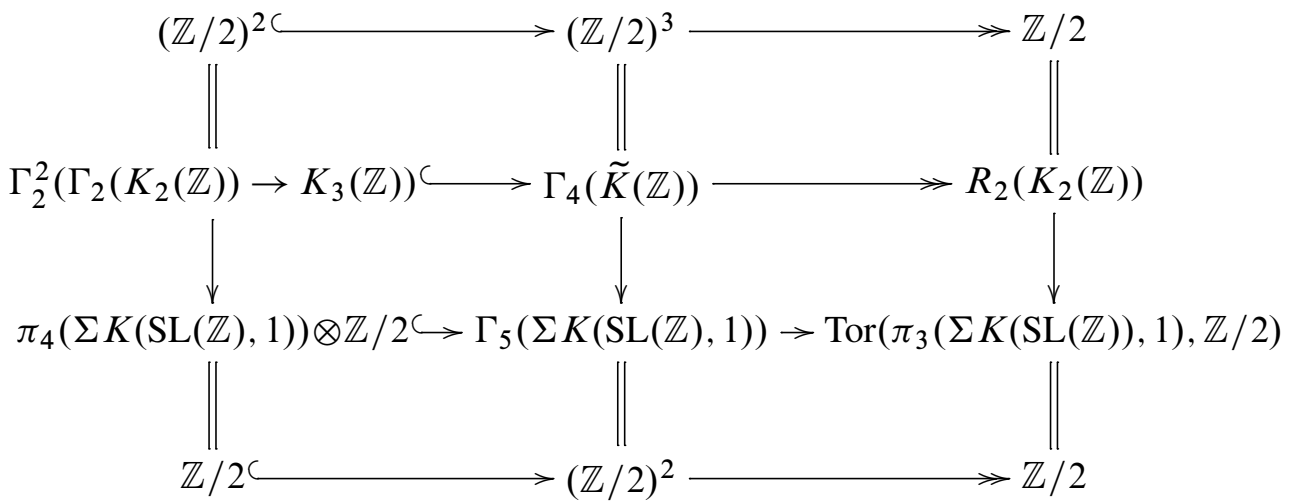

Simple analysis shows that the suspension map $\Gamma_{4}(\tilde{K}(\mathbb{Z})) \rightarrow \Gamma_{5}(\Sigma K(\operatorname{SL}(\mathbb{Z}), 1))$ is an epimorphism and therefore we have the following theorem:

Theorem 5.3 The Hurewicz homomorphism

$$
\pi_{5}(\Sigma K(\mathrm{SL}(\mathbb{Z}), 1)) \rightarrow H_{4}(\mathrm{SL}(\mathbb{Z}))=\mathbb{Z} / 2
$$

is an isomorphism.

Remark Since $K_{4}(\mathbb{Z})=0$, we see that the natural homomorphism

$$
K_{4}(\mathbb{Z}) \rightarrow \pi_{5}(\Sigma K(\mathrm{SL}(\mathbb{Z}), 1))
$$

is not an isomorphism.

\subsection{Computation for the group $A_{4}$}

Here we will use (5-2) for certain computations. 
Theorem 5.4 Let $A_{4}$ be the 4-th alternating group. Then $\pi_{4}\left(\Sigma K\left(A_{4}, 1\right)\right)=\mathbb{Z} / 4$.

Proof First recall that ${ }^{3}$

$$
\begin{aligned}
& H_{1}\left(A_{4}\right)=\mathbb{Z} / 3, \quad H_{2}\left(A_{4}\right)=\mathbb{Z} / 2, \quad H_{3}\left(A_{4}\right)=\mathbb{Z} / 6, \quad H_{4}\left(A_{4}\right)=0, \\
& H_{2}\left(A_{\infty}\right)=\mathbb{Z} / 6, \quad H_{3}\left(A_{\infty}\right)=\mathbb{Z} / 12, \quad \pi_{3}\left(\Sigma K\left(A_{4}, 1\right)\right)=\mathbb{Z} / 6
\end{aligned}
$$

Consider the Whitehead exact sequence for the space $\Sigma K\left(A_{4}, 1\right)$ :

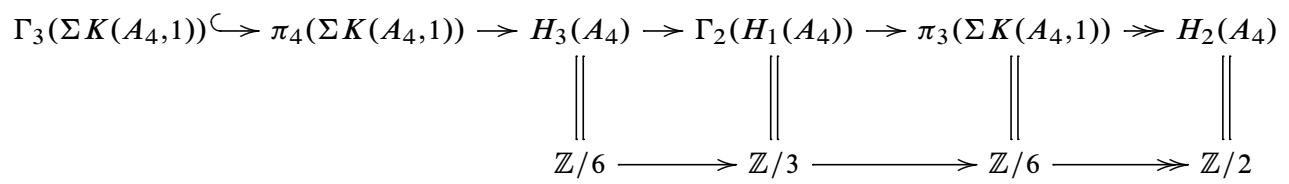

Since $R_{2}\left(\pi_{2} \Sigma K\left(A_{4}, 1\right)\right)=R_{2}(\mathbb{Z} / 3)=0$, we have

$$
\Gamma_{3}\left(\Sigma K\left(A_{4}, 1\right)\right)=\Gamma_{2}^{2}(\mathbb{Z} / 3 \hookrightarrow \mathbb{Z} / 6) .
$$

It follows from the definition of the functor $\Gamma_{2}^{2}$ that it is isomorphic to the pushout:

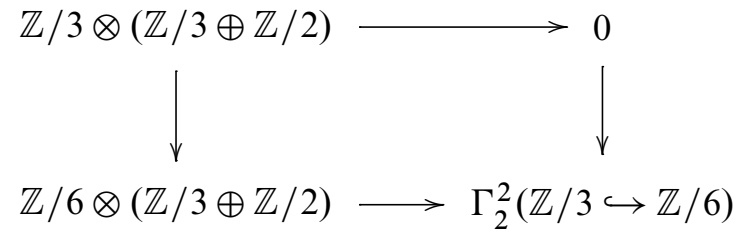

That is, $\Gamma_{2}^{2}(\mathbb{Z} / 3 \hookrightarrow \mathbb{Z} / 6)=\mathbb{Z} / 2$ and there is the following short exact sequence:

$$
0 \rightarrow \mathbb{Z} / 2 \rightarrow \pi_{4}\left(\Sigma K\left(A_{4}, 1\right)\right) \rightarrow \mathbb{Z} / 2 \rightarrow 0
$$

We come to the extension problem: is it $\mathbb{Z} / 2 \oplus \mathbb{Z} / 2$ or $\mathbb{Z} / 4$ ?

Consider the monomorphism $A_{4} \hookrightarrow A_{\infty}$ and the map between corresponding Whitehead sequences:

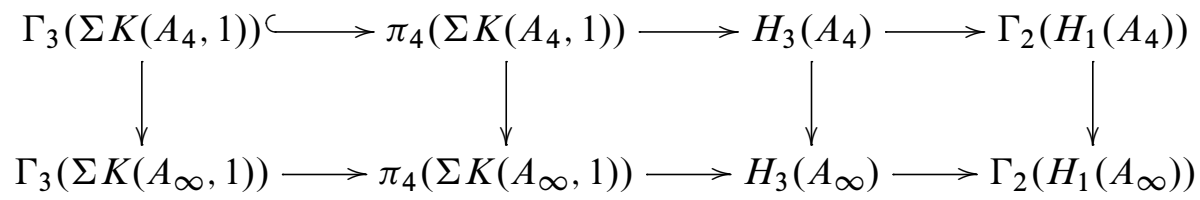

\footnotetext{
${ }^{3}$ These computations were done with the help of HAP-system. The authors thank Graham Ellis for these computations
} 
which is

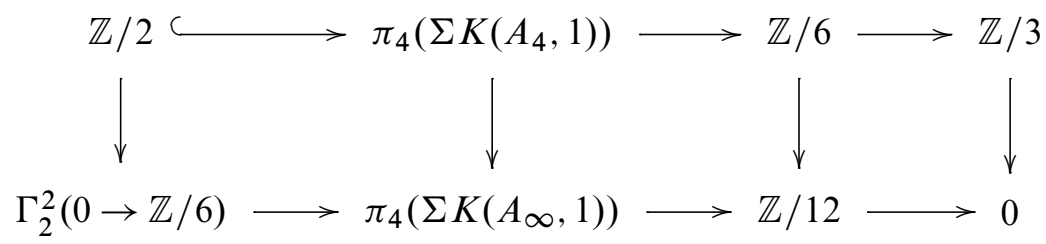

It is easy to see that $\Gamma_{2}^{2}(0 \rightarrow \mathbb{Z} / 6)=\mathbb{Z} / 2$ and that $\Gamma_{2}^{2}(\mathbb{Z} / 3 \hookrightarrow \mathbb{Z} / 6) \rightarrow \Gamma_{2}^{2}(0 \rightarrow \mathbb{Z} / 6)$ is an isomorphism. We obtain the following diagram:

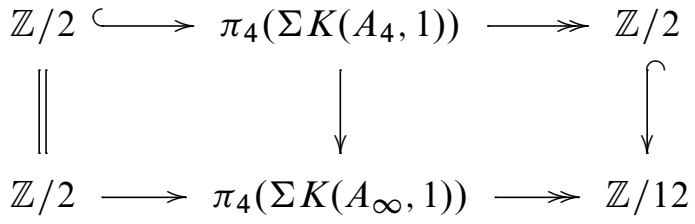

Now we use the isomorphism (5-2). Consider the suspension

$$
K\left(A_{\infty}, 1\right)^{+} \rightarrow \Omega \Sigma K\left(A_{\infty}, 1\right)^{+} \simeq \Omega \Sigma K\left(A_{\infty}, 1\right)
$$

and the corresponding map between Whitehead sequences:

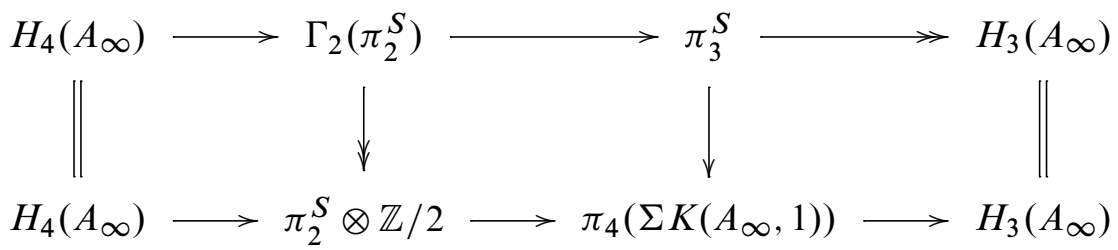

Since

$$
\pi_{3}^{S}=\mathbb{Z} / 24, \quad \pi_{4}^{S}=0,
$$

we conclude that the Whitehead sequence for $K\left(A_{\infty}, 1\right)^{+}$has the following form:

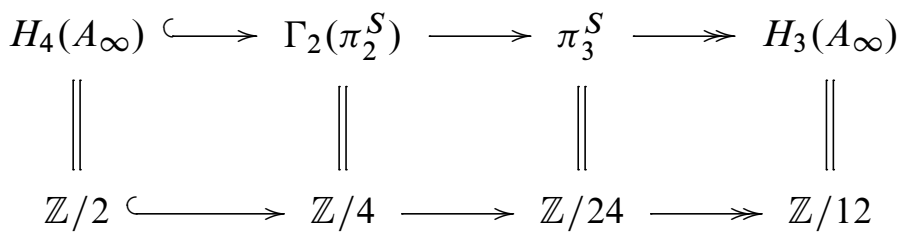

We conclude that the map

$$
\pi_{3}^{S} \rightarrow \pi_{4}\left(\Sigma K\left(A_{\infty}, 1\right)\right)
$$


is an isomorphism and that the map

$$
H_{4}\left(A_{\infty}\right) \rightarrow \Gamma_{2}^{2}(0 \rightarrow \mathbb{Z} / 6)
$$

is the zero map. The diagram (5-7) has the following form:

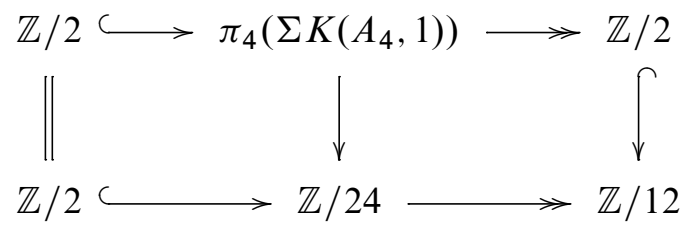

The result follows.

Acknowledgements The research of the first author is partially supported by RFBR (grant 08-01-91300 $\mathrm{IND}_{a}$ ) and RF Presidential grant MK-3644.2009.01. The research of the second author is partially supported by the Academic Research Fund of the National University of Singapore R-146-000-101-112.

\section{References}

[1] D Arlettaz, Algebraic $K$-theory of rings from a topological viewpoint, Publ. Mat. 44 (2000) 3-84 MR1775748

[2] H-J Baues, Homotopy type and homology, Oxford Math. Monogr., Oxford Science Publ., The Clarendon Press, Oxford Univ. Press, New York (1996) MR1404516

[3] H J Baues, D Conduché, On the tensor algebra of a nonabelian group and applications, $K$-Theory 5 (1991/92) 531-554 MR1169422

[4] H-J Baues, P Goerss, A homotopy operation spectral sequence for the computation of homotopy groups, Topology 39 (2000) 161-192 MR1710998

[5] L Breen, On the functorial homology of abelian groups, J. Pure Appl. Algebra 142 (1999) 199-237 MR1721092

[6] R Brown, J-L Loday, Van Kampen theorems for diagrams of spaces, Topology 26 (1987) 311-335 MR899052 With an appendix by M Zisman

[7] G Carlsson, A simplicial group construction for balanced products, Topology 23 (1984) 85-89 MR721454

[8] A Dold, D Puppe, Homologie nicht-additiver Funktoren. Anwendungen, Ann. Inst. Fourier Grenoble 11 (1961) 201-312 MR0150183

[9] S Eilenberg, S Mac Lane, On the groups $H(\Pi, n)$. II. Methods of computation, Ann. of Math. (2) 60 (1954) 49-139 MR0065162 
[10] G Ellis, R Mikhailov, A colimit of classifying spaces, to appear Adv. Math. arXiv: 0804.3581

[11] J C Harris, N J Kuhn, Stable decompositions of classifying spaces of finite abelian p-groups, Math. Proc. Cambridge Philos. Soc. 103 (1988) 427-449 MR932667

[12] J Neisendorfer, Primary homotopy theory, Mem. Amer. Math. Soc. 25 (1980) iv+67 MR567801

[13] S B Priddy, On $\Omega^{\infty} S^{\infty}$ and the infinite symmetric group, from: "Algebraic topology (Proc. Sympos. Pure Math., Vol. XXII, Univ. Wisconsin, Madison, Wis., 1970)”, (A Liulevicius, editor), Amer. Math. Soc. (1971) 217-220 MR0358767

[14] D G Quillen, Spectral sequences of a double semi-simplicial group, Topology 5 (1966) 155-157 MR0195097

[15] H Toda, Composition methods in homotopy groups of spheres, Annals of Math. Studies 49, Princeton Univ. Press (1962) MR0143217

[16] H Toda, Order of the identity class of a suspension space, Ann. of Math. (2) 78 (1963) 300-325 MR0156347

[17] G W Whitehead, On spaces with vanishing low-dimensional homotopy groups, Proc. Nat. Acad. Sci. U. S. A. 34 (1948) 207-211 MR0028027

[18] J H C Whitehead, The homotopy type of a special kind of polyhedron, Ann. Soc. Polon. Math. 21 (1948) 176-186 (1949) MR0030758

[19] J H C Whitehead, A certain exact sequence, Ann. of Math. (2) 52 (1950) 51-110 MR0035997

[20] J Wu, Combinatorial descriptions of homotopy groups of certain spaces, Math. Proc. Cambridge Philos. Soc. 130 (2001) 489-513 MR1816806

[21] J Wu, Homotopy theory of the suspensions of the projective plane, Mem. Amer. Math. Soc. 162 (2003) x+130 MR1955357

Steklov Mathematical Institute

Gubkina 8, Moscow 119991, Russia

Department of Mathematics, National University of Singapore

2Block S17 (SOC1), 06-02, 10 Lower Kent Ridge Road, Singapore 119076, Singapore

romanvm@mi.ras.ru, matwuj@nus.edu.sg

www.math.nus.edu.sg/ matwujie

Received: 26 October 2009 